\title{
THE WEIL ALGEBRA AND THE SECONDARY CHARACTERISTIC HOMOMORPHISM OF REGULAR LIE ALGEBROIDS
}

\author{
JAN KUBARSKI \\ Institute of Mathematics, Technical University of Eódź \\ al. Politechniki 11, 90-924 Eódź, Poland \\ E-mail:kubarski@ck-sg.p.lodz.pl \\ and \\ Institute of Mathematics and Informatics \\ Częstochowa Technical University \\ Dabrowskiego 69, 42-201 Częstochowa, Poland
}

\begin{abstract}
This work gives a construction of the secondary characteristic homomorphism in the category of regular Lie algebroids generalizing the theory of Kamber-Tondeur for foliated principal bundles equipped with reductions. Part I is a preparation and concerns the concept of the Weil algebra $\mathcal{W} \boldsymbol{g}$ of the Lie algebra bundle $\boldsymbol{g}$ adjoint to a regular Lie algebroid $A$. A fundamental role is played by its subalgebra $(\mathcal{W} \boldsymbol{g})_{I^{0}}$ of invariant cross-sections with respect to adjoint representations. In Part II we give a construction of characteristic invariants of partially flat regular Lie algebroids, measuring the incompatibility of two geometric structures: a partially flat connection and a Lie subalgebroid. This generalizes the classical construction of KamberTondeur. Fundamental properties, for example, independence of the homotopy class of a Lie subalgebroid, are given.

A comparison of the presented Lie algebroid theory with characteristic classes of foliated principal bundles shows the algebroid nature of the latter. For globally flat connnections the concept reduces to characteristic invariants of flat regular Lie algebroids in [K7].

In the Appendix we present the elementary theory of regular Lie algebroids in which the key role is played by a global theorem on solutions of some system of partial differential equations with parameters. One of the main structure theorems concerns invariant cross-sections on $\mathbb{R} \times M$, the basic fact needed in the proof of homotopy independence of characteristic classes.
\end{abstract}

2000 Mathematics Subject Classification: Primary 57R20; Secondary 53C05, 53D17.

Key words and phrases: Lie algebroid, connection, flat connection, Chern-Weil homomorphism, secondary characteristic classes, Weil algebra, truncated Weil algebra, Poisson manifold.

The paper is in final form and no version of it will be published elsewhere. 
1. Introduction. This work belongs to the direction initiated by K. Mackenzie in [Ma1, Chap. III, IV] and by Ph. Higgins \& K. Mackenzie in [H-M] and developed by the author ([K2], [K6], [K8], [K9], [K10]) and others and concerns the "pure" theory of Lie algebroids. These works isolate this theory from the common theory of Lie groupoids and Lie algebroids.

Originally, Lie algebroids were invented by J. Pradines (1967) in connection with the study of differential groupoids, generalizing the construction of the Lie algebra of a Lie group. Since every principal bundle $P$ determines a Lie groupoid $P P^{-1}$ of Ehresmann [E], therefore - in an indirect manner - it determines a Lie algebroid $A(P)$. The construction of this object with the omission of the step of Lie groupoids (with the use of the vector bundle $T P / G$ considered by $\mathrm{M}$. Atiyah [At] and P. Libermann [L]) was made independently by K. Mackenzie [Ma1] and by the author [K1]. In [K1] there is also a third manner of constructing a Lie algebroid of a principal bundle $P(M, G)$ as an associated bundle $W^{1}(P) \times_{G_{n}^{1}}\left(\mathbb{R}^{n} \times \mathfrak{g}\right)$ with the first-order prolongation of $P$.

Since 1977 another source of transitive Lie algebroids has been known, namely, the theory of transversally complete foliations [Mo1], in particular, the theory of foliations of left cosets of nonclosed Lie subgroups in Lie groups. On this ground R. Almeida and P. Molino discovered in 1985 [A-M] (see also [Mo2]) non-integrable transitive Lie algebroids. In [K3] the author gives a direct definition of the Lie algebroid of the TCfoliation of left cosets without using Molino's theory.

Differential geometry revealed also other objects which yield Lie algebroids: Poisson manifolds [C-D-W], some complete closed pseudogroups [S] and Jacobi manifolds [K-SB]. The results of pure theory of Lie algebroids can be used just to these geometric categories which are a potential source of new results and methods.

Problem 1.1. Can the Chern-Weil characteristic classes on the ground of principal bundles be constructed on the level of Lie algebroids?

This problem was considered first by N. Teleman in 1972 [T1], [T2]. He constructed the classes for some exact extensions of $R$-Lie-Rinehart algebras [under the terminology of J. Huebschmann [H1]], $R$ being a commutative unital ring containing rational numbers $\mathbb{Q} \subset R$. He also noticed that his construction is a generalization of the classical bundle case, provided that the structure Lie groups are connected. The same result was repeated in [K1] by keeping the apparatus of principal bundles only. The full answer is included in [K2] and independently repeated by I. Belko [B1], [B2]. In [K2] one can find a construction of an equivalent of the Chern-Weil homomorphism for regular Lie algebroids over foliated manifolds whereas in [B1], [B2] for transitive case only. For application to the tangential case (strengthening the Moore-Schochet construction [M-Sch]) see [K5]. In [K2], [B1] and [B2] there is an observation that the Chern-Weil homomorphism of a connected principal bundle is an invariant of the Lie algebroid of this bundle (the structure Lie group may be disconnected). In [K2] a class of transitive non-integral Lie algebroids having nontrivial Chern-Weil homomorphism is discovered (on the ground of TC-foliations of left cosets of nonclosed Lie groups in Lie groups). J. Huebschmann [H2] generalized results of [T2] and [K2] on the exact extensions of $R$-Lie Rinehart algebras without the assumption $\mathbb{Q} \subset R$. We add that Lie-Rinehart algebras are algebraic equivalents 
of Lie algebroids and were known - under different names - considerably earlier than Lie algebroids (namely, since 1953 (Herz), see the survey article by K. Mackenzie [Ma2]). The Chern-Weil homomorphism can be generalized to pairs of Lie algebroids $(L, A)$ with the use of the so-called $L$-connections in $A$ understood as linear homomorphisms $\nabla: L \rightarrow A$ compatible with anchors, $\gamma_{A} \circ \nabla=\gamma_{L}$, [B-K-W]. The case when $A=T P / G$ for a principal fibre bundle $P(M, G)$ or $A=A(\mathfrak{f})$ for a vector bundle $\mathfrak{f}$ was considered independently by R. L. Fernandes [F2] and M. Crainic [Cr].

The extension of Problem 1.1 also for secondary characteristic classes of flat or partially flat principal bundles was posed by the author in the late eighties. The full answer concerning characteristic classes of flat or partially flat objects was accomplished (in manuscript) in 1989, submitted in 1992 to Publ. Départ. Math., Université Claude Bernard - Lyon 1, and accepted (see [D, 1994]). However, this work has not appeared in print because the publication of the journal was discontinued (see http://www.desargues. univ-lyon1.fr/home/portier/bib.html). For a sketch of these results see [K4]. Part of results concerning flat objects appeared in [K7] in 1998.

The present work (Part I and Part II) is devoted to characteristic classes of partially flat regular Lie algebroids constructed in the spirit developed by Kamber and Tondeur of foliated bundles equipped with a reduction [K-T1], [K-T2]. In part I the Weil algebra for the Lie algebra bundle adjoint to a regular Lie algebroid is constructed (adapting classical theory for Lie algebras [Ca], [K-T2]); some ideas of G. Andrzejczak (unpublished) of a change of variables in the Weil algebra are used. As an explicit application, the Chern-Weil homomorphism of regular Lie algebroids is obtained once more. The results of part I are done as a preparation for construction of the secondary characteristic homomorphism, the aim of part II, measuring the incompatibility of two geometric structures on a regular Lie algebroid: a partially flat connection and a given Lie subalgebroid. The tangent bundle to a characteristic foliation of a regular Lie algebroid considered plays the role of the tangent bundle for classical characteristic classes. The case of transitive Lie algebroids $B \subset A$ and a partially flat connection over a regular foliation $\mathcal{F}$ is a simple generalization of the characteristic homomorphism for a regular foliation (for $A$ the Lie algebroid of the principal bundle $P$ of transversal frames and $B$ the Lie algebroid of some $O(n)$ reduction in $P$ ).

Recently, Rui Loja Fernandes [F2] proposes an approach to secondary characteristic classes including singular Stefan's foliations appearing as the images of anchors in Lie algebroids. The Fernandes approach is in the spirit of the original papers of Chern and Simons [C-S1], [C-S2] of comparing two connections: a basic connection and a metric one acting from a given (nonregular in general) Lie algebroid $A$. This is very important in Poisson geometry [F1]. M. Crainic [Cr] has defined secondary characteristic classes for a representation of a Lie algebroid, i.e. a Lie algebroid homomorphism $L \rightarrow A(\mathfrak{f})$. They vanish if the values of the representation are contained in the Lie subalgebroid of some $O(n)$-reduction of $\mathfrak{f}$. For a general approach to Lie algebroids of $G$-vector bundles see $[\mathrm{K} 5]$. The common part of the Crainic theory and the one considered in this paper concerns Lie algebroids $L=F \subset T M$, and partial connections in vector bundles $\mathfrak{f}$, $\lambda^{\prime}: L=F \rightarrow A=A(\mathfrak{f})$ and Lie subalgebroids $B \subset A$ which are $O(n)$-reductions of $\mathfrak{f}$. In my opinion, it is possible to unify the Fernandes and Crainic theory with the one below. 


\section{PART I. WEIL ALGEBRA FOR REGULAR LIE ALGEBROIDS}

2. Homomorphism $(d \omega)^{\vee}$. We continue our work [K2], [K7] concerning characteristic homomorphisms of regular Lie algebroids. We assume that all the manifolds considered are $C^{\infty}$ and Hausdorff, and that the manifolds $M, M^{\prime} \ldots$ over which Lie algebroids are considered, are, in addition, connected. By $\Omega^{0}(M)$ we denote the ring of $C^{\infty}$ functions on a manifold $M$, by $\mathfrak{X}(M)$ the Lie algebra of $C^{\infty}$ vector fields on $M$, and by $\operatorname{Sec} A$ the $\Omega^{0}(M)$-module of all $C^{\infty}$ global cross-sections of a given vector bundle $A$ over $M$.

By a regular Lie algebroid over a foliated manifold $(M, E)$, we mean a system $A=$ $(A, \llbracket \cdot, \cdot \rrbracket, \gamma)$ consisting of a vector bundle $A$ on $M$ and mappings $\llbracket \cdot, \cdot \rrbracket: \operatorname{Sec} A \times \operatorname{Sec} A \rightarrow$ $\operatorname{Sec} A, \gamma: A \rightarrow T M$, such that (1) (Sec $A, \llbracket \cdot, \cdot \rrbracket)$ is an $\mathbb{R}$-Lie algebra, (2) $\gamma$, called the anchor, is a homomorphism of vector bundles such that $\operatorname{Im} \gamma=E,(3) \llbracket \xi, f \cdot \eta \rrbracket=$ $f \cdot \llbracket \xi, \eta \rrbracket+(\gamma \circ \xi)(f), f \in \Omega^{0}(M)$. The anchor is bracket preserving, i.e. $\operatorname{Sec} \gamma: \operatorname{Sec} A \rightarrow$ $\mathfrak{X}(M), \xi \mapsto \gamma \circ \xi$, is a homomorphism of Lie algebras (see $[\mathrm{H}],[\mathrm{B}-\mathrm{K}-\mathrm{W}]$ ). In the sequel, we adopt the notions and the notations from [Ma1], [K2] and mainly from [K7]. We also write $\bigwedge W$ and $\bigvee W$ for the exterior and symmetric algebras for a given vector space $W$.

Let $A=(A, \llbracket \cdot, \cdot \rrbracket, \gamma)$ be an arbitrary regular Lie algebroid over a foliated manifold $(M, E)$ with the Atiyah sequence $0 \rightarrow \boldsymbol{g} \hookrightarrow A \stackrel{\gamma}{\rightarrow} E \rightarrow 0$, equipped with a connection $\lambda: E \rightarrow A$ having $\omega: A \rightarrow \boldsymbol{g}$ as its connection form. Below, the exterior derivative of forms on the Lie algebroid $A$, with values in $\boldsymbol{g}$, [also in the associated vector bundles] with respect to the adjoint representation $\operatorname{ad}_{A}: A \rightarrow A(\boldsymbol{g})$ [or induced ones] will be briefly denoted by $d^{g}$. $d^{g} \omega$ at a point $x \in M$ is a 2-linear skew-symmetric tensor $\left(d^{g} \omega\right)_{\mid x}$ : $A_{\mid x} \times A_{\mid x} \rightarrow \boldsymbol{g}_{\mid x}$ understood sometimes equivalently as an element of $\bigwedge^{2} A_{\mid x}^{*} \otimes \boldsymbol{g}_{\mid x}$. It defines a linear mapping with the property

$$
(d \omega)_{x}\left(w_{1}^{*}\right) \wedge(d \omega)_{x}\left(w_{2}^{*}\right)=(d \omega)_{x}\left(w_{2}^{*}\right) \wedge(d \omega)_{x}\left(w_{1}^{*}\right), \quad w_{i}^{*} \in \boldsymbol{g}_{\mid x}^{*} .
$$

Therefore, by the universal property of the symmetric algebra $\bigvee \boldsymbol{g}_{\mid x}^{*}$, see [G], we obtain the existence and uniqueness of a homomorphism of algebras

$$
(d \omega)_{x}^{\vee}: \bigvee g_{\mid x}^{*} \rightarrow \bigwedge A_{\mid x}^{*}
$$

extending $(d \omega)_{x}$ and such that $(d \omega)_{x}^{\vee}(1)=1$.

Lemma 2.1. Let $\Gamma \in \bigvee^{l} \boldsymbol{g}_{\mid x}^{*}$, then for $w_{1}, \ldots, w_{2 l} \in A_{\mid x}$

$$
\begin{aligned}
& \left\langle(d \omega)_{x}^{\vee}(\Gamma), w_{1} \wedge \ldots \wedge w_{2 l}\right\rangle \\
& =\frac{1}{l ! \cdot 2^{l}} \sum_{\sigma} \operatorname{sgn} \sigma \cdot\left\langle\Gamma,\left(d^{g} \omega\right)_{\mid x}\left(w_{\sigma_{1}} \wedge w_{\sigma_{2}}\right) \vee \ldots \vee\left(d^{g} \omega\right)_{\mid x}\left(w_{\sigma_{2 l-1}} \wedge w_{\sigma_{2 l}}\right)\right\rangle .
\end{aligned}
$$

Proof. It is sufficient to prove this for a simple tensor $\Gamma=w_{1}^{*} \vee \ldots \vee w_{l}^{*}$ :

$$
\begin{aligned}
& \left\langle(d \omega)_{x}^{\vee}\left(w_{1}^{*} \vee \ldots \vee w_{l}^{*}\right), w_{1} \wedge \ldots \wedge w_{2 l}\right\rangle \\
& =\left\langle(d \omega)_{x}\left(w_{1}^{*}\right) \wedge \ldots \wedge(d \omega)_{x}\left(w_{l}^{*}\right), w_{1} \wedge \ldots \wedge w_{2 l}\right\rangle \\
& =\frac{1}{2^{l}} \sum_{\sigma} \operatorname{sgn} \sigma \cdot(d \omega)_{x}\left(w_{1}^{*}\right)\left(w_{\sigma_{1}} \wedge w_{\sigma_{2}}\right) \cdot \ldots \cdot(d \omega)_{x}\left(w_{l}^{*}\right)\left(w_{\sigma_{2 l-1}} \wedge w_{\sigma_{2 l}}\right) \\
& =\frac{1}{2^{l}} \sum_{\sigma} \operatorname{sgn} \sigma \cdot\left\langle w_{1}^{*},\left(d^{g} \omega\right)_{\mid x}\left(w_{\sigma_{1}} \wedge w_{\sigma_{2}}\right)\right\rangle \cdot \ldots \cdot\left\langle w_{l}^{*},\left(d^{g} \omega\right)_{\mid x}\left(w_{\sigma_{2 l-1}} \wedge w_{\sigma_{2 l}}\right)\right\rangle
\end{aligned}
$$




$$
\begin{gathered}
=\sum_{\substack{\sigma_{1}<\sigma_{2}, \ldots, \sigma_{2 l-1}<\sigma_{2 l} \\
\sigma_{1}<\sigma_{3}<\ldots<\sigma_{2 l-1}}} \operatorname{sgn} \sigma \cdot \sum_{\tau}\left\langle w_{1}^{*},\left(d^{g} \omega\right)_{\mid x}\left(w_{\sigma_{2 \cdot \tau}(1)-1} \wedge w_{\sigma_{2 \cdot \tau}(1)}\right)\right\rangle \cdot \ldots \\
\cdots \cdot\left\langle w_{l}^{*},\left(d^{g} \omega\right)_{\mid x}\left(w_{\sigma_{2 \cdot \tau(l)-1}} \wedge w_{\sigma_{2 \cdot \tau(l)}}\right)\right\rangle \\
=\frac{1}{l ! \cdot 2^{l}} \sum_{\sigma} \operatorname{sgn} \sigma \cdot\left\langle w_{1}^{*} \vee \ldots \vee w_{l}^{*},\left(d^{g} \omega\right)_{\mid x}\left(w_{\sigma_{1}} \wedge w_{\sigma_{2}}\right) \vee \ldots\right. \\
\left.\vee\left(d^{g} \omega\right)_{\mid x}\left(w_{\sigma_{2 l-1}} \wedge w_{\sigma_{2 l}}\right)\right\rangle .
\end{gathered}
$$

According to this lemma and the fact that the canonical duality $\bigvee^{l} \boldsymbol{g}^{*} \times \bigvee^{l} \boldsymbol{g} \rightarrow \mathbb{R}$ [defined pointwise by the permanent] is a $C^{\infty}$ 2-linear homomorphism of vector bundles, we have the following

COROllary 2.2. For $\Gamma \in \bigvee^{l} \boldsymbol{g}^{*}$, the cross-section

$$
(d \omega)^{\vee}(\Gamma): M \rightarrow \bigwedge^{2 l} A^{*}, x \mapsto(d \omega)_{x}^{\vee}\left(\Gamma_{x}\right),
$$

is a $C^{\infty}$ real $2 l$-form on $A$, i.e. $(d \omega)^{\vee}(\Gamma) \in \Omega_{A}^{2 l}(M)$, and it is defined by

$$
(d \omega)^{\vee}(\Gamma)=\frac{1}{l !} \cdot\left\langle\Gamma, d^{g} \omega \vee \ldots \vee d^{g} \omega\right\rangle
$$

3. Weil algebra of the bundle $\boldsymbol{g}$ of Lie algebras. For the bundle $\boldsymbol{g}$ of Lie algebras, adjoint to a given regular Lie algebroid $A$, we have:

- $\bigwedge \boldsymbol{g}_{\mid x}^{*}$ is an anticommutative graded algebra; $\left(\bigwedge \boldsymbol{g}_{\mid x}^{*}\right)^{k}:=\bigwedge^{k} \boldsymbol{g}_{\mid x}^{*}, x \in M$,

- $\bigvee \boldsymbol{g}_{\mid x}^{*}$ is an (anti)commutative graded algebra over the graded vector space $\boldsymbol{g}_{\mid x}^{*}$ with elements of degree two only, i.e. $\left(\bigvee \boldsymbol{g}_{\mid x}^{*}\right)^{2 l}=\bigvee^{l} \boldsymbol{g}_{\mid x}^{*}$ and $\left(\bigvee \boldsymbol{g}_{\mid x}^{*}\right)^{2 l+1}=0$.

- $W \boldsymbol{g}_{\mid x}:=\bigwedge \boldsymbol{g}_{\mid x}^{*} \otimes \bigvee \boldsymbol{g}_{\mid x}^{*}$ is the anticommutative (bi)graded tensor product of anticommutative graded algebras. The bidegree $\left(W \boldsymbol{g}_{\mid x}\right)^{k, 2 l}=\bigwedge^{k} \boldsymbol{g}_{\mid x}^{*} \otimes \bigvee^{l} \boldsymbol{g}_{\mid x}^{*}$ leads, as usual, to the total degree $r=k+2 l$.

$W \boldsymbol{g}_{\mid x}$ as an algebra is generated by $1, w^{*} \otimes 1$ and $1 \otimes w^{*}$, for $w^{*} \in \boldsymbol{g}_{\mid x}^{*}$. Put

$$
\begin{gathered}
(W \boldsymbol{g})^{k, 2 l}:=\bigwedge^{k} \boldsymbol{g}^{*} \otimes \bigvee^{l} \boldsymbol{g}^{*}, \quad(W \boldsymbol{g})^{r}:=\bigoplus_{k+2 l=r}(W \boldsymbol{g})^{k, 2 l}, \\
(\mathcal{W} \boldsymbol{g})^{k, 2 l}:=\operatorname{Sec}(W \boldsymbol{g})^{k, 2 l}, \quad(\mathcal{W} \boldsymbol{g})^{r}:=\operatorname{Sec}(W \boldsymbol{g})^{r}\left(=\bigoplus_{k+2 l=r}(\mathcal{W} \boldsymbol{g})^{k, 2 l}\right), \\
\mathcal{W} \boldsymbol{g}:=\bigoplus_{r}(\mathcal{W} \boldsymbol{g})^{r} .
\end{gathered}
$$

- $\mathcal{W} \boldsymbol{g}$ is a bigraded algebra with the multiplication defined pointwise.

$\mathcal{W} \boldsymbol{g}$ is called the Weil algebra of the bundle $\boldsymbol{g}$ of Lie algebras. Each element of $\mathcal{W} \boldsymbol{g}$ is locally [even globally, by the paracompactness of $M$ ] a finite sum of cross-sections of the form $\psi_{1} \wedge \cdots \wedge \psi_{k} \otimes \Gamma_{1} \vee \cdots \vee \Gamma_{l}, \quad \psi_{i}, \Gamma_{j} \in \operatorname{Sec} \boldsymbol{g}^{*}, k, l \geq 0$. Here (and below), $k, l, r$ are nonnegative integers.

REMARK 3.1. Under the gradation considered, the homomorphism (2.1) is of degree 0 . Analogously, introducing the "pointwise" structure of an algebra in $\bigoplus_{l \geq 0}$ Sec $\bigvee^{l} \boldsymbol{g}^{*}$ 
and the gradation as above, we see that

$$
(d \omega)^{\vee}: \bigoplus_{l \geq 0} \operatorname{Sec} \bigvee^{l} \boldsymbol{g}^{*} \rightarrow \Omega_{A}(M)
$$

is a homomorphism of algebras of degree 0 .

4. Auxiliary operators $\overline{\boldsymbol{k}}, \bar{i}, \bar{d}, \bar{\theta}$. Three fundamental operators $i, d, \theta$ in $\mathcal{W g}$, as well as the mapping $\boldsymbol{k}: \mathcal{W} \boldsymbol{g} \rightarrow \Omega_{A}(M)$, will be introduced in two steps passing through some isomorphisms $\psi_{x}: W \boldsymbol{g}_{\mid x} \rightarrow W \boldsymbol{g}_{\mid x}, x \in M$ [i.e. some change of variables]. This method, due to G. Andrzejczak (unpublished), enables us to define and prove the property of these objects in the clear and technically lucid manner. The main profit is that the differential $d$ is then defined by one simple formula.

We begin by defining some auxiliary objects $\overline{\boldsymbol{k}}, \bar{i}, \bar{d}, \bar{\theta}$. Firstly, we observe that, for each point $x \in M$, there exists exactly one homomorphism

$$
\overline{\boldsymbol{k}}_{x}: W \boldsymbol{g}_{\mid x} \rightarrow \bigwedge A_{\mid x}^{*}
$$

of algebras of degree 0 such that $\overline{\boldsymbol{k}}_{x}(1)=1, \overline{\boldsymbol{k}}_{x}\left(w^{*} \otimes 1\right)=\omega_{x}^{\wedge}\left(w^{*}\right)$ (for $\omega_{x}^{\wedge}$ see [K7]) and $\overline{\boldsymbol{k}}_{x}\left(1 \otimes w^{*}\right)=(d \omega)_{x}^{\vee}\left(w^{*}\right)$ when $w^{*} \in \boldsymbol{g}_{\mid x}^{*} \cdot \overline{\boldsymbol{k}}_{x}$ is directly defined by the formula $\overline{\boldsymbol{k}}_{x}\left(\Psi_{x} \otimes \Gamma_{x}\right)=\omega_{x}^{\wedge}\left(\Psi_{x}\right) \wedge(d \omega)_{x}^{\vee}\left(\Gamma_{x}\right)$ for $\Psi_{x} \in \wedge \boldsymbol{g}_{\mid x}^{*}$ and $\Gamma_{x} \in \bigvee \boldsymbol{g}_{\mid x}^{*}$. The homomorphisms $\overline{\boldsymbol{k}}_{x}, x \in M$, give rise to the homomorphism

$$
\overline{\boldsymbol{k}}: \mathcal{W} \boldsymbol{g} \rightarrow \Omega_{A}(M)
$$

of algebras of degree 0 defined pointwise: $\overline{\boldsymbol{k}}(\Psi \otimes \Gamma)_{x}=\overline{\boldsymbol{k}}_{x}\left(\Psi_{x} \otimes \Gamma_{x}\right), \Psi \in \bigoplus_{k \geq 0} \operatorname{Sec} \bigwedge^{k} \boldsymbol{g}^{*}$, $\Gamma \in \bigoplus_{l \geq 0} \operatorname{Sec} \bigvee^{l} \boldsymbol{g}^{*}$. It has the property

$$
\overline{\boldsymbol{k}}(\Psi \otimes \Gamma)=\omega^{\wedge}(\Psi) \wedge(d \omega)^{\vee}(\Gamma) .
$$

Lemma 4.1. For each $x \in M$ and for $v \in \boldsymbol{g}_{\mid x}$, there exists exactly one antiderivation $\bar{i}_{x, v}: W \boldsymbol{g}_{\mid x} \rightarrow W \boldsymbol{g}_{\mid x}$ of degree -1 such that

(1) $\bar{i}_{x, v}\left(w^{*} \otimes 1\right)=\left\langle w^{*}, v\right\rangle$,

(2) $\bar{i}_{x, v}\left(1 \otimes w^{*}\right)=-\left(w^{*} \circ \operatorname{ad}_{v}\right) \otimes 1, w^{*} \in \boldsymbol{g}_{\mid x}^{*}$.

It has the following properties:

(i) $\bar{i}_{x, v} \mid\left(W \boldsymbol{g}_{\mid x}\right)^{0,0}=0$,

(ii) $\bar{i}_{x, v}\left(1 \otimes w_{1}^{*} \vee \cdots \vee w_{l}^{*}\right)=-\sum_{i} w_{i}^{*} \circ \operatorname{ad}_{v} \otimes w_{1}^{*} \vee \cdots \widehat{i} \cdots \vee w_{l}^{*}, \quad l \geq 1$,

(iii) $\bar{i}_{x, v}\left(\Psi_{x} \otimes \Gamma_{x}\right)=i_{v}\left(\Psi_{x}\right) \otimes \Gamma_{x}+(-1)^{k}\left(\Psi_{x} \otimes 1\right) \cdot \bar{i}_{x, v}\left(1 \otimes \Gamma_{x}\right)$ when $\Psi_{x} \in \bigwedge^{k} \boldsymbol{g}_{\mid x}^{*}$ and $\Gamma_{x} \in \bigvee \boldsymbol{g}_{\mid x}^{*}$,

(iv) $\bar{i}_{x, v}\left[\left(W \boldsymbol{g}_{\mid x}\right)^{k, 2 l}\right] \subset\left(W \boldsymbol{g}_{\mid x}\right)^{k-1,2 l} \oplus\left(W \boldsymbol{g}_{\mid x}\right)^{k+1,2(l-1)}$.

Proof. Uniqueness. The uniqueness of $\bar{i}_{x, v}$ is evident because every antiderivation is uniquely determined by the values on generators. Properties (i)-(iv) of each antiderivation $\bar{i}_{x, v}$ fulfilling (1) and (2) above are evident.

Existence. For $l \geq 1$, there exists exactly one linear mapping

$$
\widetilde{i}_{x, v}^{l}: \bigvee^{l} \boldsymbol{g}_{\mid x}^{*} \rightarrow \boldsymbol{g}_{\mid x}^{*} \otimes \bigvee^{l-1} \boldsymbol{g}_{\mid x}^{*}
$$


such that $\widetilde{i}_{x, v}^{l}\left(w_{1}^{*} \vee \cdots \vee w_{l}^{*}\right)=-\sum_{i} w_{i}^{*} \circ \operatorname{ad}_{v} \otimes w_{1}^{*} \vee \cdots \widehat{i} \cdots \vee w_{l}^{*}$. It has the property

$$
\widetilde{i}_{x, v}^{m+n}\left(\Gamma_{1 x} \vee \Gamma_{2 x}\right)=\widetilde{i}_{x, v}^{m}\left(\Gamma_{1 x}\right) \cdot 1 \otimes \Gamma_{2 x}+1 \otimes \Gamma_{1 x} \cdot \widetilde{i}_{x, v}^{n}\left(\Gamma_{2 x}\right)
$$

for $\Gamma_{1 x} \in \bigvee^{m} \boldsymbol{g}_{\mid x}^{*}$ and $\Gamma_{2 x} \in \bigvee^{n} \boldsymbol{g}_{\mid x}^{*}$. For $k \geq 0$ and $l \geq 1$, there exists exactly one linear mapping

$$
\bar{i}_{x, v}^{k, 2 l}:\left(W \boldsymbol{g}_{\mid x}\right)^{k, 2 l} \rightarrow\left(W \boldsymbol{g}_{\mid x}\right)^{k-1,2 l} \oplus\left(W \boldsymbol{g}_{\mid x}\right)^{k+1,2(l-1)}
$$

such that $\bar{i}_{x, v}^{k, 2 l}\left(\Psi_{x} \otimes \Gamma_{x}\right)=i_{v}\left(\Psi_{x}\right) \otimes \Gamma_{x}+(-1)^{k}\left(\Psi_{x} \otimes 1\right) \cdot \widetilde{i}_{x, v}^{l}\left(\Gamma_{x}\right)$ when $\Psi_{x} \in \bigwedge^{k} \boldsymbol{g}_{\mid x}^{*}$ and $\Gamma_{x} \in \bigvee \boldsymbol{g}_{\mid x}^{*}$. Set, additionally, $\vec{i}_{x, v}^{-, 0}=0$ and

$$
\bar{i}_{x, v}^{k, 0}:\left(W \boldsymbol{g}_{\mid x}\right)^{k, 0} \rightarrow\left(W \boldsymbol{g}_{\mid x}\right)^{k-1,0}, \Psi_{x} \otimes 1 \mapsto i_{v} \Psi_{x} \otimes 1,
$$

for $k \geq 1$. All the linear mappings $\bar{i}_{x, v}^{k, 2 l}, k, l \geq 0$, together define the operator

$$
\bar{i}_{x, v}=\sum_{k, l \geq 0} \bar{i}_{x, v}^{k, 2 l}: W \boldsymbol{g}_{\mid x} \rightarrow W \boldsymbol{g}_{\mid x} .
$$

Of course, $\bar{i}_{x, v}$ satisfies (1) and (2). It remains to show that $\bar{i}_{x, v}$ is an antiderivation of degree -1 , i.e. $\bar{i}_{x, v}\left(\Theta_{1} \cdot \Theta_{2}\right)=\bar{i}_{x, v}\left(\Theta_{1}\right) \cdot \Theta_{2}+(-1)^{r} \Theta_{1} \cdot \bar{i}_{x, v}\left(\Theta_{2}\right)$ for $\Theta_{1} \in\left(W \boldsymbol{g}_{\mid x}\right)^{r}$, $\Theta_{2} \in W \boldsymbol{g}_{\mid x}$, which is easy to obtain by considering elements $\Theta_{i}$ homogeneous with respect to the bigradation only.

For a cross-section $v \in \operatorname{Sec} \boldsymbol{g}$ and for $\Theta \in \mathcal{W} \boldsymbol{g}$, the formula $M \ni x \mapsto \bar{i}_{x, v_{x}}\left(\Theta_{x}\right)$ defines an element $\bar{i}_{v}(\Theta)$ of $\mathcal{W} \boldsymbol{g}$ and

$$
\bar{i}_{v}: \mathcal{W} \boldsymbol{g} \rightarrow \mathcal{W} \boldsymbol{g}, \quad \Theta \mapsto \bar{i}_{v}(\Theta),
$$

is an antiderivation of degree -1 . The smoothness of $\bar{i}_{v}(\Theta)$, by Lemma 4.1(i)-(iii), follows from the smoothness in the cases $\Theta=\Psi \otimes 1$ where $\Psi \in \operatorname{Sec} \bigwedge^{k} \boldsymbol{g}^{*}$, and $\Theta=1 \otimes \Gamma$ where $\Gamma \in \operatorname{Sec} \bigvee^{l} \boldsymbol{g}^{*}$, which is easy to investigate. $\bar{i}_{v}$ has the property

$$
\bar{i}_{v}(\Psi \otimes \Gamma)=i_{v} \Psi \otimes \Gamma+(-1)^{k}(\Psi \otimes 1) \cdot \bar{i}_{v}(1 \otimes \Gamma)
$$

for $\Psi \in \operatorname{Sec} \bigwedge^{k} \boldsymbol{g}^{*}$ and $\Gamma \in \operatorname{Sec} \bigvee^{l} \boldsymbol{g}^{*}$.

LEMma 4.2. For each $x \in M$, there exists exactly one antiderivation $\bar{d}_{x}: W \boldsymbol{g}_{\mid x} \rightarrow$ $W \boldsymbol{g}_{\mid x}$ of degree +1 such that

1. $\bar{d}_{x}\left(w^{*} \otimes 1\right)=1 \otimes w^{*}$,

2. $\bar{d}_{x}\left(1 \otimes w^{*}\right)=0, w^{*} \in \boldsymbol{g}_{\mid x}^{*}$.

It has the properties:

(i) $\bar{d}_{x} \mid\left(W \boldsymbol{g}_{\mid x}\right)^{0,0}=0$,

(ii) $\bar{d}_{x}\left(w_{1}^{*} \wedge \cdots \wedge w_{k}^{*} \otimes 1\right)=\sum_{i}(-1)^{i+1} w_{1}^{*} \wedge \cdots \widehat{i} \cdots \wedge w_{k}^{*} \otimes w_{i}^{*}, \quad k \geq 1$,

(iii) $\bar{d}_{x}\left(\Psi_{x} \otimes \Gamma_{x}\right)=\bar{d}_{x}\left(\Psi_{x} \otimes 1\right) \cdot\left(1 \otimes \Gamma_{x}\right)$ when $\Psi_{x} \in \wedge \boldsymbol{g}_{\mid x}^{*}$ and $\Gamma_{x} \in \bigvee \boldsymbol{g}_{\mid x}^{*}$, in particular, $\bar{d}_{x}\left(1 \otimes \Gamma_{x}\right)=0$

(iv) $\bar{d}_{x}\left[\left(W \boldsymbol{g}_{\mid x}\right)^{k, 2 l}\right] \subset\left(W \boldsymbol{g}_{\mid x}\right)^{k-1,2(l+1)}$,

(v) $\bar{d}_{x}$ is a differential, i.e. $\bar{d}_{x} \circ \bar{d}_{x}=0$.

Proof. Uniqueness. The uniqueness of $\bar{d}_{x}$ and properties (i)-(v) are evident.

Existence. For $k \geq 1$, there exists exactly one linear mapping $\widetilde{d}_{x}^{k}: \bigwedge^{k} \boldsymbol{g}_{\mid x}^{*} \rightarrow$ $\left(\bigwedge^{k-1} \boldsymbol{g}_{\mid x}^{*}\right) \otimes \boldsymbol{g}_{\mid x}^{*}$ such that $\widetilde{d}_{x}^{k}\left(w_{1}^{*} \wedge \cdots \wedge w_{k}^{*}\right)=\sum_{i}(-1)^{i+1} w_{1}^{*} \wedge \cdots \widehat{i} \cdots \wedge w_{k}^{*} \otimes w_{i}^{*}$. It 
has the property $\tilde{d}_{x}^{m+n}\left(\Psi_{1 x} \wedge \Psi_{2 x}\right)=\left(\widetilde{d}_{x}^{m} \Psi_{1 x}\right) \cdot \Psi_{2 x} \otimes 1+(-1)^{m} \Psi_{1 x} \otimes 1 \cdot \widetilde{d}_{x}^{n} \Psi_{2 x}$ when $\Psi_{1 x} \in \bigwedge^{m} \boldsymbol{g}_{\mid x}^{*}$ and $\Psi_{2 x} \in \bigwedge^{n} \boldsymbol{g}_{\mid x}^{*}$. For $k \geq 0$ and $l \geq 1$, there exists exactly one linear mapping $\bar{d}_{x}^{k, 2 l}:\left(W \boldsymbol{g}_{\mid x}\right)^{k, 2 l} \rightarrow\left(W \boldsymbol{g}_{\mid x}\right)^{k-1,2(l+1)}$ such that $\bar{d}_{x}^{k, 2 l}\left(\Psi_{x} \otimes \Gamma_{x}\right)=\left(\widetilde{d}_{x}^{k} \Psi_{x}\right) \cdot\left(1 \otimes \Gamma_{x}\right)$ for $\Psi_{x} \in \bigwedge^{k} \boldsymbol{g}_{\mid x}^{*}$ and $\Gamma_{x} \in \bigvee^{l} \boldsymbol{g}_{\mid x}^{*}$. Add $\bar{d}_{x}^{0,0}=0$ and put $\bar{d}_{x}=\sum_{k, l \geq 0} \bar{d}_{x}^{k, 2 l}: W \boldsymbol{g}_{\mid x} \rightarrow W \boldsymbol{g}_{\mid x}$. Of course, $\bar{d}_{x}$ satisfies (1) and (2). It remains to show that $\bar{d}_{x}$ is an antiderivation of degree +1 which is easy to obtain by considering elements homogeneous with respect to the bigradation.

All homomorphisms $\bar{d}_{x}, x \in M$, define pointwise a homomorphism

$$
\bar{d}: \mathcal{W} \boldsymbol{g} \rightarrow \mathcal{W} \boldsymbol{g}
$$

which is an antiderivation of degree +1 and a differential. It has the property

$$
\bar{d}(\Psi \otimes \Gamma)=\bar{d}(\Psi \otimes 1) \cdot(1 \otimes \Gamma), \quad \Psi \in \operatorname{Sec} \bigwedge^{k} \boldsymbol{g}^{*}, \quad \Gamma \in \operatorname{Sec} \bigvee^{l} \boldsymbol{g}^{*}
$$

LEMma 4.3. For each $x \in M$ and for $v \in \boldsymbol{g}_{\mid x}$, there exists exactly one derivation $\bar{\theta}_{x, v}: W \boldsymbol{g}_{\mid x} \rightarrow W \boldsymbol{g}_{\mid x}$ of degree 0 such that

1. $\bar{\theta}_{x, v}\left(w^{*} \otimes 1\right)=-w^{*} \circ \operatorname{ad}_{v} \otimes 1$,

2. $\bar{\theta}_{x, v}\left(1 \otimes w^{*}\right)=1 \otimes\left(-w^{*} \circ \operatorname{ad}_{v}\right)$.

It has the property:

(i) $\bar{\theta}_{x, v}\left(\Psi_{x} \otimes \Gamma_{x}\right)=\left(\theta_{x}^{\wedge} \Psi_{x}\right) \otimes \Gamma_{x}+\Psi_{x} \otimes\left(\theta_{x}^{\vee} \Gamma_{x}\right)$ when $\Psi_{x} \in \wedge \boldsymbol{g}_{\mid x}^{*}$ and $\Gamma_{x} \in \bigvee \boldsymbol{g}_{\mid x}^{*}$, where $\theta_{x}^{\wedge}$ and $\theta_{x}^{\vee}$ denote the only derivations in the algebras $\wedge \boldsymbol{g}_{\mid x}^{*}$ and $\bigvee \boldsymbol{g}_{\mid x}^{*}$, respectively, induced by $-\operatorname{ad}_{v}^{*}: \boldsymbol{g}_{\mid x}^{*} \rightarrow \boldsymbol{g}_{\mid x}^{*}$.

Proof. The uniqueness and property (i) are evident. Formula (i) gives the required operator.

For $v \in \operatorname{Sec} \boldsymbol{g}$ and $\Theta \in \mathcal{W} \boldsymbol{g}$, the formula $M \ni x \mapsto \bar{\theta}_{x, v_{x}}\left(\Theta_{x}\right)$ defines an element of $\mathcal{W g}$ and

$$
\bar{\theta}_{v}: \mathcal{W} \boldsymbol{g} \rightarrow \mathcal{W} \boldsymbol{g}, \quad \Theta \mapsto \bar{\theta}_{v}(\Theta),
$$

is a derivation of degree 0 . The adjoint representation $\operatorname{ad}_{A}: A \rightarrow A(\boldsymbol{g})[\mathrm{K} 2]$ determines a representation of $A$ on each associated vector bundle such as $\bigwedge^{k} \boldsymbol{g}, \bigvee^{l} \boldsymbol{g}, \bigwedge^{k} \boldsymbol{g}^{*} \otimes \bigvee^{l} \boldsymbol{g}^{*}$, etc. It will be denoted for brevity by ad. Then any element $\xi \in \operatorname{Sec} A$ determines a covariant differential operator $\mathcal{L}_{\text {ad } \circ \xi}$ in the vector bundle $(W \boldsymbol{g})^{k, 2 l}$ and determines a linear operator

$$
\mathcal{L}_{\text {ad } \circ \xi}: \mathcal{W} \boldsymbol{g} \rightarrow \mathcal{W} \boldsymbol{g}
$$

such that $\mathcal{L}_{\text {ad } \circ v}=\bar{\theta}_{v}$ for $v \in \operatorname{Sec} \boldsymbol{g}$.

Lemma 4.4. The linear operator (4.4) is a differentiation of the Weil algebra $\mathcal{W} \boldsymbol{g}$.

Proof. Trivial calculations on simple tensors.

The relationships between the operators $\bar{i}_{v}, \bar{d}, \bar{\theta}_{v}, \mathcal{L}_{\text {ad } \circ \xi}$ are the following:

$$
\begin{gathered}
\mathcal{L}_{\mathrm{ad} \circ \xi} \circ \bar{d}=\bar{d} \circ \mathcal{L}_{\mathrm{ad} \circ \xi}, \\
\bar{i}_{v} \circ \bar{d}+\bar{d} \circ \bar{i}_{v}=\bar{\theta}_{v} .
\end{gathered}
$$


Indeed, $\varepsilon_{1}:=\mathcal{L}_{\mathrm{ad} \circ \xi} \circ \bar{d}-\bar{d} \circ \mathcal{L}_{\mathrm{ad} \circ \xi}$ is an antiderivation, whereas $\varepsilon_{2}:=\bar{i}_{v} \circ \bar{d}+\bar{d} \circ \bar{i}_{v}$ is a derivation, of the Weil algebra $\mathcal{W} \boldsymbol{g}$, therefore to prove (4.5) and (4.6) it is sufficient to show that $\varepsilon_{1}=0$ and $\varepsilon_{2}=\bar{\theta}_{v}$ on the cross-sections $\Psi \otimes 1$ and $1 \otimes \Psi, \Psi \in \operatorname{Sec} \boldsymbol{g}^{*}$, which is trivial.

Proposition 4.5. For $\Theta \in \operatorname{Sec} \bigwedge^{k} \boldsymbol{g}^{*} \otimes \bigvee^{l} \boldsymbol{g}^{*}$, the following equality holds:

$$
d^{A} \overline{\boldsymbol{k}}(\Theta)=\overline{\boldsymbol{k} d}(\Theta)+\frac{1}{k ! \cdot l !} \cdot\left\langle d^{g} \Theta,(\omega \wedge \cdots \wedge \omega) \otimes\left(d^{g} \omega \vee \cdots \vee d^{g} \omega\right)\right\rangle
$$

where $d^{g} \Theta \in \Omega_{A}^{1}\left(M ; \bigwedge^{k} \boldsymbol{g}^{*} \otimes \bigvee^{l} \boldsymbol{g}^{*}\right)$ ( $\Theta$ is treated as a 0 -form).

Proof. We first prove the equalities

$$
\begin{gathered}
\overline{\boldsymbol{k} d}(\Psi \otimes 1)=\frac{1}{k !} \cdot\left\langle\Psi, d^{g}(\omega \wedge \cdots \wedge \omega)\right\rangle \\
\left\langle d^{g}(\Psi \otimes \Gamma), \omega \wedge \cdots \wedge \omega \otimes d^{g} \omega \vee \cdots \vee d^{g} \omega\right\rangle \\
=\left\langle d^{g} \Psi, \omega \wedge \cdots \wedge \omega\right\rangle \wedge\left\langle\Gamma, d^{g} \omega \vee \cdots \vee d^{g} \omega\right\rangle+ \\
+(-1)^{k}\langle\Psi, \omega \wedge \cdots \wedge \omega\rangle \wedge\left\langle d^{g} \Gamma, d^{g} \omega \vee \cdots \vee d^{g} \omega\right\rangle
\end{gathered}
$$

for $\Psi \in \operatorname{Sec} \bigwedge^{k} \boldsymbol{g}^{*}$ and $\Gamma \in \operatorname{Sec} \bigvee^{l} \boldsymbol{g}^{*}$. Thanks to the linearity of both sides with respect to $\Psi$, it is sufficient to show (4.7) on a simple tensor $\Psi$ of the form $\Psi=\psi_{1} \wedge \cdots \wedge \psi_{k}$ where $\psi_{i} \in \operatorname{Sec} \boldsymbol{g}^{*}$. Let $x \in M$ and $v_{i} \in A_{\mid x}$; by $[\mathrm{K} 7,(2.2)]$ we get

$$
\begin{aligned}
& \frac{1}{k !} \cdot\left\langle\psi_{1} \wedge \cdots \wedge \psi_{k}, d^{g}(\omega \wedge \cdots \wedge \omega)\right\rangle\left(x ; v_{1} \wedge \cdots \wedge v_{k+1}\right) \\
& =\frac{1}{(k-1) !} \cdot\langle\psi_{1} \wedge \cdots \wedge \psi_{k}, d^{g} \omega \wedge \underbrace{\omega \wedge \cdots \wedge \omega}_{k-1 \text { times }}\rangle\left(x ; v_{1} \wedge \cdots \wedge v_{k+1}\right) \\
& =\frac{1}{2 \cdot(k-1) !} \cdot \sum_{\sigma} \operatorname{sgn} \sigma \cdot\left\langle\psi_{1 x} \wedge \cdots \wedge \psi_{k x}, d^{g} \omega\left(x ; v_{\sigma(1)} \wedge v_{\sigma(2)}\right)\right. \\
& \left.\wedge \omega\left(x ; v_{\sigma(3)}\right) \wedge \cdots \wedge \omega\left(x ; v_{\sigma(k+1)}\right)\right\rangle \\
& =\frac{1}{2 \cdot(k-1) !} \cdot \sum_{\sigma} \operatorname{sgn} \sigma \cdot \sum_{i}(-1)^{i+1}(d \omega)^{\vee}\left(\psi_{i}\right)\left(x ; v_{\sigma(1)} \wedge v_{\sigma(2)}\right) \text {. } \\
& \cdot\left\langle\psi_{1} \wedge \cdots \widehat{i} \cdots \wedge \psi_{k}, \omega \wedge \cdots \wedge \omega\right\rangle\left(x ; v_{\sigma(3)} \wedge \cdots \wedge v_{\sigma(k+1)}\right) \\
& =\sum_{i}(-1)^{i+1}(d \omega)^{\vee}\left(\psi_{i}\right) \wedge \omega^{\wedge}\left(\psi_{1} \wedge \cdots \widehat{i} \cdots \wedge \psi_{k}\right)\left(x ; v_{1} \wedge \cdots \wedge v_{k+1}\right) \\
& =\left(\sum_{i}(-1)^{i+1} \omega^{\wedge}\left(\psi_{1} \wedge \cdots \widehat{i} \cdots \wedge \psi_{k}\right) \wedge(d \omega)^{\vee}\left(\psi_{i}\right)\right)\left(x ; v_{1} \wedge \cdots \wedge v_{k+1}\right) \\
& =(\overline{\boldsymbol{k} d}(\Psi \otimes 1))\left(x ; v_{1} \wedge \cdots \wedge v_{k+1}\right) .
\end{aligned}
$$

Equality (4.8) easily follows from the definitions. To prove the Proposition it is sufficient to consider $\Theta=\Psi \otimes \Gamma$ for $\Psi \in \operatorname{Sec} \bigwedge^{k} \boldsymbol{g}^{*}$ and $\Gamma \in \operatorname{Sec} \bigvee^{l} \boldsymbol{g}^{*}$ and use Corollary 2.2 and the above equalities.

Let $(\mathcal{W} \boldsymbol{g})_{I^{0}}^{k, 2 l}$ denote the space of cross-sections invariant with respect to the adjoint representation of $A$ on $(W \boldsymbol{g})^{k, 2 l}=\bigwedge^{k} \boldsymbol{g}^{*} \otimes \bigvee^{l} \boldsymbol{g}^{*}$. Put $(\mathcal{W} \boldsymbol{g})_{I^{0}}=\bigoplus_{k, l \geq 0}(\mathcal{W} \boldsymbol{g})_{I^{0}}^{k, 2 l} \cdot(\mathcal{W} \boldsymbol{g})_{I^{0}}^{0,0}$ is equal to $\Omega_{b}^{0}(M, \mathcal{F})$ the space of $\mathcal{F}$-basic functions (i.e. constant along leaves of $\mathcal{F}$ ).

The following easily follows from Lemma 4.4. 
COROLlary 4.6. $(\mathcal{W g})_{I^{0}}$ is a subalgebra of the Weil algebra $\mathcal{W g}$.

Equality (4.5) implies that $\bar{d}$ maps invariant elements of $\mathcal{W g}$ into invariant ones, defining an antiderivation of algebras $\bar{d}_{0}:(\mathcal{W} \boldsymbol{g})_{I^{0}} \rightarrow(\mathcal{W} \boldsymbol{g})_{I^{0}}$, whereas Proposition 4.5 yields that $\overline{\boldsymbol{k}}$ restricted to the invariant cross-sections $\overline{\boldsymbol{k}}_{0}:(\mathcal{W} \boldsymbol{g})_{I^{0}} \rightarrow \Omega_{A}(M)$ commutes with the differentials $\bar{d}_{0}$ and $d^{A}$, giving a homomorphism $\overline{\boldsymbol{k}}_{0 \#}: H\left((\mathcal{W} \boldsymbol{g})_{I^{0}}, \bar{d}_{0}\right) \rightarrow H_{A}(M)$ of algebras. However, $\overline{\boldsymbol{k}}_{0 \#}$ is unimportant because the space $\left((\mathcal{W} \boldsymbol{g})_{I^{0}}, \bar{d}_{0}\right)$ is trivial:

$$
H\left((\mathcal{W} \boldsymbol{g})_{I^{0}}, \bar{d}_{0}\right) \cong(\mathcal{W} \boldsymbol{g})_{I^{0}}^{0} \quad\left(=(\mathcal{W} \boldsymbol{g})_{I^{0}}^{0,0}=\Omega_{b}^{0}(M, \mathcal{F})\right) .
$$

Indeed, a chain homotopy joining id to 0 is defined by the family of invariant linear homomorphisms of vector bundles $c^{k, l}:(W \boldsymbol{g})^{k, 2 l} \rightarrow(W \boldsymbol{g})^{k+1,2(l-1)}$ such that $c^{k, 0}=0$ and, for $l>0, c^{k, l}\left(w_{1}^{*} \wedge \cdots \wedge w_{k}^{*} \otimes \Gamma_{1} \vee \cdots \vee \Gamma_{l}\right)=\frac{(-1)^{k}}{k+l} \cdot \sum_{s=1}^{l} w_{1}^{*} \wedge \cdots \wedge w_{k}^{*} \wedge \Gamma_{s} \otimes \Gamma_{1} \vee$ $\cdots \widehat{s} \cdots \vee \Gamma_{l}, w_{i}^{*}, \Gamma_{j} \in \boldsymbol{g}_{\mid x}^{*}, x \in M$.

\section{The change of variables in $W g_{\mid x}$}

Proposition 5.1. There exists exactly one isomorphism $\varphi_{x}: W \boldsymbol{g}_{\mid x} \rightarrow W \boldsymbol{g}_{\mid x}$ of algebras of degree 0 such that

1. $\varphi_{x}(1)=1$,

2. $\varphi_{x}\left(w^{*} \otimes 1\right)=w^{*} \otimes 1$,

3. $\varphi_{x}\left(1 \otimes w^{*}\right)=1 \otimes w^{*}-\delta_{x} w^{*} \otimes 1, \quad w^{*} \in \boldsymbol{g}_{\mid x}^{*}$, where $\delta_{x}$ denotes the differential in the algebra $\wedge \boldsymbol{g}_{\mid x}^{*}$, defined in [K7, (2.5)].

Proof. We recall that $\delta_{x}$ is equal to minus the classical Chevalley-Eilenberg differential. The uniqueness is evident. To prove the existence, take two uniquely determined linear mappings $\widetilde{\varphi}_{x^{+}}, \widetilde{\varphi}_{x^{-}}: \bigvee \boldsymbol{g}_{\mid x}^{*} \rightarrow W \boldsymbol{g}_{\mid x}$, satisfying the conditions

(i) $\widetilde{\varphi}_{x^{ \pm}}(1)=1$,

(ii) $\widetilde{\varphi}_{x^{ \pm}}\left(\Gamma_{1 x} \vee \cdots \vee \Gamma_{l x}\right)=\prod_{i=1}^{l}\left(1 \otimes \Gamma_{i x} \pm \delta_{x}\left(\Gamma_{i x}\right) \otimes 1\right), \quad \Gamma_{i x} \in \boldsymbol{g}_{\mid x}^{*}, \quad l \geq 1$.

They are homomorphisms of algebras of degree 0 [the degree $\Gamma_{x}=2$ for $\Gamma_{x} \in \boldsymbol{g}_{\mid x}^{*}$ ] and

$$
\widetilde{\varphi}_{x^{ \pm}}\left[\bigvee^{l} \boldsymbol{g}_{\mid x}^{*}\right] \subset \bigoplus_{m=0}^{l}\left(\bigwedge^{2(l-m)} \boldsymbol{g}_{\mid x}^{*} \otimes \bigvee^{m} \boldsymbol{g}_{\mid x}^{*}\right)
$$

Clearly, there exist two linear mappings $\varphi_{x^{+}}, \varphi_{x^{-}}: W \boldsymbol{g}_{\mid x} \rightarrow W \boldsymbol{g}_{\mid x}$ such that $\left(\mathrm{iii}_{ \pm}\right) \varphi_{x^{ \pm}}\left(\Psi_{x} \otimes \Gamma_{x}\right)=\Psi_{x} \otimes 1 \cdot \widetilde{\varphi}_{x^{ \pm}}\left(\Gamma_{x}\right), \quad \Psi_{x} \in \bigwedge \boldsymbol{g}_{\mid x}^{*}, \quad \Gamma_{x} \in \bigvee \boldsymbol{g}_{\mid x}^{*}$.

They are homomorphisms of algebras of degree 0 (which can be easily proved by considering tensors bihomogeneous only), and fulfil the property

$\left(\mathrm{iv}_{ \pm}\right) \varphi_{x^{ \pm}}\left(1 \otimes w^{*}\right)=1 \otimes w^{*} \pm \delta_{x} w^{*} \otimes 1, \quad w^{*} \in \boldsymbol{g}_{\mid x}^{*}$.

To end the proof, put $\varphi_{x}:=\varphi_{x^{-}}$. To see that $\varphi_{x}$ is an isomorphism, we check the equalities $\varphi_{x^{-}} \circ \varphi_{x^{+}}=\mathrm{id}, \varphi_{x^{+}} \circ \varphi_{x^{-}}=\mathrm{id}$. Both sides of these are homomorphisms of algebras, therefore it is sufficient to check them on the generators, which is trivial.

All the isomorphisms $\varphi_{x}, x \in M$, establish an isomorphism of algebras

$$
\varphi: \mathcal{W g} \rightarrow \mathcal{W g}
$$


$\varphi(\Theta)(x)=\varphi_{x}\left(\Theta_{x}\right), \quad x \in M$. By the proof above,

$$
\varphi^{-1}(\Psi \otimes 1)=\Psi \otimes 1 \text { and } \varphi^{-1}(1 \otimes \Psi)=1 \otimes \Psi+\delta \Psi \otimes 1
$$

for $\Psi \in \operatorname{Sec} \boldsymbol{g}^{*}$. Moreover, $\varphi_{x}$ establish linear homomorphisms of vector bundles

$$
\varphi^{l}: \bigwedge \boldsymbol{g}^{*} \otimes \bigvee^{\leq l} \boldsymbol{g}^{*} \cong \boldsymbol{g}^{*} \otimes \bigvee^{\leq l} \boldsymbol{g}^{*}, \quad \varphi^{k, 2 l}:(W \boldsymbol{g})^{k, 2 l} \rightarrow \bigoplus_{m=0}^{l}(W \boldsymbol{g})^{k+2(l-m), 2 m}
$$

The following equality holds:

$$
\varphi^{k, 2 l} \circ(\Psi \otimes \Gamma)=\Psi \otimes 1 \cdot\left(\varphi^{0,2 l} \circ(1 \otimes \Gamma)\right), \quad \Psi \in \operatorname{Sec} \bigwedge^{k} \boldsymbol{g}^{*}, \quad \Gamma \in \operatorname{Sec} \bigvee^{l} \boldsymbol{g}^{*}
$$

Proposition 5.2. $\varphi^{k, 2 l}$ is an invariant homomorphism.

Proof. We have to prove only that

$$
\mathcal{L}_{\text {ad } \circ \xi}\left(\varphi^{k, 2 l} \circ \Theta\right)=\varphi^{k, 2 l} \circ \mathcal{L}_{\text {ad } \circ \xi} \Theta
$$

for $\xi \in \operatorname{Sec} A$ and $\Theta \in \operatorname{Sec} \bigwedge^{k} \boldsymbol{g}^{*} \otimes \bigvee^{l} \boldsymbol{g}^{*}$. First, we check the equality

$$
\mathcal{L}_{\mathrm{ad} \circ \xi}(\delta \Gamma)=\delta\left(\mathcal{L}_{\mathrm{ad} \circ \xi} \Gamma\right)
$$

for $\Gamma \in \operatorname{Sec} \boldsymbol{g}^{*}$. To this end, take $\nu_{1}, \nu_{2} \in \operatorname{Sec} \boldsymbol{g}$. The Jacobi identity implies

$$
\begin{aligned}
& \left\langle\mathcal{L}_{\mathrm{ad} \circ \xi}(\delta \Gamma), \nu_{1} \wedge \nu_{2}\right\rangle=(\gamma \circ \xi)\left\langle\Gamma, \llbracket \nu_{1}, \nu_{2} \rrbracket\right\rangle-\left\langle\Gamma, \llbracket \llbracket \xi, \nu_{1} \rrbracket, \nu_{2} \rrbracket\right\rangle-\left\langle\Gamma, \llbracket \nu_{1}, \llbracket \xi, \nu_{2} \rrbracket \rrbracket\right\rangle \\
& =\left\langle\mathcal{L}_{\text {ad } \circ \xi} \Gamma, \llbracket \nu_{1}, \nu_{2} \rrbracket\right\rangle=\left\langle\delta\left(\mathcal{L}_{\text {ad } \circ \xi} \Gamma\right), \nu_{1} \wedge \nu_{2}\right\rangle .
\end{aligned}
$$

Next, we obtain

$$
\mathcal{L}_{\mathrm{ad} \circ \xi}\left(\varphi^{0,2} \circ(1 \otimes \Gamma)\right)=1 \otimes \mathcal{L}_{\mathrm{ad} \circ \xi} \Gamma-\delta\left(\mathcal{L}_{\mathrm{ad} \circ \xi} \Gamma\right) \otimes 1=\varphi^{0,2} \circ\left(1 \otimes \mathcal{L}_{\mathrm{ad} \circ \xi} \Gamma\right),
$$

for $\Gamma \in \operatorname{Sec} \boldsymbol{g}^{*}$. As usual, to obtain (5.2) it is sufficient to consider $\Theta=\psi_{1} \wedge \cdots \wedge \psi_{k} \otimes$ $\Gamma_{1} \vee \cdots \vee \Gamma_{l}, \psi_{i}, \Gamma_{j} \in \operatorname{Sec} \boldsymbol{g}^{*}$. By (5.1), (5.4) and Lemma 4.4 we obtain

$$
\begin{aligned}
& \mathcal{L}_{\mathrm{ad} \circ \xi}\left(\varphi^{k, 2 l} \circ\left(\psi_{1} \wedge \cdots \wedge \psi_{k} \otimes \Gamma_{1} \vee \cdots \vee \Gamma_{l}\right)\right) \\
& =\sum_{i} \psi_{1} \wedge \cdots \wedge \mathcal{L}_{\mathrm{ad} \circ \xi}\left(\psi_{i}\right) \wedge \cdots \wedge \psi_{k} \otimes 1 \cdot \varphi^{0,2 l} \circ\left(1 \otimes \Gamma_{1} \vee \cdots \vee \Gamma_{l}\right) \\
& \left.+\psi_{1} \wedge \cdots \wedge \psi_{k} \otimes 1 \cdot \sum_{i} \varphi^{0,2} \circ\left(1 \otimes \Gamma_{1}\right) \cdot \ldots \cdot \varphi^{0,2} \circ\left(1 \otimes \mathcal{L}_{\mathrm{ad} \circ \xi} \Gamma_{i}\right)\right) \cdot \ldots \\
& \cdots \cdot\left(\varphi^{0,2} \circ\left(1 \otimes \Gamma_{l}\right)\right. \\
& =\varphi^{k, 2 l} \circ\left(\sum_{i} \psi_{1} \wedge \cdots \wedge \mathcal{L}_{\mathrm{ad} \circ \xi}\left(\psi_{i}\right) \wedge \cdots \wedge \psi_{k} \otimes \Gamma_{1} \vee \cdots \vee \Gamma_{l}\right. \\
& \left.\quad+\psi_{1} \wedge \cdots \wedge \psi_{k} \otimes \sum_{i} \Gamma_{1} \vee \cdots \vee \mathcal{L}_{\mathrm{ad} \circ \xi}\left(\Gamma_{i}\right) \vee \cdots \vee \Gamma_{l}\right) \\
& =\varphi^{k, 2 l} \circ \mathcal{L}_{\mathrm{ad} \circ \xi}\left(\psi_{1} \wedge \cdots \wedge \psi_{k} \otimes \Gamma_{1} \vee \cdots \vee \Gamma_{l}\right) .
\end{aligned}
$$

Corollary 5.3. (1) $\varphi^{l}: \bigwedge \boldsymbol{g}^{*} \otimes \bigvee^{\leq l} \boldsymbol{g}^{*} \rightarrow \bigwedge \boldsymbol{g}^{*} \otimes \bigvee^{\leq l} \boldsymbol{g}^{*}$ is an invariant isomorphism of vector bundles, therefore $\left(\varphi^{l}\right)^{-1}$ is invariant, too.

(2) $\varphi(\Theta)$ is an invariant element of $\mathcal{W} \boldsymbol{g}$ whenever $\Theta \in \mathcal{W} \boldsymbol{g}$ is invariant.

(3) $\varphi_{0}:(\mathcal{W g})_{I^{0}} \rightarrow(\mathcal{W g})_{I^{0}}$, the restriction of $\varphi$ to invariant cross-sections, is an isomorphism of algebras. 
6. Operators $i_{\nu}, d, \theta_{\nu}$ and their properties. We define the fundamental operators $i_{\nu}, d, \theta_{\nu}$ in $\mathcal{W} \boldsymbol{g}$ in such a way that the following diagram commutes:

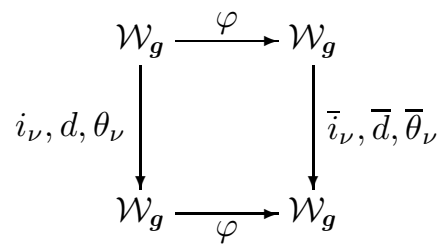

Of course, one can follow this procedure on each level of $x \in M$ to obtain the operators $i_{x, v}, d_{x}, \theta_{x, v}$ on $W \boldsymbol{g}_{\mid x}$, with the relations $i_{x, \nu_{x}}\left(\theta_{x}\right)=i_{\nu}(\Theta)(x)$, etc.

Proposition 6.1. The fundamental properties of the operators $i_{\nu}, d, \theta_{\nu}$ are as follows:

(1) $\theta_{\nu}=\bar{\theta}_{\nu}$, (2) $\mathcal{L}_{\mathrm{ad} \circ \xi} \circ d=d \circ \mathcal{L}_{\mathrm{ad} \circ \xi}$, (3) $i_{\nu} \circ d+d \circ i_{\nu}=\theta_{\nu}$.

Proof. To prove (1) we recall that $\theta_{\nu}$ and $\bar{\theta}_{\nu}$ are derivations, therefore it is sufficient to show the equality $\theta_{\nu}(\Theta)=\bar{\theta}_{\nu}(\Theta)$ for the cross-sections $\Theta=\Psi \otimes 1$ and $\Theta=1 \otimes \Psi, \Psi \in$ $\operatorname{Sec} \boldsymbol{g}^{*}$ which is easy to obtain. Equality (2) is evident because $\mathcal{L}_{\text {ad } \circ \xi}$ commutes with $\varphi, \varphi^{-1}$ and $\bar{d}$. Analogously we check (3).

Proposition 6.2. (1) $i_{\nu}$ is an antiderivation of degree -1 defined uniquely by the conditions $i_{\nu}(\Psi \otimes 1)=i_{\nu} \Psi$, and $i_{\nu}(1 \otimes \Psi)=0, \Psi \in \operatorname{Sec} \boldsymbol{g}^{*}$. It has the property

(i) $i_{\nu}(\Psi \otimes \Gamma)=i_{\nu}(\Psi) \otimes \Gamma$ for $\Psi \in \operatorname{Sec} \bigwedge^{k} \boldsymbol{g}^{*}, \quad \Gamma \in \operatorname{Sec} \bigvee^{l} \boldsymbol{g}^{*}$.

(2) $d$ is an antiderivation of degree +1 defined uniquely by the conditions

$\left(1^{\circ}\right) d(\Psi \otimes 1)=1 \otimes \Psi+\delta \Psi \otimes 1$,

$\left(\mathscr{2}^{\circ}\right) d(1 \otimes \Psi)$ is an element of $(\mathcal{W} \boldsymbol{g})^{1,2}=\operatorname{Sec}\left(\boldsymbol{g}^{*} \otimes \boldsymbol{g}^{*}\right)$ such that $i_{\nu} \circ d(1 \otimes \Psi)=\theta_{\nu} \Psi$ for $\nu \in \operatorname{Sec} \boldsymbol{g}$.

Proof. Trivially follows from Prop. 6.1.

The families of operators $i_{x, \nu_{x}}, d_{x}, \theta_{x, \nu_{x}}$, indexed by $x \in M$, give rise, for $k, l \geq 0$, to the linear homomorphisms of vector bundles

$$
\begin{aligned}
i_{\nu}^{k, 2 l}: & (W \boldsymbol{g})^{k, 2 l} \rightarrow(W \boldsymbol{g})^{k-1,2 l}, \quad \theta_{\nu}^{k, 2 l}:(W \boldsymbol{g})^{k, 2 l} \rightarrow(W \boldsymbol{g})^{k, 2 l}, \\
& d_{\nu}^{k, 2 l}:(W \boldsymbol{g})^{k, 2 l} \rightarrow(W \boldsymbol{g})^{k+1,2 l} \oplus(W \boldsymbol{g})^{k-1,2(l+1)} .
\end{aligned}
$$

Proposition 6.1(2) implies

COROLlary 6.3. $d$ maps invariant elements of $\mathcal{W} \boldsymbol{g}$ into invariant ones, defining an antiderivation $d_{0}:(\mathcal{W} \boldsymbol{g})_{I^{0}} \rightarrow(\mathcal{W} \boldsymbol{g})_{I^{0}}$.

$\varphi_{0}:(\mathcal{W} \boldsymbol{g})_{I^{0}} \rightarrow(\mathcal{W} \boldsymbol{g})_{I^{0}}$ commuting with $\bar{d}_{0}$ and $d_{0}$ gives an isomorphism

$$
\varphi_{0 \#}: H\left((\mathcal{W} \boldsymbol{g})_{I^{0}}, d_{0}\right) \stackrel{\cong}{\rightarrow} H\left((\mathcal{W} \boldsymbol{g})_{I^{0}}, \bar{d}_{0}\right),
$$

therefore $H\left((\mathcal{W} \boldsymbol{g})_{I^{0}}, d_{0}\right)$ is trivial according to (4.9).

The cross-sections $\Theta \in \mathcal{W} \boldsymbol{g}$, for which $i_{\nu} \Theta=0$ for each $\nu \in \operatorname{Sec} \boldsymbol{g}$, are called horizontal (or more precisely, $\boldsymbol{g}$-horizontal). Since $i_{\nu}$ is an antiderivation, all horizontal cross-sections form a subalgebra of $\mathcal{W} \boldsymbol{g}$ denoted by $(\mathcal{W} \boldsymbol{g})_{i}$. This construction can be performed on each level of $x \in M$ to obtain the algebra $\left(\mathcal{W} \boldsymbol{g}_{\mid x}\right)_{i}$. Of course, $\Theta \in(\mathcal{W} \boldsymbol{g})_{i} \Longleftrightarrow \Theta_{x} \in\left(\mathcal{W} \boldsymbol{g}_{\mid x}\right)_{i}$ for each $x \in M$. 
Lemma 6.4. $(\mathcal{W} \boldsymbol{g})_{i}=\bigoplus^{l \geq 0}(\mathcal{W} \boldsymbol{g})^{0,2 l} \cong \bigoplus^{l \geq 0} \mathrm{Sec}^{l} \boldsymbol{g}^{*}$; equivalently,

$$
\left(\mathcal{W} \boldsymbol{g}_{\mid x}\right)_{i}=\mathbb{R} \otimes \bigvee \boldsymbol{g}_{\mid x}^{*}
$$

for each $x \in M$. In consequence, each nontrivial homogeneous element of $(\mathcal{W} \boldsymbol{g})_{i}$ has an even degree.

Proof. Let $\psi_{x}=\sum_{j} \psi_{x}^{j} \otimes \Gamma_{x}^{j} \in\left(\mathcal{W} \boldsymbol{g}_{\mid x}\right)_{i}, \psi_{x}^{j} \in \bigwedge \boldsymbol{g}_{\mid x}^{*}$ and $\Gamma_{x}^{j} \in \bigvee \boldsymbol{g}_{\mid x}^{*}$; we can assume the linear independence of $\Gamma_{x}^{j}[\mathrm{G}, \mathrm{p} .7]$. Since $0=i_{x, v}\left(\psi_{x}\right)=\sum_{j} i_{x, v}\left(\psi_{x}^{j}\right) \otimes \Gamma_{x}^{j}$, therefore $i_{x, v}\left(\psi_{x}^{j}\right)=0$ for each $v \in \boldsymbol{g}_{\mid x}$. But $\bigcap_{v \in \boldsymbol{g}_{\mid x}} \operatorname{ker} i_{x, v}=\mathbb{R}[\mathrm{G}$, p. 117], then we obtain $\psi_{x}^{j}=r^{j} \in \mathbb{R} \subset \bigwedge \boldsymbol{g}_{\mid x}^{*}$.

\section{Lemma 4.4 and Proposition 6.1 yield}

Corollary 6.5. $d$ maps invariant and (simultaneously) horizontal elements of $\mathcal{W g}$ into such elements, defining the antiderivation $d_{i, 0}:\left(\mathcal{W} \boldsymbol{g}_{\mid x}\right)_{i, I^{0}} \rightarrow\left(\mathcal{W} \boldsymbol{g}_{\mid x}\right)_{i, I^{0}}$.

7. The mapping $\boldsymbol{k}$. Put $\boldsymbol{k}=\overline{\boldsymbol{k}} \circ \varphi: \mathcal{W} \boldsymbol{g} \rightarrow \Omega_{A}(M)$, which is a homomorphism of algebras.

Lemma 7.1. $\boldsymbol{k}_{0}:(\mathcal{W} \boldsymbol{g})_{I^{0}} \rightarrow \Omega_{A}(M)$, the restriction of $\boldsymbol{k}$ to the invariant crosssections commutes with the differentials $d_{0}$ and $d^{A}$.

Proof. For $\Theta \in(\mathcal{W} \boldsymbol{g})_{I^{0}}$ we have, by Corollary 5.3(2), $d^{A} \circ \boldsymbol{k}_{0}(\Theta)=d^{A} \circ \overline{\boldsymbol{k}}_{0} \circ \varphi(\Theta)=$ $\overline{\boldsymbol{k}} \circ \bar{d} \circ \varphi(\Theta)=\boldsymbol{k}_{0} \circ d_{0}(\Theta)$.

Proposition 7.2. $\boldsymbol{k}(\Psi \otimes \Gamma)=\omega^{\wedge}(\Psi) \wedge \Omega^{\vee}(\Gamma)$.

Proof. $\boldsymbol{k}(\Psi \otimes \Gamma)=\boldsymbol{k}(\Psi \otimes 1 \cdot 1 \otimes \Gamma)=\boldsymbol{k}(\Psi \otimes 1) \wedge \boldsymbol{k}(1 \otimes \Gamma)=\omega^{\wedge}(\Psi) \wedge \boldsymbol{k}(1 \otimes \Gamma)$. It remains to verify that $\boldsymbol{k}(1 \otimes \Gamma)=\Omega^{\vee}(\Gamma)$. But the mappings $\Gamma \mapsto \boldsymbol{k}(1 \otimes \Gamma)$ and $\Gamma \mapsto \Omega^{\vee}(\Gamma)$ are homomorphisms of algebras such that $1 \mapsto 1$, therefore it is sufficient to check the equality for $\Gamma=\Psi \in \operatorname{Sec} \boldsymbol{g}^{*}$. Formula $[\mathrm{K} 7,(2.6)]$ yields

$$
\begin{aligned}
\boldsymbol{k}(1 \otimes \Psi) & =\overline{\boldsymbol{k}} \circ \varphi(1 \otimes \Psi)=\overline{\boldsymbol{k}}(1 \otimes \Psi-\delta \Psi \otimes 1) \\
& =(d \omega)^{\vee}(\Psi)-\omega^{\wedge}(\delta \Psi)=\left\langle\Psi, d^{g} \omega\right\rangle-\omega^{\wedge}(\delta \Psi)=\Omega^{\vee}(\Psi) .
\end{aligned}
$$

Proposition 7.3. $i_{\nu} \circ \boldsymbol{k}=\boldsymbol{k} \circ i_{\nu}$ for $\nu \in \operatorname{Sec} \boldsymbol{g}$.

Proof. By the horizontality of the forms in $\operatorname{Im} \Omega^{\vee}$ [which easily follows from the horizontality of $\Omega]$, the standard property of the substitution operator $i_{\nu}: \Omega_{A}(M) \rightarrow$ $\Omega_{A}(M)$, equality [K7, (2.3)] and Prop. 6.2(1)(i) above, we get, for $\Psi \in \operatorname{Sec} \wedge^{k} \boldsymbol{g}^{*}$ and $\Gamma \in \bigoplus_{l \geq 0} \operatorname{Sec} \bigvee^{l} \boldsymbol{g}^{*}$

$$
\begin{aligned}
& i_{\nu} \circ \boldsymbol{k}(\Psi \otimes \Gamma)=i_{\nu}\left(\omega^{\wedge}(\Psi) \wedge \Omega^{\vee}(\Gamma)\right)+(-1)^{k} \omega^{\wedge}(\Psi) \wedge i_{\nu}\left(\Omega^{\vee}(\Gamma)\right) \\
& =\omega^{\wedge}\left(i_{\nu}(\Psi)\right) \wedge \Omega^{\vee}(\Gamma)=\boldsymbol{k}\left(i_{\nu}(\Psi) \otimes \Gamma\right)=\boldsymbol{k}\left(i_{\nu}(\Psi \otimes \Gamma)\right) .
\end{aligned}
$$

Our proposition now follows from the linearity of $\boldsymbol{k}$ and $i_{\nu}$.

8. The Chern-Weil homomorphism of regular Lie algebroids, revisited. As a simple consequence of Proposition 7.2 we obtain the Chern-Weil homomorphism of regular Lie algebroids constructed earlier in [K2]. 
$\boldsymbol{k}$ maps horizontal elements of $\mathcal{W} \boldsymbol{g}$ into horizontal real forms on $A$, giving a homomorphism of algebras $\boldsymbol{k}_{i}:(\mathcal{W} \boldsymbol{g})_{i} \rightarrow \Omega_{A, i}(M)$. This mapping is defined by the formula $\boldsymbol{k}_{i}(1 \otimes \Gamma)=\Omega^{\vee}(\Gamma), \Gamma \in \bigoplus_{l>0} \operatorname{Sec} \bigvee^{l} \boldsymbol{g}^{*}$. Consider the further restriction of $\boldsymbol{k}$, $\boldsymbol{k}_{0, i}:(\mathcal{W} \boldsymbol{g})_{I^{0}, i} \rightarrow \Omega_{A, i}(M)$ where $(\mathcal{W} \boldsymbol{g})_{I^{0}, i}$ denotes the algebra of elements horizontal and invariant simultaneously. We prove that

$$
d \mid(\mathcal{W} \boldsymbol{g})_{I^{0}, i}=0
$$

Let $0 \neq \Theta \in(\mathcal{W} \boldsymbol{g})_{I^{0}, i}$. By Corollary 6.5, $d \Theta \in(\mathcal{W} \boldsymbol{g})_{I^{0}, i}$. But $\Theta$ has an even degree (see Lemma 6.4), whereas $d$ is an antiderivation of degree +1 , therefore $d \Theta$ has an odd degree. Using Lemma 6.4 once again, we assert that $d \Theta=0$.

According to Lemma 7.1 and (8.1), the forms in $\operatorname{Im} \boldsymbol{k}_{0, i}$ are $d^{A}$-closed. The isomorphism $\lambda_{*}: \Omega_{A, i}(M) \rightarrow \Omega_{E}(M)\left(\left[\mathrm{K} 7\right.\right.$, sec. 2]) maps $d^{A}$-closed forms into $d^{E}$-closed forms, see $[K 7,(2.1)]$. By the above, there exists a homomorphism of algebras

$$
\bigoplus_{l \geq 0}\left(\operatorname{Sec}^{l} \boldsymbol{g}^{*}\right)_{I^{0}} \rightarrow H_{E}(M), \quad \Gamma \mapsto\left[\lambda_{*}\left(\Omega^{\vee}(\Gamma)\right)\right]
$$

However, $\lambda_{*}\left(\Omega^{\vee}(\Gamma)\right)=\frac{1}{l !} \cdot\left\langle\Gamma, \lambda_{*} \Omega \vee \cdots \vee \lambda_{*} \Omega\right\rangle=\frac{1}{l !} \cdot\left\langle\Gamma, \Omega_{b} \vee \cdots \vee \Omega_{b}\right\rangle$, therefore $\left[\lambda_{*}\left(\Omega^{\vee}(\Gamma)\right)\right]=\left[\frac{1}{l !} \cdot\left\langle\Gamma, \Omega_{b} \vee \cdots \vee \Omega_{b}\right\rangle\right]=h_{A}(\Gamma)$ according to $[$ K2, Ch. 4], which means that (8.2) is the Chern-Weil homomorphism of the regular Lie algebroid $A$.

\section{PART II. SECONDARY CHARACTERISTIC HOMOMORPHISM OF PARTIALLY REGULAR LIE ALGEBROIDS}

9. Regular Lie algebroids and ideals. Take two vector bundles $F^{\prime}$ and $F$ on a paracompact manifold $M$, such that $F^{\prime} \subset F$, and define (see [K6, sec. 2]), for $k \geq 1$,

$$
I_{\bigwedge^{k} F^{\prime}}:=\bigcup_{x \in M} I_{\bigwedge^{k}{ }_{\left(F_{\mid x}^{\prime}\right)}} \subset \bigwedge F
$$

${ }^{I} \bigwedge^{k} F^{\prime}$ is a vector subbundle of $\bigwedge F$ and the space of global cross-sections $\operatorname{Sec}\left(I \bigwedge^{k} F^{\prime}\right)$ is an ideal in the algebra $\operatorname{Sec}(\bigwedge F)$; moreover, $\operatorname{Sec}\left(I_{\bigwedge^{k}{ }_{F^{\prime}}}\right)=\left(\operatorname{Sec}\left(I_{F^{\prime}}\right)^{k}, \quad k \geq 1\right.$.

Let $E^{\prime} \subset E \subset T M$ be two $C^{\infty}$ constant dimensional distributions on $M$, and suppose $E$ to be integrable. Denote by $E^{\prime \perp}$ the vector subbundle of $E^{*}$ consisting of all covectors vanishing on $E^{\prime}$. Using the above (for $F=E^{*}, F^{\prime}=E^{\prime \perp}, k=1$ ), we obtain an ideal $I$ in the algebra $\Omega_{E}(M)=\operatorname{Sec} \bigwedge E^{*}$ of tangential defferential forms, generated by 1 -forms vanishing on $E^{\prime}$. Standard calculations give the following

Theorem 9.1 (The Frobenius Theorem for subdistributions). $E^{\prime}$ is involutive if and only if the ideal $I$ is differential, i.e. $d^{E}[I] \subset I$.

Consider a regular Lie algebroid $(A, \llbracket \cdot, \cdot \rrbracket, \gamma)$ over a foliated manifold $(M, E)$ and an involutive subdistribution $E^{\prime} \subset E$. This produces a new regular Lie algebroid $\left(A^{\prime}, \llbracket \cdot, \cdot \rrbracket, \gamma \mid A^{\prime}\right)$ in which $A^{\prime}=\gamma^{-1}\left[E^{\prime}\right]$.

In the sequel, the symbols $A^{\prime \perp}$ and $E^{\prime} \perp$ are understood with respect to the canonical dualities $A^{*} \times A \rightarrow \mathbb{R}$ and $E^{*} \times E \rightarrow \mathbb{R}$ (see [K6]). Consider the ideal

$$
\operatorname{Sec}\left(I^{k}\left(A^{\prime \perp}\right) \subset \Omega_{A}(M)=\operatorname{Sec} \bigwedge A^{*}\right.
$$


which is the $k$-power of the ideal of real forms on the Lie algebroid $A$, vanishing on $A^{\prime}$. Since $\Psi \in \operatorname{Sec} A^{\perp \perp}$ if and only if $\Psi=\gamma_{*} \theta$ for some $\theta \in \operatorname{Sec} E^{\perp}$, we obtain that each form $\Psi \in \operatorname{Sec}\left(I \wedge^{k}\left(A^{\prime \perp}\right)\right)$ is globally of the form $\Psi=\sum_{i=1}^{l} \gamma_{*}\left(\theta_{i}^{1} \wedge \ldots \wedge \theta_{i}^{k}\right) \wedge \Psi_{i}$ for an integer $l, \theta_{i}^{j} \in \operatorname{Sec}\left(E^{\prime \perp}\right)$ and $\Psi_{i} \in \Omega_{A}(M)$. The above Theorem 9.1, and the equalities $d^{A} \gamma_{*}=\gamma_{*} d^{E}([\mathrm{~K} 7,(2.1)]), i_{\xi} \gamma_{*}=\gamma_{*} i_{\gamma \circ \xi}, \quad \theta_{\xi}^{A} \gamma_{*}=\gamma_{*} \theta_{\gamma \circ \xi}$ for $\xi \in \operatorname{Sec} A^{\prime}$, make the following proposition obvious:

Proposition 9.2. The ideal $\operatorname{Sec}\left(I_{\Lambda^{k}\left(A^{\prime \perp}\right)}\right)$ is closed with respect to the operators $d^{A}, i_{\xi}, \theta_{\xi}^{A}$ for $\xi \in \operatorname{Sec} A^{\prime}$.

The fact that $\bigwedge \gamma^{*}: \bigwedge E^{*} \rightarrow \bigwedge A^{*}$ is a monomorphism and the equality

$$
i_{w_{1} \wedge \cdots \wedge w_{h-k+1}}\left(\gamma_{*} \theta\right)=\gamma_{*} i_{\gamma w_{1} \wedge \cdots \wedge \gamma w_{h-k+1}} \theta
$$

for $\theta \in \Omega_{E}(M)$ and $w_{i} \in \operatorname{Sec} A$ (see also [K6] and [An]) imply

Corollary 9.3. $\theta \in \operatorname{Sec}\left(I_{\bigwedge^{k}\left(E^{\prime \perp}\right)}\right) \Longleftrightarrow \gamma_{*} \theta \in \operatorname{Sec}\left(I_{\Lambda^{k}\left(A^{\prime \perp}\right)}\right)$.

Recall that [K-T2], [K6, Rem. 4.2] by a partial connection in $A$ over $E^{\prime}$ we mean any connection $\lambda^{\prime}: E^{\prime} \rightarrow A^{\prime}$ in the regular Lie algebroid $A^{\prime}=\gamma^{-1}\left[E^{\prime}\right]$.

If $\lambda^{\prime}$ is flat, then the pair $\left(A, \lambda^{\prime}\right)$ is called a partially flat regular Lie algebroid. Any foliated principal bundle [K-T2, p. 20] gives in a natural manner a partially flat regular Lie algebroid. A connection $\lambda: E \rightarrow A$ in $A$ is said to be adapted to $\lambda^{\prime}$ when $\lambda^{\prime}=\lambda \mid E^{\prime}$ (an adapted connection always exists).

Assume that $A$ is equipped with a connection $\lambda$ and a partial connection $\lambda^{\prime}$ over $E^{\prime}$. Let $\Omega$ and $\Omega^{\prime}$ ( $\Omega_{b}$ and $\Omega_{b}^{\prime}$ ) be the curvature forms (the curvature tensors) of these connections (see [K7, Sec. 2], and [K2, 3.1.1]). From the equality $\Omega=\gamma_{*} \Omega_{b}$ and Corollary 9.3 (see also [K6]), we obtain

THEOREm 9.4. If $\lambda$ is adapted to $\lambda^{\prime}$, then

(a) $\lambda^{\prime}$ is flat if and only if $\left\langle v^{*}, \Omega_{\mid x}\right\rangle \in I_{\Lambda^{1}\left(A_{\mid x}^{\prime \perp}\right)}^{(2)}$ for any $x \in M$ and $v^{*} \in \boldsymbol{g}_{\mid x}^{*}$,

(b) $\lambda$ is basic if and only if $\left\langle v^{*}, \Omega_{\mid x}\right\rangle \in I_{\Lambda^{2}\left(A_{\mid x}^{\prime \perp}\right)}^{(2)}$ for any $x \in M$ and $v^{*} \in \boldsymbol{g}_{\mid x}^{*}$.

We now pass to the Weil algebras $W \boldsymbol{g}_{\mid x}$ and $\mathcal{W} \boldsymbol{g}$. $W \boldsymbol{g}_{\mid x}$ has a standard even decreasing filtration by ideals

$$
\mathbf{F}^{2 p}\left(W \boldsymbol{g}_{\mid x}\right):=I_{\mathbb{R} \otimes \bigvee{ }^{p} \boldsymbol{g}_{\mid x}^{*}}=\bigwedge \boldsymbol{g}_{\mid x}^{*} \otimes \bigvee^{\geq p} \boldsymbol{g}_{\mid x}^{*}
$$

These, for all $x \in M$, define an even decreasing filtration by ideals of the Weil algebra $\mathcal{W g}$

$$
\mathbf{F}^{2 p}(\mathcal{W} \boldsymbol{g}):=\left\{\Theta \in \mathcal{W} \boldsymbol{g} ; \forall x \in M, \Theta_{x} \in \mathbf{F}^{2 p}\left(W \boldsymbol{g}_{\mid x}\right)\right\}=\bigoplus_{l \geq p} \operatorname{Sec}\left(\bigwedge \boldsymbol{g}^{*} \otimes \bigvee^{l} \boldsymbol{g}^{*}\right)
$$

The algebras $\bigwedge A_{\mid x}^{*}$ and $\bigwedge E_{\mid x}^{*}$ possess decreasing filtrations by ideals

$$
\mathbf{F}^{p}\left(\bigwedge A_{\mid x}^{*}\right)=I_{\bigwedge^{p}\left(A_{\mid x}^{\prime \perp}\right)}, \quad \mathbf{F}^{p}\left(\bigwedge E_{\mid x}^{*}\right)=I \bigwedge^{p}\left(E_{\mid x}^{\prime \perp}\right)
$$

which determine decreasing filtrations by ideals of the algebras $\Omega_{A}(M)$ and $\Omega_{E}(M)$

$$
\begin{aligned}
& \mathbf{F}^{p}\left(\Omega_{A}(M)\right)=\left\{\Psi \in \Omega_{A}(M) ; \forall x \in M, \Psi_{x} \in \mathbf{F}^{p}\left(\bigwedge A_{\mid x}^{*}\right)\right\}=\operatorname{Sec} I \bigwedge^{p}\left(A^{\prime \perp}\right) \\
& \mathbf{F}^{p}\left(\Omega_{E}(M)\right)=\left\{\Theta \in \Omega_{E}(M) ; \forall x \in M, \Theta_{x} \in \mathbf{F}^{p}\left(\bigwedge E_{\mid x}^{*}\right)\right\}=\operatorname{Sec} I \bigwedge^{p}\left(E^{\prime \perp}\right)
\end{aligned}
$$


Proposition 9.5. Let $\left(A, \lambda^{\prime}\right)$ be a partially flat regular Lie algebroid and $\lambda$ an adapted connection. Then the homomorphism $\boldsymbol{k}: \mathcal{W} \boldsymbol{g} \rightarrow \Omega_{A}(M)$ (defined in Part I for $\lambda$ ) is filtration-preserving in the sense that $\boldsymbol{k}\left[\mathbf{F}^{2 p}(\mathcal{W} \boldsymbol{g})\right] \subset \mathbf{F}^{p}\left(\Omega_{A}(M)\right), p \geq 0$. Moreover, if $\lambda$ is basic, then $\boldsymbol{k}\left[\mathbf{F}^{2 p}(\mathcal{W} \boldsymbol{g})\right] \subset \mathbf{F}^{2 p}\left(\Omega_{A}(M)\right), p \geq 0$.

Proof. Of course, it is sufficient to verify that $\boldsymbol{k}_{x}: W \boldsymbol{g}_{\mid x} \rightarrow \wedge A_{\mid x}^{*}$ preserves the filtrations. Since $I_{\mathbb{R} \otimes \bigvee{ }^{p} \boldsymbol{g}_{\mid x}^{*}}=\left(I_{\mathbb{R} \otimes \boldsymbol{g}_{\mid x}^{*}}\right)^{p}$, therefore $\mathbf{F}^{2 p}\left(W \boldsymbol{g}_{\mid x}\right)=\left(\mathbf{F}^{2}\left(\mathcal{W} \boldsymbol{g}_{\mid x}\right)\right)^{p}$. On the other hand, $\boldsymbol{k}_{x}$ is a homomorphism of algebras, thus we need only check the inclusion $\boldsymbol{k}_{x}\left[\mathbf{F}^{2}\left(W \boldsymbol{g}_{\mid x}\right)\right] \subset \mathbf{F}^{1}\left(\bigwedge A_{\mid x}^{*}\right)$, whereas, in the case of a basic connection $\boldsymbol{k}_{x}\left[\mathbf{F}^{2}\left(W \boldsymbol{g}_{\mid x}\right)\right] \subset$ $\mathbf{F}^{2}\left(\bigwedge A_{\mid x}^{*}\right)$.

$\mathbf{F}^{2}\left(W \boldsymbol{g}_{\mid x}\right), \mathbf{F}^{1}\left(\bigwedge A_{\mid x}^{*}\right)$ and $\mathbf{F}^{2}\left(\bigwedge A_{\mid x}^{*}\right)$ are ideals and $\mathbf{F}^{2}\left(W \boldsymbol{g}_{\mid x}\right)$ equals $I_{\mathbb{R}} \otimes g_{\mid x}^{*}$, so it suffices to check that $\boldsymbol{k}_{x}\left(1 \otimes w^{*}\right) \in I_{\bigwedge^{1}\left(A_{\mid x}^{\prime \perp}\right)}^{(2)}, w^{*} \in \boldsymbol{g}_{\mid x}^{*}$, and for a basic connection $\lambda$, that $\boldsymbol{k}_{x}\left(1 \otimes w^{*}\right) \in I_{\bigwedge^{2}\left(A_{\mid x}^{\prime \perp}\right)}^{(2)}, w^{*} \in \boldsymbol{g}_{\mid x}^{*}$.

However, $\boldsymbol{k}_{x}\left(1 \otimes w^{*}\right)=\left\langle w^{*}, \Omega_{\mid x}\right\rangle$, so the assertion follows from Theorem 9.4.

COROLlary 9.6. Let the situation be as in the previous proposition. If $q=\operatorname{rank}\left(E / E^{\prime}\right)$ (i.e. $q$ equals the codimension of $\mathcal{F}^{\prime}$ with respect to $\mathcal{F} ; \mathcal{F}^{\prime}$ and $\mathcal{F}$ being the foliations determined by $E$ and $E^{\prime}$, respectively), then

$$
\boldsymbol{k}\left[\mathbf{F}^{2 p}(\mathcal{W} \boldsymbol{g})\right]=0 \quad \text { for } \quad p \geq q+1 .
$$

If $\lambda$ is, in addition, basic, then

$$
\boldsymbol{k}\left[\mathbf{F}^{2 p}(\mathcal{W} \boldsymbol{g})\right]=0 \quad \text { for } \quad p \geq\left[\frac{q}{2}\right]+1 .
$$

Proof. Clearly, $q=\operatorname{rank}\left(A / A^{\prime}\right)=\operatorname{dim}\left(A_{\mid x}^{\prime \perp}\right)$ for each $x \in M$, which gives $\bigwedge^{p}\left(A_{\mid x}^{\prime \perp}\right)=0$ for $p \geq q+1$ and, in consequence, $\mathbf{F}^{p}(\Omega(A))=0$ for $p \geq q+1$; then Proposition 9.5 implies $\boldsymbol{k}\left[\mathbf{F}^{2 p}(\mathcal{W} \boldsymbol{g})\right]=0$ for such $p$. Under the additional assumption concerning $\lambda$, $\boldsymbol{k}\left[\mathbf{F}^{2 p}(\mathcal{W} \boldsymbol{g})\right]=0$ for $2 p \geq q+1$, i.e. for $p \geq\left[\frac{q}{2}\right]+1$.

The filtration of $\mathcal{W} \boldsymbol{g}$ in the intersection with the subalgebra $\bigoplus_{l \geq 0} \operatorname{Sec}\left(\bigvee^{l} \boldsymbol{g}^{*}\right)_{I^{0}}$ gives a filtration of the latter with

$$
\mathbf{F}^{2 p}\left(\bigoplus_{l \geq 0} \operatorname{Sec}\left(\bigvee^{l} \boldsymbol{g}^{*}\right)_{I^{0}}\right):=\left(\bigoplus_{l \geq 0} \operatorname{Sec}\left(\bigvee^{l} \boldsymbol{g}^{*}\right)_{I^{0}}\right) \cap \mathbf{F}^{2 p}(\mathcal{W} \boldsymbol{g})=\bigoplus_{l \geq p} \operatorname{Sec}\left(\bigvee^{l} \boldsymbol{g}^{*}\right)_{I^{0}}
$$

Notice also, see Corollary 9.3 and $\left[K 7\right.$, s. 2], that the isomorphism $\gamma_{*}: \Omega_{E}(M) \rightarrow \Omega_{A, i}(M)$ preserves the filtration. As a corollary we obtain the so-called "Vanishing Bott's Phenomenon" [K6] for regular Lie algebroids.

\section{The truncated Weil algebra}

DEFINITION 10.1. By the symmetric truncated algebra over a vector space $\mathfrak{g}$ we shall mean the space $\bigvee^{\leq l} \mathfrak{g}^{*}$ with the canonical even gradation, and with the structure of an (anti)commutative graded algebra such that

$$
\left(u_{1}^{*} \vee \ldots \vee u_{k}^{*}\right) \cdot\left(v_{1}^{*} \vee \ldots \vee v_{s}^{*}\right)=\left\{\begin{array}{rr}
u_{1}^{*} \vee \ldots \vee u_{k}^{*} \vee v_{1}^{*} \vee \ldots \vee v_{s}^{*} \text { when } k+s \leq l \\
0 & \text { when } k+s>l
\end{array}\right.
$$


This algebra can be constructed isomorphically as a quotient algebra $\left(\bigvee \mathfrak{g}^{*}\right) / I_{\bigvee^{>l}} \mathfrak{g}^{*}$ of the symmetric algebra $\bigvee \mathfrak{g}^{*}$ by the ideal generated by $\bigvee^{l+1} \mathfrak{g}^{*}$. The mapping

$$
\bigvee^{\leq l} \mathfrak{g}^{*} \rightarrow\left(\bigvee \mathfrak{g}^{*}\right) / I_{\bigvee^{>l} \mathfrak{g}^{*}}, \quad w^{*} \mapsto\left[w^{*}\right],
$$

establishes the canonical isomorphism of algebras. The canonical projection $\pi_{l}: \bigvee \mathfrak{g}^{*} \rightarrow$ $\bigvee^{\leq l} \mathfrak{g}^{*}$ is, of course, a homomorphism of algebras.

Denote by

$$
(W \mathfrak{g})_{l}:=\bigwedge \mathfrak{g}^{*} \otimes \bigvee^{\leq l} \mathfrak{g}^{*}
$$

the anticommutative graded tensor product of the anticommutative graded algebras. It is called the truncated Weil algebra of the vector space $\mathfrak{g}$.

We return to the consideration of a regular Lie algebroid $A$ over $(M, E)$, with the Atiyah sequence $0 \rightarrow \boldsymbol{g} \hookrightarrow A \stackrel{\gamma}{\rightarrow} E \rightarrow 0$. Notice that, for each $x \in M$,

$$
\left(W \boldsymbol{g}_{\mid x}\right)_{l} \cong\left(W \boldsymbol{g}_{\mid x}\right) / \mathbf{F}^{2(l+1)}\left(W \boldsymbol{g}_{\mid x}\right)
$$

$\left(\theta_{x} \mapsto\left[\theta_{x}\right]\right.$ establishes the canonical isomorphism) and, by the relation

$$
d_{x}\left[\left(W \boldsymbol{g}_{\mid x}\right)^{k, 2 s}\right] \subset\left(W \boldsymbol{g}_{\mid x}\right)^{k+1,2 s} \oplus\left(W \boldsymbol{g}_{\mid x}\right)^{k-1,2(s+1)},
$$

$d_{x}$ defines a new differential

$$
\left[d_{x}\right]_{l}:\left(W \boldsymbol{g}_{\mid x}\right)_{l} \rightarrow\left(W \boldsymbol{g}_{\mid x}\right)_{l} .
$$

Writing $d_{x}=d_{x}^{\prime}+d_{x}^{\prime \prime}$ where $d_{x}^{\prime}\left[\left(W \boldsymbol{g}_{\mid x}\right)^{k, 2 s}\right] \subset\left(W \boldsymbol{g}_{\mid x}\right)^{k+1,2 s}$ and $d_{x}^{\prime \prime}\left[\left(W \boldsymbol{g}_{\mid x}\right)^{k, 2 s}\right] \subset$ $\left(W \boldsymbol{g}_{\mid x}\right)^{k-1,2(s+1)}$, we see that

$$
\left[d_{x}\right]_{l}\left(\varphi_{x} \otimes \Gamma_{x}\right)=\left\{\begin{array}{l}
d_{x}\left(\varphi_{x} \otimes \Gamma_{x}\right) \text { when } \Gamma_{x} \in \bigvee^{<l} \boldsymbol{g}_{\mid x}^{*}, \\
d_{x}^{\prime}\left(\varphi_{x} \otimes \Gamma_{x}\right) \text { when } \Gamma_{x} \in \bigvee^{l} \boldsymbol{g}_{\mid x}^{*}
\end{array}\right.
$$

Put $(W \boldsymbol{g})_{l}:=\bigwedge \boldsymbol{g}^{*} \bigotimes \bigvee^{\leq l} \boldsymbol{g}^{*}$ and $(\mathcal{W} \boldsymbol{g})_{l}:=\operatorname{Sec}(W \boldsymbol{g})_{l}$. Of course,

$$
(W \boldsymbol{g})_{l} \cong(W \boldsymbol{g}) / \mathbf{F}^{2(l+1)}(W \boldsymbol{g}) .
$$

$(W \boldsymbol{g})_{l}$ will be called the truncated Weil algebra of the vector bundle $\boldsymbol{g}$.

The family $\left[d_{x}\right]_{l}, x \in M$, determines an endomorphism $[d]_{l}:(W \boldsymbol{g})_{l} \rightarrow(W \boldsymbol{g})_{l}$ and a differential, denoted by the same letter,

$$
[d]_{l}:(\mathcal{W} \boldsymbol{g})_{l} \rightarrow(\mathcal{W} \boldsymbol{g})_{l}
$$

For $s<l$, the projection $(\mathcal{W} \boldsymbol{g})_{l} \rightarrow(\mathcal{W g})_{s}$ is a homomorphism of algebras commuting with the differentials $[d]_{l}$ and $[d]_{s}$.

Take the canonical adjoint representation $\operatorname{ad}_{A}$ of $A$ on $(W \boldsymbol{g})_{l}$ and denote by $(\mathcal{W} \boldsymbol{g})_{l, I^{\circ}}$ the space (in fact, a subalgebra of $\left.(\mathcal{W} \boldsymbol{g})_{l}\right)$ of invariant cross-sections. $(\mathcal{W} \boldsymbol{g})_{l, I^{0}}$ is stable under the operator $[d]_{l}$. Indeed, let $\Theta$ be a bihomogeneous element of $(\mathcal{W} \boldsymbol{g})_{l, I^{0}}$. Then $d \Theta$ is invariant, in particular, $d^{\prime} \Theta$ is invariant; $[d]_{l} \Theta$ being equal to $d \Theta$ or $d^{\prime} \Theta$, is invariant, too.

Let $\lambda$ be any connection in $A$ and let $\boldsymbol{k}: \mathcal{W} \boldsymbol{g} \rightarrow \Omega_{A}(M)$ be the homomorphism of algebras determined by $\lambda$. 
Proposition 10.2. Assume that $\boldsymbol{k}\left[\mathbf{F}^{2(l+1)}(\mathcal{W} \boldsymbol{g})\right]=0$. Then

(1) there exists a homomorphism of algebras $[\boldsymbol{k}]_{l}:(\mathcal{W} \boldsymbol{g})_{l} \rightarrow \Omega_{A}(M)$ such that the diagram

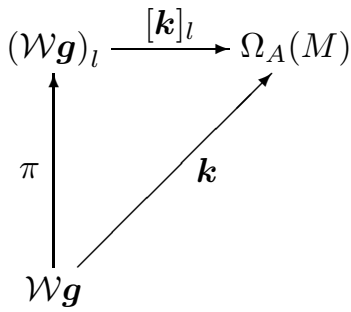

commutes ( $\pi$ being the canonical projection),

(2) $[\boldsymbol{k}]_{l}$ is equal to the restriction $\boldsymbol{k} \mid(\mathcal{W} \boldsymbol{g})_{l}$,

(3) $[\boldsymbol{k}]_{l}$ restricted to the invariant cross-sections $(\mathcal{W} \boldsymbol{g})_{l, I^{0}}$ commutes with the differentials $[d]_{l}$ and $d^{A}$, defining a homomorphism of algebras

$$
[\boldsymbol{k}]_{l \#}: H\left((\mathcal{W} \boldsymbol{g})_{l, I^{0}},[d]_{l}\right) \rightarrow H_{A}(M) .
$$

The class $[\boldsymbol{k}]_{l \#}[\Theta]$ for $\Theta \in\left(\operatorname{Sec} \bigwedge^{k} \boldsymbol{g}^{*} \otimes \bigvee^{s} \boldsymbol{g}^{*}\right)_{I^{0}}, s \leq l$, has the form $\frac{1}{k ! \cdot s !}$. $\langle\Theta, \underbrace{\omega \wedge \ldots \wedge \omega}_{k \text { times }} \otimes \underbrace{\Omega \vee \ldots \vee \Omega}_{s \text { times }}\rangle$ as its representative.

Proof. (1) and (2) are evident.

(3) Let $\Theta \in(\mathcal{W} \boldsymbol{g})_{l, I^{0}}\left(\subset(\mathcal{W} \boldsymbol{g})_{I^{0}}\right)$. By Lemma 7.1

$$
\begin{aligned}
d^{A} \circ[\boldsymbol{k}]_{l}(\Theta) & =d^{A} \circ \boldsymbol{k}(\Theta)=\boldsymbol{k} \circ d(\Theta)=[\boldsymbol{k}]_{l} \circ \pi \circ d(\Theta) \\
& =[\boldsymbol{k}]_{l} \circ[d]_{l} \circ \pi(\Theta)=[\boldsymbol{k}]_{l} \circ[d]_{l}(\Theta) .
\end{aligned}
$$

The last sentence is a consequence of Proposition 7.2 (see also [K7, Sec. 2]).

Example 10.3. Assume that $A$ is equipped with a flat partial connection $\lambda^{\prime}$ over $E^{\prime} \subset$ $E$ (as in Prop. 9.5) and let $q=\operatorname{rank}\left(E / E^{\prime}\right)$. According to Corollary 9.6, $\boldsymbol{k}\left[\mathbf{F}^{2(q+1)}(\mathcal{W} \boldsymbol{g})\right]$ $=0$ for an adapted connection $\lambda$, and $\boldsymbol{k}\left[\mathbf{F}^{2([q / 2]+1)}(\mathcal{W} \boldsymbol{g})\right]=0$ for a basic connection $\lambda$. Prop. 10.2 produces in these situations the homomorphisms of algebras $[\boldsymbol{k}]_{q^{\prime}}:(\mathcal{W} \boldsymbol{g})_{q^{\prime}} \rightarrow$ $\Omega_{A}(M)$ for $q^{\prime} \geq q$ and $q^{\prime} \geq[q / 2]$, respectively, and next, the corresponding homomorphisms on cohomology. The homomorphism $[\boldsymbol{k}]_{q \#}: H\left((\mathcal{W} \boldsymbol{g})_{q},[d]_{q}\right) \rightarrow H_{A}(M)$ generalizes the $\omega_{\#}$ described in [K7, Cor. 2.3 and Rem. 1]: In the case when $E^{\prime}=E$, i.e. when $\lambda^{\prime}$ is a flat connection in $A$, we have $q=0$ and $[\boldsymbol{k}]_{0}=\omega_{0}^{\wedge}$.

11. Characteristic homomorphism-construction. Here we construct some characteristic homomorphism of a partially flat regular Lie algebroid, which is a generalization of the one constructed in [K7] for a flat regular Lie algebroid.

Consider, in a given regular Lie algebroid $A$ over $(M, E)$, two geometric structures:

(1) a partial flat connection $\lambda^{\prime}$ over an involutive subdistribution $E^{\prime} \subset E$,

(2) a subalgebroid $B \subset A$ over $(M, E)$, see the following diagram: 


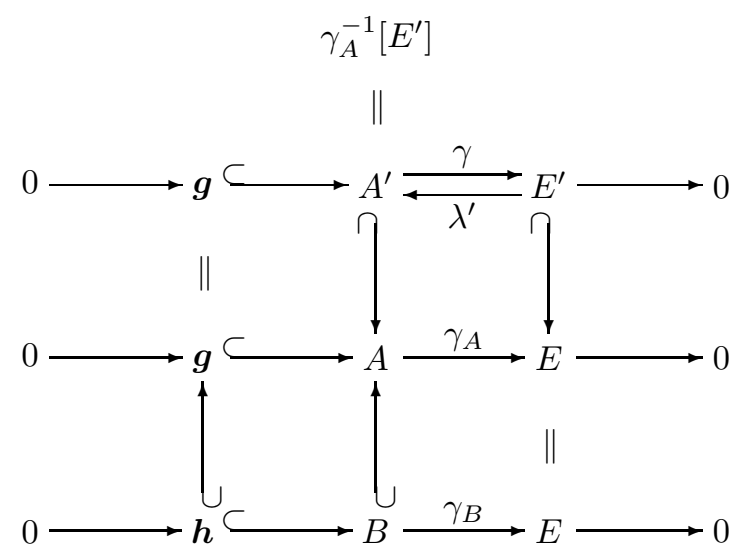

The system $\left(A, B, \lambda^{\prime}\right)$ is called a PFS-regular Lie algebroid over $\left(M, E, E^{\prime}\right)$.

The construction of the characteristic homomorphism of a PFS-regular Lie algebroid has, as in the case of an FS-regular Lie algebroid [K7], a number of steps.

1) Let $\mathbf{s}: \boldsymbol{g} \rightarrow \boldsymbol{g} / \boldsymbol{h}$ denote, as in [K7, Sec. 3], the canonical projection. Put, for a positive integer $l, W(\boldsymbol{g} ; \boldsymbol{h})_{l}:=\bigwedge(\boldsymbol{g} / \boldsymbol{h})^{*} \otimes \bigvee^{\leq l} \boldsymbol{g}^{*}$ and $\mathcal{W}(\boldsymbol{g} ; \boldsymbol{h})_{l}:=\operatorname{Sec} W(\boldsymbol{g} ; \boldsymbol{h})_{l} . \mathcal{W}(\boldsymbol{g} ; \boldsymbol{h})_{l}$ with the natural structure of an algebra will be called the truncated relative Weil algebra.

The representation $\operatorname{ad}_{B, \boldsymbol{g}}^{\wedge}$ of $B$ on $\bigwedge(\boldsymbol{g} / \boldsymbol{h})^{*}$ described in $[\mathrm{K} 7, \mathrm{p} .211]$, together with the representation $\operatorname{ad}_{A} \mid B$ of $B$ on $\bigvee^{\leq l} \boldsymbol{g}^{*}$ (the restriction to $B$ of the adjoint representation of $A$ on $\left.\bigvee^{\leq l} \boldsymbol{g}^{*}\right)$, yields the representation of $B$ on $W(\boldsymbol{g} ; \boldsymbol{h})_{l}$ denoted also - for brevity by ad. For an arbitrary $\xi \in \operatorname{Sec} B$, the differential operator $\mathcal{L}_{\text {ad } \circ \xi}: \mathcal{W}(\boldsymbol{g} ; \boldsymbol{h})_{l} \rightarrow \mathcal{W}(\boldsymbol{g} ; \boldsymbol{h})_{l}$ is a differentiation of the truncated relative Weil algebra $\mathcal{W}(\boldsymbol{g} ; \boldsymbol{h})_{l}$, from which we obtain that the space $\mathcal{W}(\boldsymbol{g} ; \boldsymbol{h})_{l, I^{0}}$ of invariant cross-sections is a subalgebra of $\mathcal{W}(\boldsymbol{g} ; \boldsymbol{h})_{l}$.

The monomorphisms $\bigwedge \mathbf{s}^{*}: \bigwedge(\boldsymbol{g} / \boldsymbol{h})^{*} \rightarrow \bigwedge \boldsymbol{g}^{*}$ and

$$
\bigwedge \mathbf{s}^{*} \otimes \operatorname{id}^{l}: \bigwedge(\boldsymbol{g} / \boldsymbol{h})^{*} \otimes \bigvee^{\leq l} \boldsymbol{g}^{*} \rightarrow \bigwedge \boldsymbol{g}^{*} \otimes \bigvee^{\leq l} \boldsymbol{g}^{*}
$$

of vector bundles are invariant with respect to the representations considered of the Lie algebroid $B$, which is easy to see by the definitions. As a corollary from the above we obtain that $\left(\bigwedge \mathbf{s}^{*} \otimes \mathrm{id}^{l}\right) \circ \Psi, \Psi \in \mathcal{W}(\boldsymbol{g} ; \boldsymbol{h})_{l}$, is an invariant cross-section if and only if $\Psi$ is invariant, and that

$$
\mathcal{W}(\boldsymbol{g} ; \boldsymbol{h})_{l, I^{0}} \ni \Psi \mapsto\left(\bigwedge \mathbf{s}^{*} \otimes \operatorname{id}^{l}\right) \circ \Psi \in \mathcal{W}(\boldsymbol{g})_{l, I^{0}}
$$

is a homomorphism of algebras. On the other hand, a cross-section $\Psi^{\prime}$ of $(\mathcal{W} \boldsymbol{g})_{l}$ is of the image of some cross-section of the bundle $W(\boldsymbol{g} ; \boldsymbol{h})_{l}$ if and only if $\Psi^{\prime}$ is $\boldsymbol{h}$-horizontal (i.e. if and only if $\iota_{\nu} \Psi=0$ for $\nu \in \operatorname{Sec} \boldsymbol{h}$, where $\iota_{\nu}$ is the operator defined in subsection 6), so

$$
\mathcal{W}(\boldsymbol{g} ; \boldsymbol{h})_{l, I^{0}} \rightarrow \mathcal{W}(\boldsymbol{g})_{l, \boldsymbol{h}, I^{0}}, \Psi \mapsto\left(\bigwedge \mathbf{s}^{*} \otimes \mathrm{id}^{l}\right) \circ \Psi,
$$

is an isomorphism of algebras where $\mathcal{W}(\boldsymbol{g})_{l, \boldsymbol{h}, I^{0}} \subset \mathcal{W}(\boldsymbol{g})_{l, I^{0}}$ is a subalgebra of $\boldsymbol{h}$-invariant elements.

2) The subspace $\mathcal{W}(\boldsymbol{g})_{l, \boldsymbol{h}, I^{0}}$ is stable under the differential (10.1). Indeed, for an invariant element $\Psi^{\prime}$ of $\mathcal{W}(\boldsymbol{g})_{l}$, we have $\iota_{\nu} \circ d\left(\Psi^{\prime}\right)=-d \circ \iota_{\nu}\left(\Psi^{\prime}\right)$ by Lemma 4.4 and Proposition 6.1, and, in consequence, $\iota_{\nu} \circ d^{\prime}\left(\Psi^{\prime}\right)=-d^{\prime} \circ \iota_{\nu}\left(\Psi^{\prime}\right)$. Therefore, for a bihomoge- 
neous element $\Psi^{\prime} \in \mathcal{W}(\boldsymbol{g})_{l}, \iota_{\nu} \circ[d]_{l}\left(\Psi^{\prime}\right)=\iota_{\nu}\left(d \Psi^{\prime}\right)=-d\left(\iota_{\nu} \Psi^{\prime}\right)=0$ or $\iota_{\nu} \circ[d]_{l}\left(\Psi^{\prime}\right)=$ $\iota_{\nu}\left(d^{\prime} \Psi^{\prime}\right)=-d^{\prime}\left(\iota_{\nu} \Psi^{\prime}\right)=0$, see Section 10.1. This enables us to define the differential $d_{l, \boldsymbol{h}}: \mathcal{W}(\boldsymbol{g} ; \boldsymbol{h})_{l, I^{0}} \rightarrow \mathcal{W}(\boldsymbol{g} ; \boldsymbol{h})_{l, I^{0}}$ in such a way that the following diagram commutes:

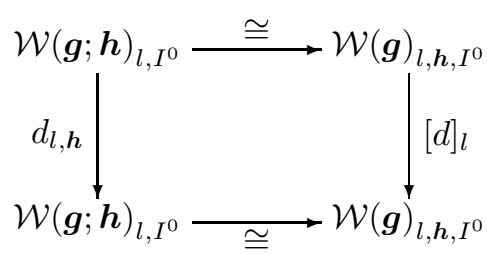

3) Consider any connection $\lambda: E \rightarrow A$ in $A$ and let the homomorphism $\mathbf{k}$ be constructed for $\lambda$. Assuming $\boldsymbol{k}\left[\mathbf{F}^{2(l+1)}(\mathcal{W} \boldsymbol{g})\right]=0$ (see Proposition 10.2) the form $\varphi(\Psi):=$ $[\mathbf{k}]_{l}\left(\left(\bigwedge \mathbf{s}^{*} \otimes \operatorname{id}^{l}\right) \circ \Psi\right), \Psi \in \mathcal{W}(\boldsymbol{g} ; \boldsymbol{h})_{l}$, is $\boldsymbol{h}$-horizontal, which follows in an easy way from Propositions 6.2 and 7.3. Therefore, the form $j^{*}(\varphi(\Psi)) \in \Omega_{B}(M)$ is horizontal. Then there exists a form $\Delta \Psi \in \Omega_{E}(M)$ such that

$$
\left(\gamma_{B}\right)_{*}(\Delta \Psi)=j^{*}\left([\mathbf{k}]_{l}\left(\left(\bigwedge \mathbf{s}^{*} \otimes \mathrm{id}^{l}\right) \circ \Psi\right)\right) .
$$

REMARK 11.1. One can easily check that if $\lambda$ is a connection in $B$, then for $\Psi \in$ $\operatorname{Sec} \bigwedge^{k}(\boldsymbol{g} / \boldsymbol{h})^{*} \otimes \bigvee^{l} \boldsymbol{g}^{*}$

$$
\Delta \Psi=\left\{\begin{array}{crl}
0 & \text { when } & k>0, \\
\lambda_{*}\left(\Omega^{\vee} \Psi\right) & \text { when } & k=0 .
\end{array}\right.
$$

4) Let $q=\operatorname{rank}\left(E / E^{\prime}\right)$ and let $\lambda$ be adapted to $\lambda^{\prime}$. Defined in the above manner, the mapping

$$
\Delta_{q^{\prime}}: \mathcal{W}(\boldsymbol{g} ; \boldsymbol{h})_{q^{\prime}} \rightarrow \Omega_{E}(M), \Psi \mapsto \Delta \Psi,
$$

$q^{\prime} \geq q$ (and $q^{\prime} \geq[q / 2]$ in the case of a basic connection), is a homomorphism of algebras, see Example 10.3 and the following commutative diagram.

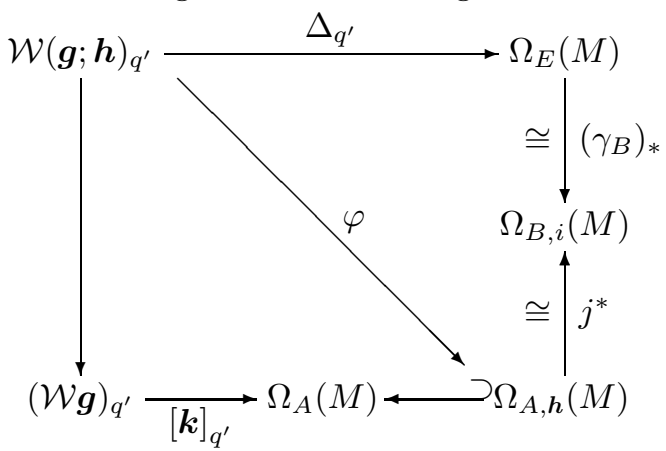

Proposition 11.2. The mapping $\Delta_{q^{\prime}}$ restricted to the invariant cross-sections

$$
\Delta_{q^{\prime} *}: \mathcal{W}(\boldsymbol{g} ; \boldsymbol{h})_{q^{\prime}, I^{0}} \rightarrow \Omega_{E}(M)
$$

commutes with the differentials $d_{q^{\prime}, h}$ and $d^{E}$.

Proof. $j$ and $\gamma_{B}$ are homomorphisms of regular Lie algebroids; then, according to the commutativity of $j^{*}$ with the differentials $d^{A}$ and $d^{B}$, and from the last diagram and the definition of $d_{l, \boldsymbol{h}}$, we notice that it is sufficient to show that $[\mathbf{k}]_{q^{\prime}}: \mathcal{W}(\boldsymbol{g})_{q^{\prime}, \boldsymbol{h}, I^{0}} \rightarrow \Omega_{A}(M)$ commutes with $[d]_{q^{\prime}}$ and $d^{A}$, but this follows from Proposition 10.2. 
As a corollary we obtain

THEOREM 11.3. The mapping

$$
\Delta_{q^{\prime} \#}: H\left(\mathcal{W}(\boldsymbol{g}, \boldsymbol{h})_{q^{\prime}, I^{0}}, d_{q^{\prime}, \boldsymbol{h}}\right) \rightarrow H_{E}(M), \quad[\Psi] \mapsto\left[\Delta_{q^{\prime} *} \Psi\right],
$$

is a correctly defined homomorphism of algebras.

$5)$ If $\lambda$ is basic, then the following diagram commutes:

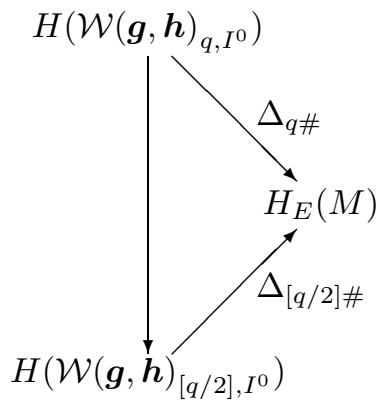

in which the vertical arrow is a homomorphism of algebras, induced by the projection.

$\Delta_{q \#}$ (also $\Delta_{[q / 2] \#}$ for a basic connection) is called the characteristic homomorphism of the PFS-regular Lie algebroid $\left(A, B, \lambda^{\prime}\right)$, its image is a subalgebra of $H_{E}(M)$ called the characteristic algebra of the PFS-regular Lie algebroid $\left(A, B, \lambda^{\prime}\right)$, and its elements the characteristic classes of this algebroid.

12. The functoriality and other properties. Let $\left(A_{i}, B_{i}, \lambda_{i}^{\prime}\right)$ be PFS-regular Lie algebroids over $\left(M_{i}, E_{i}, E_{i}^{\prime}\right), i=1,2$.

Definition 12.1. By a homomorphism $H:\left(A_{1}, B_{1}, \lambda_{1}^{\prime}\right) \rightarrow\left(A_{2}, B_{2}, \lambda_{2}^{\prime}\right)$ we mean a homomorphism $H: A_{1} \rightarrow A_{2}$ of regular Lie algebroids, say over $f:\left(M_{1}, E_{1}\right) \rightarrow\left(M_{2}, E_{2}\right)$ such that (1) $f_{*}\left[E_{1}^{\prime}\right] \subset E_{2}^{\prime},(2) H \circ \lambda_{1}^{\prime}=\lambda_{2}^{\prime} \circ f_{*} \mid E_{1}^{\prime}$, (3) $H\left[B_{1}\right] \subset B_{2}$.

In the sequel $\boldsymbol{g}_{i}, \boldsymbol{h}_{i}, A_{i}^{\prime}, E_{i}^{\prime}$ denote the analogous objects related to $A_{i}, B_{i}$ and $E_{i}$ as in diagram (11) for $\boldsymbol{g}, \boldsymbol{h}, A$ and $E$, respectively.

By the pullback of a PFS-regular Lie algebroid $\left(A, B, \lambda^{\prime}\right)$ over $\left(M, E, E^{\prime}\right)$ via a mapping $f:\left(M_{1}, E_{1}, E_{1}^{\prime}\right) \rightarrow\left(M, E, E^{\prime}\right)$, i.e. a smooth mapping $f: M_{1} \rightarrow M$ such that $f_{*}\left[E_{1}\right] \subset E$ and $f_{*}\left[E_{1}^{\prime}\right] \subset E^{\prime}$, we mean the PFS-regular Lie algebroid $\left(f^{\wedge} A, f^{\wedge} B, \bar{\lambda}^{\prime}\right)$ where $\bar{\lambda}^{\prime}: E_{1}^{\prime} \rightarrow f^{\wedge} A_{1}$ is the pullback of $\lambda^{\prime}[\mathrm{K} 2,3.2 .1]: \bar{\lambda}^{\prime}(v)=\left(v, \lambda^{\prime}\left(f_{*} v\right)\right), v \in E_{1}^{\prime}$. Proposition [K2, Prop. 3.3.2] gives the flatness of $\bar{\lambda}^{\prime}$. The canonical homomorphism $\mathrm{pr}_{2}: f^{\wedge} A \rightarrow A$ is a homomorphism of PFS-regular Lie algebroids.

Let $H: A_{1} \rightarrow A_{2}$ be a homomorphism of regular Lie algebroids over $f:\left(M_{1}, E_{1}\right) \rightarrow$ $\left(M_{2}, E_{2}\right)$. Define the pullback

$$
H^{+*}: \mathcal{W}\left(\boldsymbol{g}_{2}\right) \rightarrow \mathcal{W}\left(\boldsymbol{g}_{1}\right)
$$

in the standard way: $H^{+*}(\Psi)(x)=H_{x}^{+*}\left(\Psi_{f(x)}\right), x \in M$, where $H_{x}^{+*}=\bigwedge H_{\mid x}^{+*} \otimes \bigvee H_{\mid x}^{+*}$. It is clear that $H^{+*}$ is a homomorphism of algebras of bidegree $(0,0)$.

Proposition 12.2. The pullback $H^{+*}$ has the following properties:

(1) $i_{x, v} \circ H_{x}^{+*}=H_{x}^{+*} \circ i_{f(x), H^{+}(v)}$ for $v \in \boldsymbol{g}_{1 \mid x}, x \in M_{1}$; in consequence, $H^{+*}$ maps $\boldsymbol{h}_{2}$-horizontal elements into $\boldsymbol{h}_{1}$-horizontal ones. 
(2) $\delta \circ H^{+*}(\Theta)=H^{+*} \circ \delta(\Theta)$ for $\Theta \in \operatorname{Sec}\left(\bigwedge \boldsymbol{g}_{2}^{*}\right)$, where $\delta$ 's denote the differentials [K' (2.5)].

(3) $\varphi \circ H^{+*}=H^{+*} \circ \varphi$ where $\varphi^{\prime}$ s denote the change of variable, see Subsec. 5.1.

(4) $d \circ H^{+*}=H^{+*} \circ d$,

(5) $[d]_{l_{1}} \circ H^{+*}=H^{+*} \circ[d]_{l_{2}}$ for $l_{1} \leq l_{2}$.

(6) $\mathcal{L}_{\mathrm{ad}(v)} \circ H_{x}^{+*}=H_{x}^{+*} \circ \mathcal{L}_{\mathrm{ad}(H v)}$ for $v \in A_{1 \mid x}$; in consequence, $H^{+*}$ maps invariant elements into invariant ones.

Proof. (1) Since $H_{x}^{+*}$ is a homomorphism and $i_{x, v}$ is an antiderivation, it is sufficient to check the equality for the elements of $W\left(\boldsymbol{g}_{2 \mid f(x)}\right)$ of the forms $\theta \otimes 1$ and $1 \otimes \theta$, where $\theta \in \boldsymbol{g}_{2 \mid f(x)}^{*}$.

$$
\begin{aligned}
i_{x, v} \circ H_{x}^{+*}(\theta \otimes 1) & =i_{x, v}\left(H_{x}^{+*} \theta \otimes 1\right)=i_{x, v}\left(H_{x}^{+*} \theta\right)=\left\langle\theta, H^{+}(v)\right\rangle \\
& =i_{f(x), H^{+}(v)}(\theta)=H_{x}^{+*} \circ i_{f(x), H^{+}(v)}(\theta \otimes 1) . \\
i_{x, v} \circ H_{x}^{+*}(1 \otimes \theta) & =i_{x, v}\left(1 \otimes H_{x}^{+*} \theta\right)=H_{x}^{+*} \circ i_{f(x), H^{+}(v)}(1 \otimes \theta) .
\end{aligned}
$$

(2) $\delta$ 's are antiderivations, therefore it is sufficient to consider $\Theta \in \operatorname{Sec} \boldsymbol{g}_{2}^{*}$. For $x \in M_{1}$ and $w_{i} \in \boldsymbol{g}_{1 \mid x}$

$$
\begin{aligned}
& \left\langle\left(\delta \circ H^{+*} \Theta\right)_{x}, w_{0} \wedge w_{1}\right\rangle=\left\langle\Theta_{f(x)}, H_{x}^{+}\left[w_{0}, w_{1}\right]\right\rangle \\
& =\left\langle(\delta \Theta)_{f(x)}, H_{x}^{+}\left(w_{0}\right) \wedge H_{x}^{+}\left(w_{1}\right)\right\rangle=\left\langle\left(H^{+*} \circ \delta(\Theta)\right)_{x}, w_{0} \wedge w_{1}\right\rangle .
\end{aligned}
$$

(3) By (2) above we easily check the equality on generators $1, \Theta \otimes 1,1 \otimes \Theta$.

(4) Thanks to the previous property, it follows from the equality $\bar{d} \circ H^{+*}=H^{+*} \circ \bar{d}$ which can be checked trivially.

(5) Property (4) yields $d^{\prime} \circ H^{+*}=H^{+*} \circ d^{\prime}$ and then (5) follows immediately.

(6) First, we show, for $\Theta \in \operatorname{Sec} g_{2}^{*}$, that

$$
\left\langle\mathcal{L}_{\mathrm{ad}^{\natural} \circ \xi_{1}}\left(H^{+*} \Theta\right), \nu_{1}\right\rangle=\left\langle H^{+*}\left(\mathcal{L}_{\mathrm{ad}^{\natural} \circ \xi_{2}} \Theta\right), \nu_{1}\right\rangle
$$

where $H \circ \xi_{1}=\xi_{2} \circ f$ and $\nu_{1} \in \operatorname{Sec} \boldsymbol{g}_{1}$ is a cross-section for which there exists $\nu_{2} \in \operatorname{Sec} \boldsymbol{g}_{2}$ fulfilling $H^{+} \circ \nu_{1}=\nu_{2} \circ f$. For this purpose, we notice [K2] that $H^{+} \circ \llbracket \xi_{1}, \nu_{1} \rrbracket=\llbracket \xi_{2}, \nu_{2} \rrbracket \circ f$. Thus

$$
\begin{aligned}
& \left\langle\mathcal{L}_{\mathrm{ad}^{\natural} \circ \xi_{1}}\left(H^{+*} \Theta\right), \nu_{1}\right\rangle=\left(\gamma_{1} \circ \xi_{1}\right) \circ\left\langle H^{+*} \Theta, \nu_{1}\right\rangle-\left\langle H^{+*} \Theta, \llbracket \xi_{1}, \nu_{1} \rrbracket\right\rangle \\
& =\left(\gamma_{2} \circ \xi_{2}\right)\left\langle\Theta, \nu_{2}\right\rangle \circ f-\left\langle\Theta, \llbracket \xi_{2}, \nu_{2} \rrbracket\right\rangle \circ f=\left\langle\mathcal{L}_{\mathrm{ad}^{\natural} \circ \xi_{2}} \Theta, \nu_{2}\right\rangle \circ f \\
& =\left\langle H^{+*}\left(\mathcal{L}_{\mathrm{ad}^{\natural} \circ \xi_{2}} \Theta\right), \nu_{1}\right\rangle .
\end{aligned}
$$

Lemma 4.4 leads now to the equality

$$
\left\langle\mathcal{L}_{\mathrm{ad} \circ \xi_{1}}\left(H^{+*} \Psi\right), \nu_{1}\right\rangle=\left\langle H^{+*}\left(\mathcal{L}_{\mathrm{ad} \circ \xi_{2}} \Psi\right), \nu_{1}\right\rangle,
$$

$\Psi \in \mathcal{W} \boldsymbol{g}_{2}$, where $\nu_{1}, \xi_{i}$ are as above.

The equality $\mathcal{L}_{\mathrm{ad} \circ \xi_{1}}\left(H^{+*} \Psi\right)=H^{+*}\left(\mathcal{L}_{\mathrm{ad} \circ \xi_{2}} \Psi\right)$ follows in an evident manner from those for a strong homomorphism and for a canonical one. In each of these cases, this follows from (12.1) and the observation that

- for arbitrary $x \in M_{1}$ and $v \in \boldsymbol{g}_{1 \mid x}$, there exist local cross-sections $\nu_{1}$ and $\nu_{2}$ such that $\nu_{1}(x)=v$ and $\nu_{1}$ and $\nu_{2}$ fulfil the required condition $H^{+} \circ \nu_{1}=\nu_{2} \circ f$.

Hence we obtain (6). 
COROLlary 12.3. $H^{+*}$ maps $\boldsymbol{h}_{2}$-horizontal and invariant elements into $\boldsymbol{h}_{1}$-horizontal and invariant ones, defining, for $l_{2} \geq l_{1}$, a homomorphism of algebras

$$
H^{+*}:\left(\mathcal{W} g_{2}\right)_{l_{2}, h_{2}, I^{0}} \rightarrow\left(\mathcal{W} \boldsymbol{g}_{1}\right)_{l_{1}, h_{1}, I^{0}}
$$

commuting with the differentials (i.e. $[d]_{l_{1}} \circ H^{+*}=H^{+*} \circ[d]_{l_{2}}$ ).

Each homomorphism $H:\left(A_{1}, B_{1}, \lambda_{1}^{\prime}\right) \rightarrow\left(A_{2}, B_{2}, \lambda_{2}^{\prime}\right)$ of PFS-regular Lie algebroids can be represented in the form of a superposition of a strong homomorphism with the canonical one

$$
\left(A_{1}, B_{1}, \lambda_{1}^{\prime}\right) \stackrel{\bar{H}}{\rightarrow}\left(f^{\wedge} A_{2}, f^{\wedge} B_{2}, \bar{\lambda}_{2}^{\prime}\right) \stackrel{\mathrm{pr}_{2}}{\rightarrow}\left(A_{2}, B_{2}, \lambda_{2}^{\prime}\right) .
$$

In the standard way, one can define the pullback

$$
[H]^{+*}: \mathcal{W}\left(\boldsymbol{g}_{2}, \boldsymbol{h}_{2}\right) \rightarrow \mathcal{W}\left(\boldsymbol{g}_{1}, \boldsymbol{h}_{1}\right)
$$

$\left([H]^{+*}(\Psi)(x)=\bigwedge\left[H_{x}^{+}\right]^{*} \otimes \bigvee H_{x}^{*}(\Psi(x))\right.$ where $\left[H_{x}^{+}\right]^{*}: \boldsymbol{g}_{1} / \boldsymbol{h}_{1} \rightarrow \boldsymbol{g}_{2} / \boldsymbol{h}_{2}$ is the induced linear homomorphism). Since the following diagram (for $l_{2} \geq l_{1}$ )

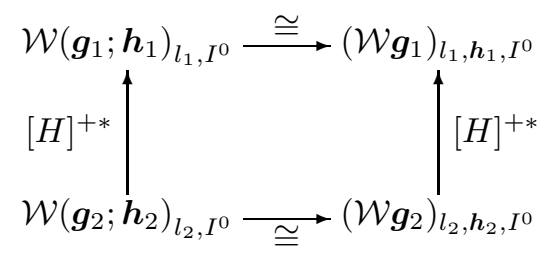

commutes, we obtain by the above that $[H]^{+*}$ commutes with the differentials, i.e. $H^{+*} \circ$ $[d]_{l_{2}, h_{2}}=[d]_{l_{1}, h_{1}} \circ[H]^{+*}$ giving a homomorphism on cohomology

$$
[H]^{+\#}: H\left(\mathcal{W}\left(\boldsymbol{g}_{2}, \boldsymbol{h}_{2}\right)_{l_{2}, I^{0}}\right) \rightarrow H\left(\mathcal{W}\left(\boldsymbol{g}_{1}, \boldsymbol{h}_{1}\right)_{l_{1}, I^{0}}\right) .
$$

THEOREM 12.4 (Functoriality of $\left.\Delta_{q \#}\right)$. Let $\left(A_{1}, B_{1}, \lambda_{1}^{\prime}\right)$ and $\left(A_{2}, B_{2}, \lambda_{2}^{\prime}\right)$ be PFS-regular Lie algebroids over $\left(M_{1}, E_{1}, E_{1}^{\prime}\right)$ and $\left(M_{2}, E_{2}, E_{2}^{\prime}\right)$, respectively; put $q_{i}=\operatorname{rank}\left(E_{i} / E_{i}^{\prime}\right)$. Let $H:\left(A_{1}, B_{1}, \lambda_{1}^{\prime}\right) \rightarrow\left(A_{2}, B_{2}, \lambda_{2}^{\prime}\right)$ be a homomorphism over $f:\left(M_{1}, E_{1}, E_{1}^{\prime}\right) \rightarrow$ $\left(M_{2}, E_{2}, E_{2}^{\prime}\right)$. Assume that the adapted connections $\lambda_{1}$ and $\lambda_{2}$, such that $H \circ \lambda_{1}=\lambda_{2} \circ f_{*}$, are given. Then the following diagram commutes:

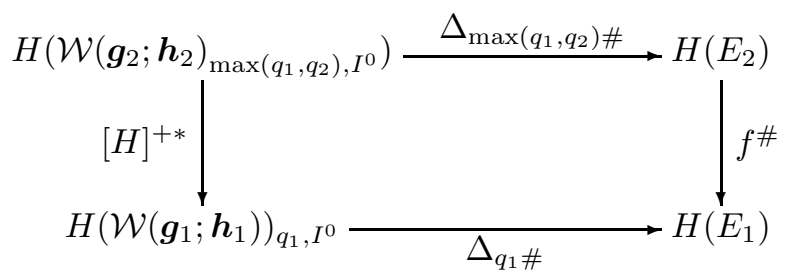

Proof. From the commutativity of diagrams (11.2) and (11.3) it follows that it is sufficient to check the same for the diagram

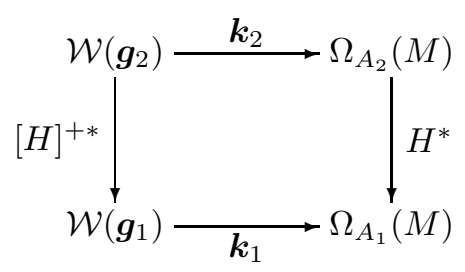


in which $\boldsymbol{k}_{2}$ and $\boldsymbol{k}_{1}$ are defined for $\lambda_{2}$ and $\lambda_{1}$, respectively. Using (a) $H_{*}^{+} \omega_{1}=H^{+*} \omega_{2}$ and (b) $H_{*}^{+} \Omega_{1}=H^{*} \Omega_{2}\left(\omega_{i}\right.$ and $\Omega_{i}$ being the connection forms and the curvature forms of $\left.\lambda_{i}, i=1,2\right)$, one can prove the commutativity at each point $x \in M$, considering the generators $1,1 \otimes \theta, \theta \otimes 1, \theta \in \boldsymbol{g}_{2 \mid f(x)}^{*}$, only. Equality (a) is evident, whereas (b) follows from $[\mathrm{K} 2,3.2 .2]$ and the horizontality of the curvature forms.

Theorem 12.5 (Independence of $\Delta_{q^{\prime} \#}$ of an adapted connection). For any PFSregular Lie algebroid $\left(A, B, \lambda^{\prime}\right)$ over $\left(M, E, E^{\prime}\right)$, the characteristic homomorphism 11.4 for $q^{\prime} \geq \operatorname{rank}\left(E / E^{\prime}\right)$ is independent of the choice of an adapted connection.

Proof. Let us consider any two connections $\lambda_{0}, \lambda_{1}: E \rightarrow A$ adapted to $\lambda^{\prime}$ and the connection $\lambda: T \mathbb{R} \times E \rightarrow T \mathbb{R} \times A$ in $T \mathbb{R} \times A$ defined by

$$
\lambda_{\mid(t, x)}(v, w)=\left(v, \lambda_{0}(w) \cdot(1-t)+\lambda_{1}(w) \cdot t\right), \quad(v, w) \in T_{t} \mathbb{R} \times E_{\mid x} .
$$

$\lambda$ is adapted to the flat partial connection id $\times \lambda^{\prime}: T \mathbb{R} \times E^{\prime} \rightarrow T \mathbb{R} \times A^{\prime}$. Of course, the system $\left(T \mathbb{R} \times A, T \mathbb{R} \times B\right.$, id $\left.\times \lambda^{\prime}\right)$ is a PFS-regular Lie algebroid and $\lambda$ is an adapted connection. One can prove that the connection form $\omega: T \mathbb{R} \times A \rightarrow 0 \times \boldsymbol{g}$ of $\lambda$ equals $\omega_{(t, x)}(v, w)=\left(0, \omega_{0}(w) \cdot(1-t)+\omega_{1}(w) \cdot t\right),(v, w) \in T_{t} \mathbb{R} \times A_{\mid x}$, where $\omega_{0}$ and $\omega_{1}$ are the connection forms of $\lambda_{0}$ and $\lambda_{1}$, respectively. The homomorphisms $F_{i}: A \rightarrow T \mathbb{R} \times A$, $w \mapsto\left(\theta_{i}, w\right), i=0,1$, of regular Lie algebroids (over $f_{i}: M \rightarrow \mathbb{R} \times M, x \mapsto(i, x)$ ), defined in [K7, Sec. 5], give homomorphisms $F_{i}:\left(A, B, \lambda^{\prime}\right) \rightarrow\left(T \mathbb{R} \times A, T \mathbb{R} \times B\right.$, id $\left.\times \lambda^{\prime}\right)$ of PFS-regular Lie algebroids such that $F_{i} \circ \lambda_{i}=\lambda \circ f_{i *}, i=0,1$. The principle of functoriality (Theorem 12.4) ensures the commutativity of the diagrams

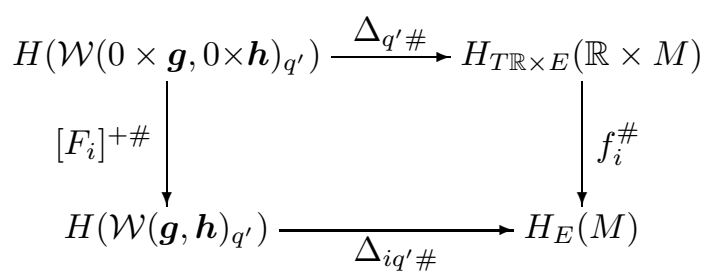

$i=0,1$. Since $f_{0}^{\#}=f_{1}^{\#}$ (see the proof of Th. 4.3 .1 from [K2]) and the superposition $A \stackrel{F_{3}}{\rightarrow} T \mathbb{R} \times A \stackrel{\mathrm{pr}_{2}}{\rightarrow} A$ of homomorphisms of regular Lie algebroids is equal to $\mathrm{id}_{A}$, this gives $\left[F_{i}\right]^{+\#} \circ\left[\mathrm{pr}_{2}\right]^{+\#}=\mathrm{id}\left(\mathrm{pr}_{2}\right.$ does not determine a PFS-homomorphism, but this is no problem), therefore we have

$$
\begin{aligned}
\Delta_{0 q^{\prime} \#} & =\Delta_{0 q^{\prime} \#} \circ\left[F_{0}\right]^{+\#} \circ\left[\mathrm{pr}_{2}\right]^{+\#}=f_{0}^{\#} \circ \Delta_{0 q^{\prime} \#} \circ\left[\mathrm{pr}_{2}\right]^{+\#} \\
& =f_{1}^{\#} \circ \Delta_{0 q^{\prime} \#} \circ\left[\mathrm{pr}_{2}\right]^{+\#}=\Delta_{1 q^{\prime} \#} \circ\left[F_{1}\right]^{+\#} \circ\left[\mathrm{pr}_{2}\right]^{+\#}=\Delta_{1 q^{\prime} \# .}
\end{aligned}
$$

Definition 12.6. Let us consider two PFS-regular Lie algebroids $\left(A, B_{i}, \lambda^{\prime}\right), i=0,1$ (which differ only in subalgebroids) over $\left(M, E, E^{\prime}\right)$. By analogy with definition $[\mathrm{K} 7$, Def. 5.4] we say that the characteristic homomorphisms $\Delta_{i q^{\prime} \#}: H\left(\mathcal{W}\left(\boldsymbol{g}, \boldsymbol{h}_{i}\right)_{q^{\prime}, I^{0}}\right) \rightarrow$ $H_{E}(M), i=0,1, q^{\prime} \geq \operatorname{rank}\left(E / E^{\prime}\right)$, are equivalent if there exists an isomorphism of algebras $\alpha: H\left(\mathcal{W}\left(\boldsymbol{g}, \boldsymbol{h}_{0}\right)_{q^{\prime}, I^{0}}\right) \rightarrow H\left(\mathcal{W}\left(\boldsymbol{g}, \boldsymbol{h}_{1}\right)_{q^{\prime}, I^{0}}\right)$ such that $\Delta_{0 q^{\prime} \#}=\Delta_{1 q^{\prime} \#} \circ \alpha$.

THEOREM 12.7 (Homotopic independence). If $B_{0}$ and $B_{1}$ are homotopic (for definition, see [K', Def. 5.1]), then $\Delta_{0 q^{\prime} \#}$ and $\Delta_{1 q^{\prime} \#}$ are equivalent. 
Proof. By the same argument as in the proof of [K7, Prop. 5.3], we see that $\Delta_{0 q^{\prime}} \#$ and $\Delta_{1 q^{\prime} \#}$ are related via the commutative diagram:

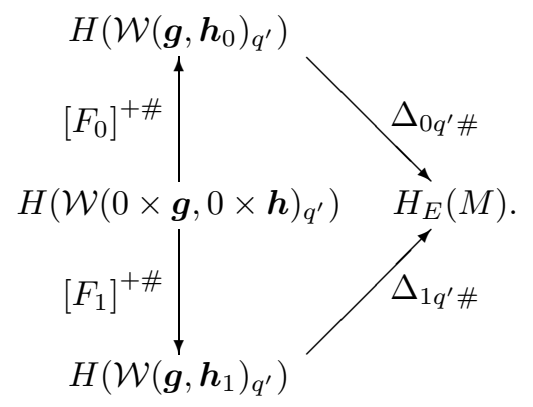

It remains to show that $\left[F_{i}\right]^{+\#}$ is an isomorphism of algebras, $i=0,1$. We do it as in the proof of $\left[\mathrm{K} 7\right.$, Th. 5.5]. For $F_{i}$ being the superposition $\mathrm{pr}_{2} \circ \bar{F}_{i}$ (in which $\bar{F}_{i}$ is an isomorphism), the problem reduces to the consideration of the canonical projection $\operatorname{pr}_{2}:\left(f_{i}^{\wedge}(T \mathbb{R} \times A), f_{i}^{\wedge} B, \overline{\mathrm{id} \times \lambda^{\prime}}\right) \rightarrow\left(T \mathbb{R} \times A, B, \mathrm{id} \times \lambda^{\prime}\right)$, more exactly, to the investigation of the homomorphism

$$
\mathrm{pr}_{2}^{+*}: \mathcal{W}(0 \times \boldsymbol{g}, 0 \times \boldsymbol{h})_{q^{\prime}, I^{0}} \rightarrow \mathcal{W}\left(f_{i}^{*}(0 \times \boldsymbol{g}), f_{i}^{*}(0 \times \boldsymbol{h})\right)_{q^{\prime}, I^{0}} .
$$

After the canonical identification

$$
f_{i}^{*}\left(\bigwedge(0 \times \boldsymbol{g} / \boldsymbol{h})^{*} \otimes \bigvee^{\leq q^{\prime}}(0 \times \boldsymbol{g})^{*}\right) \cong \bigwedge\left(f_{i}^{*}(0 \times \boldsymbol{g}) / f_{i}^{*}(0 \times \boldsymbol{h})\right)^{*} \otimes \bigvee^{\leq q^{\prime}} f_{i}^{*}(0 \times \boldsymbol{g})^{*},
$$

according to $f_{i}^{*}\left(\operatorname{ad}_{B, \boldsymbol{g}}\right)=\operatorname{ad}_{f_{i}^{\wedge} B, f_{i}^{*} g}[\mathrm{~K} 7]$ and the fact that $f_{i}^{*}\left(\otimes^{k} T\right)=\otimes^{k}\left(f_{i}^{*} T\right)$ for any representation $T$ (cf. $[\mathrm{K} 2,2.3 .3]$ and the proof of $[\mathrm{K} 7$, Prop. 4.2]), we obtain that $f_{i}^{\wedge}(\mathrm{ad})=$ ad (the ad's denote the canonical representations induced by the adjoint one), and that $\operatorname{pr}_{2}^{+*} \Psi=f_{i}^{*} \Psi$. As in the proof of $[K 7$, Th. 5.5], the rest follows from Theorem 20.2 below.

13. A comparison with the tangential classes of partially flat principal bundles. A PFS-regular Lie algebroid $\left(A, B, \lambda^{\prime}\right)$ over $\left(M, E, E^{\prime}\right)$ determines an FS-regular Lie algebroid $\left(A^{\prime}, B^{\prime}, \lambda^{\prime}\right)$ over $\left(M, E^{\prime}\right)$ in which $A^{\prime}=\gamma_{A}^{-1}\left[E^{\prime}\right], B^{\prime}=\gamma_{B}^{-1}\left[E^{\prime}\right]$. With these objects we have associated some homomorphisms: $\Delta_{q^{\prime} *}: \mathcal{W}(\boldsymbol{g}, \boldsymbol{h})_{q^{\prime}, I_{B}^{0}} \rightarrow \Omega_{E}(M)$ and $\Delta_{*}: \mathcal{W}(\boldsymbol{g}, \boldsymbol{h})_{0, I_{B^{\prime}}^{0}} \rightarrow \Omega_{E^{\prime}}(M)$ (see Theorem 11.3 and $[\mathrm{K} 7$, Prop. 3.3]). The indices $B$ and $B^{\prime}$ on $I$ indicate the regular Lie algebroid with respect to which the invariant elements are taken. A simple relation between $\Delta_{q^{\prime} *}$ and $\Delta_{*}$ is described by the following diagram:

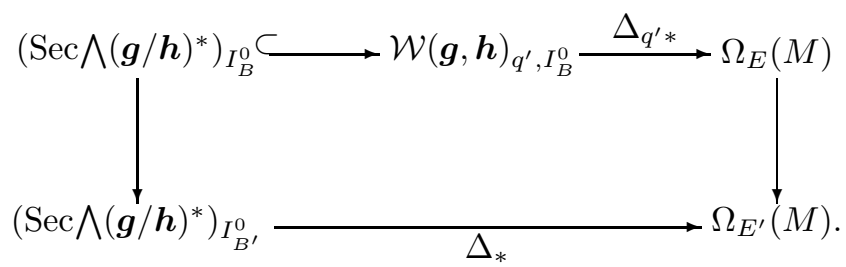

REMARK 13.1. The problem of what the relation looks like on the cohomology level will not be investigated here. We only notice that each element $\Psi \in\left(\operatorname{Sec} \bigwedge(\boldsymbol{g} / \boldsymbol{h})^{*}\right)_{I_{B}^{0}}$ 
being a cycle in $\mathcal{W}(\boldsymbol{g}, \boldsymbol{h})_{q^{\prime}, I_{B}^{0}}$ (i.e. with respect to $\left.[d]_{q^{\prime}}\right)$ is a cycle in $\mathcal{W}(\boldsymbol{g}, \boldsymbol{h})_{0}$ (i.e. with respect to $\bar{\delta}$ ); the converse is not true in general, which may be the source of the characteristic classes (in $H_{E^{\prime}}(M)$ ) measuring the concordance of $\lambda^{\prime}$ with $B$, which cannot be obtained by $\Delta_{q^{\prime}}$.

14. A comparison with the characteristic classes of foliated bundles. Let there be given:

(a) a $G$-principal fibre bundle $P=(P, \pi, M, G, \cdot)$,

(b) a flat partial connection in $P$ over an involutive regular distribution $F \subset T M$,

(c) a closed Lie subgroup $H \subset G$ and an $H$-reduction $P^{\prime} \subset P$.

In other words, we are given some foliated principal bundle with a reduction, considered, for example, in [K-T2]. As usual, let $\mathfrak{g}$ and $\mathfrak{h}$ denote the Lie algebras of $G$ and $H$, respectively. In [K-T2], to such a bundle there corresponds a characteristic homomorphism $\hat{\Delta}_{1 q^{\prime} \#}: H(\mathfrak{g}, H)_{q^{\prime}} \rightarrow H_{d R}(M)$ (denoted there by $\Delta$ ) where $q^{\prime} \geq \operatorname{codim} \mathcal{F}, \mathcal{F}$ being the foliation determined by $F$, and

$$
H(\mathfrak{g}, H)_{q^{\prime}}=\left(\bigwedge \mathfrak{g}^{*} \otimes \bigvee^{\leq q^{\prime}} \mathfrak{g}^{*}\right)_{H} \stackrel{S}{\cong}\left(\bigwedge(\mathfrak{g} / \mathfrak{h})^{*} \otimes \bigvee^{\leq q^{\prime}} \mathfrak{g}^{*}\right)_{I_{H}}
$$

is the truncated relative Weil algebra constructed isomorphically as the subalgebra of the truncated algebra $\bigwedge(\mathfrak{g} / \mathfrak{h})^{*} \otimes \bigvee^{\leq q^{\prime}} \mathfrak{g}^{*}$, consisting only of those elements which are invariant with respect to the representation $\mathrm{Ad}^{q^{\prime}}$ of $H$ induced by the restriction to $H$ of the adjoint representation $\operatorname{Ad}_{G}: G \rightarrow G L(\mathfrak{g})$. The differential $d_{q^{\prime}}$ in $W(\mathfrak{g}, H)_{q^{\prime}}$, defined in the standard way, comes from the differential, denoted here by $d_{\mathfrak{g}}^{L}$, in the Weil algebra $W \mathfrak{g}=\bigwedge \mathfrak{g}^{*} \otimes \bigvee \mathfrak{g}^{*}$, defined as follows: we treat $\mathfrak{g}$ as a left Lie algebra of $G$ (with the bracket denoted by $\left.[\cdot, \cdot]^{L}\right)$ and $d_{\mathfrak{g}}^{L}: W \mathfrak{g} \rightarrow W \mathfrak{g}$ is an antiderivation of total degree +1 such that $d_{\mathfrak{g}}^{L}\left(w^{*} \otimes 1\right)=1 \otimes w^{*}+d_{\wedge} w^{*} \otimes 1$ and $\iota_{v} d_{\mathfrak{g}}^{L}\left(1 \otimes w^{*}\right)=\theta_{\mathfrak{g}}^{L} w^{*}$ for $v \in \mathfrak{g}, w^{*} \in \mathfrak{g}^{*}\left(d_{\wedge}\right.$ is the Chevalley-Eilenberg differential, whereas $\theta_{\mathfrak{g}}^{L} w^{*}=-w^{*} \circ \operatorname{ad}_{\mathfrak{g}}^{L}$ where $\operatorname{ad}_{\mathfrak{g}}^{L}(\mu)=[\cdot, \mu]^{L}$, $\mu \in \mathfrak{g})$. In the sequel, as opposed to the left Lie algebra, the bracket in the right Lie algebra of $G$ will be denoted by $[\cdot, \cdot]^{R}$; there is a relation $[v, \mu]^{L}=-[v, \mu]^{R}$, and we recall once again that, for $z \in P_{\mid x}, \hat{z}: \mathfrak{g} \rightarrow \boldsymbol{g}_{\mid x}$ is an isomorphism of Lie algebras when $\mathfrak{g}$ is equipped with the right structure.

The partial connection in $P$ determines a partial connection $\lambda^{\prime}$ in the transitive Lie algebroid $A(P)$, and the system obtained $\left(A(P), A\left(P^{\prime}\right), \lambda^{\prime}\right)$ is a PFS-transitive Lie algebroid. In Theorem 11.3 the characteristic homomorphism $\Delta_{q^{\prime}} \#: H\left(\mathcal{W}(\boldsymbol{g}, \boldsymbol{h})_{q^{\prime}, I^{0}}\right) \rightarrow$ $H_{d R}(M)$ is obtained $\left(\boldsymbol{g}\right.$ and $\boldsymbol{h}$ being the Lie algebra bundles adjoint to $A(P)$ and $A\left(P^{\prime}\right)$, respectively). We compare $\hat{\Delta}_{1 q^{\prime}} \#$ with $\Delta_{q^{\prime}}$. For this purpose, consider the adjoint representation $\operatorname{Ad}_{P}: P \rightarrow L(\boldsymbol{g})[\mathrm{K} 2,5.3 .2]$ and the representation $\operatorname{Ad}_{P^{\prime}, \boldsymbol{g}}^{q^{\prime}}: P^{\prime} \rightarrow L\left(W(\boldsymbol{g} / \boldsymbol{h})_{q^{\prime}}\right)$, $\operatorname{Ad}_{P^{\prime}, \boldsymbol{g}}^{q^{\prime}}=\operatorname{Ad}_{P^{\prime}, \boldsymbol{g}}^{\wedge} \otimes \bigvee^{\leq q^{\prime}}\left(\operatorname{Ad}_{P} \mid P^{\prime}\right)^{\natural}$ (for $\operatorname{Ad}_{P^{\prime}, \boldsymbol{g}}^{\wedge}$ see $[\mathrm{K} 7$, Sec. 6]), induced by it. As in [K7, Sec. 6], we notice that the differential of $\operatorname{Ad}_{P^{\prime}, \boldsymbol{g}}^{q^{\prime}}$ is equal to the representation $\operatorname{ad}_{A\left(P^{\prime}\right), \boldsymbol{g}}^{q^{\prime}}=\operatorname{ad}_{A\left(P^{\prime}\right), \boldsymbol{g}}^{\wedge} \otimes \bigvee^{\leq q^{\prime}}\left(\operatorname{ad}_{A\left(P^{\prime}\right)} \mid A\left(P^{\prime}\right)\right)^{\natural}: A\left(P^{\prime}\right) \rightarrow A\left(W(\boldsymbol{g} / \boldsymbol{h})_{q^{\prime}}\right)$. Propositions 5.5.2-3 from $[\mathrm{K} 2]$ give a monomorphism

$$
\begin{aligned}
\bar{\kappa} & :\left(\bigwedge(\mathfrak{g} / \mathfrak{h})^{*} \otimes \bigvee^{\leq q^{\prime}} \mathfrak{g}^{*}\right)_{I_{H}} \cong\left(\operatorname{Sec} \bigwedge(\boldsymbol{g} / \boldsymbol{h})^{*} \otimes \bigvee^{\leq q^{\prime}} \boldsymbol{g}^{*}\right)_{I} \\
& \hookrightarrow\left(\operatorname{Sec} \bigwedge(\boldsymbol{g} / \boldsymbol{h})^{*} \otimes \bigvee^{\leq q^{\prime}} \boldsymbol{g}^{*}\right)_{I^{0}}
\end{aligned}
$$


defined by the formula $\bar{\kappa}(\psi)(x)=\operatorname{Ad}_{P^{\prime}, \boldsymbol{g}}^{q^{\prime}}(z)(x), z \in P_{\mid x}^{\prime}$, i.e.

$$
\bar{\kappa}(\psi)(x)=\left(\bigwedge[\hat{z}]^{*-1} \otimes \bigvee^{\leq q^{\prime}} \hat{z}^{*-1}\right)(\psi),
$$

which is an isomorphism when $P^{\prime}$ is connected.

THEOREM 14.1. $\bar{\kappa} \circ S$ (see (14.1)) commutes with the differentials $d_{q^{\prime}}$ and $d_{q^{\prime}, h}$, giving the commutative diagram

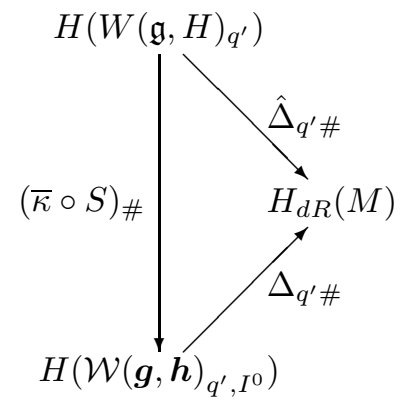

Proof. The evident commutativity of the diagram

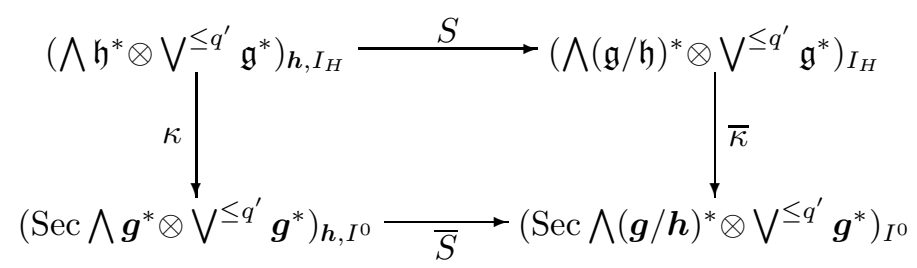

in which $\kappa(\psi)(x)=\left(\bigwedge \hat{z}^{*-1} \otimes \bigvee^{\leq q^{\prime}} \hat{z}^{*-1}\right)(\psi), z \in P_{\mid x}^{\prime}$, and of diagram (11.2), implies that the commutativity of $\bar{\kappa} \circ S$ with the differentials follows from the commutativity of $\kappa_{z}=\bigwedge \hat{z}^{*-1} \otimes \bigvee \hat{z}^{*-1}: W \mathfrak{g} \rightarrow W \boldsymbol{g}_{\mid x}$ with $d_{\mathfrak{g}}^{L}$ and $d_{x}$ which can be checked directly on generators:

$$
\begin{gathered}
\kappa_{z} \circ d_{\mathfrak{g}}^{L}\left(w^{*} \otimes 1\right)=1 \otimes \kappa_{z} w^{*}+\kappa_{z}\left(d_{\wedge} w^{*}\right) \otimes 1=d_{x} \circ \kappa_{z}\left(w^{*} \otimes 1\right) . \\
\iota_{v} \circ \kappa_{z} \circ d_{\mathfrak{g}}^{L}\left(1 \otimes w^{*}\right)=\kappa_{z}\left(1 \otimes \theta_{\hat{z}^{-1}(v)}^{L} w^{*}\right)=-\theta_{v} \circ \kappa_{z}\left(1 \otimes w^{*}\right)=\iota_{v} \circ d_{x} \circ \kappa_{z}\left(1 \otimes w^{*}\right) .
\end{gathered}
$$

Passing to the second part of our theorem we can write a diagram analogous to the one in the proof of $\left[K 7\right.$, Th. 6.1]. Analogously, we need the equality $j^{*}\left(\boldsymbol{k}_{q^{\prime}}\left(\omega_{P}\right)(\theta)\right)=\rho \circ$ $(d i)^{*}[\boldsymbol{k}]_{q^{\prime}}(\tilde{\kappa}(\theta))$ where $\tilde{\kappa}$ is the superposition $W(\mathfrak{g}, H)_{q^{\prime}} \rightarrow \mathcal{W}(\boldsymbol{g}, \boldsymbol{h})_{q^{\prime}, I^{0}} \rightarrow \mathcal{W}(\boldsymbol{g}) q^{\prime}, \boldsymbol{h}, I^{0}$, where $\omega_{P}$ is the connection form of an adapted connection. The equality (checked trivially on generators when one only knows the relations $\omega_{\mid x} \circ \pi_{\mid z}^{A}=\hat{z} \circ \omega_{P \mid z}, \Omega_{\mid z} \circ \bigwedge^{2} \pi_{\mid z}^{A}=\hat{z} \circ \Omega_{\mid z}$; $\omega$ is the connection form in $A(P)$ corresponding to $\omega_{P}$ ) is equivalent to the commutativity of the following simple diagram

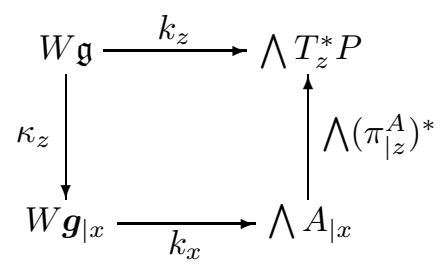


for any $x \in M$ and $z \in P_{\mid x}$. In this diagram, $k_{z}$ is a homomorphism of algebras fulfilling $k_{z}(\theta)=\left(k\left(\omega_{P}\right)(\theta)\right)_{z}$.

\section{PART III. APPENDIX: LOCAL PROPERTIES OF REGULAR LIE ALGEBROIDS, ELEMENTARY THEORY}

The fundamental role in the elementary proofs of some structural theorems on the local shape of regular Lie algebroids and their properties is played by a theorem concerning global solutions of some system of differential equations.

\section{Global smooth solution of some system of differential equations with} parameters. We prove (by methods of differential geometry) the existence of a global smooth solution of a system of linear partial differential equation with parameters, whose coefficients are smooth functions on the whole Euclidean space. The simple classical theorem asserts only the existence and uniqueness of some $C^{\infty}$ solution determined locally in some neighbourhood of an arbitrary point of the form $(0, y)$.

Denote the canonical coordinates on $\mathbb{R}^{m} \times \mathbb{R}^{n}$ by $\left(x^{1}, \ldots, x^{m}, y^{1}, \ldots, y^{n}\right)$. In the proof of the following theorem we use only the global existence of a solution of some system of ordinary differential equations without parameters and some elementary facts concerning the theory of foliations as for example:

(T) if $(M, F)$ is a foliated manifold and $N \hookrightarrow M$ is a transversal submanifold of $F$ such that each leaf $L$ of $F$ cuts $N$ in at most one point, then the sum of leaves passing through $N$ is also a submanifold of dimension $\operatorname{dim} F+\operatorname{dim} N$.

THEOREM 15.1. Let $C^{\infty}$ functions $b_{i}^{k}, a_{r i}^{k}: \mathbb{R}^{m} \times \mathbb{R}^{n} \rightarrow \mathbb{R}, r, k \leq q, i \leq m$, be given . Consider the following system of linear partial differential equations with parameters:

$$
\frac{\partial z^{k}}{\partial x^{i}}(x, y)=-b_{i}^{k}(x, y)+\sum_{r=1}^{q} a_{r i}^{k}(x, y) \cdot z^{r}, \quad k \leq q, i \leq m,
$$

satisfying the conditions of local integrability:

$$
\begin{gathered}
\frac{\partial b_{i}^{k}}{\partial x^{s}}-\frac{\partial b_{s}^{k}}{\partial x^{i}}=-\sum_{u=1}^{q} a_{u i}^{k} \cdot b_{s}^{u}+\sum_{u=1}^{q} a_{u s}^{k} \cdot b_{i}^{u}, \\
\frac{\partial a_{r i}^{k}}{\partial x^{s}}-\frac{\partial a_{r s}^{k}}{\partial x^{i}}=\sum_{u=1}^{q} a_{u s}^{k} \cdot a_{r i}^{u}-\sum_{u=1}^{q} a_{u i}^{k} \cdot a_{r s}^{u},
\end{gathered}
$$

$i, s \leq m, r \leq q$. Then, for every $C^{\infty}$ mapping $g: \mathbb{R}^{n} \rightarrow \mathbb{R}^{q}$, there exists exactly one globally defined $C^{\infty}$ solution $z: \mathbb{R}^{m} \times \mathbb{R}^{n} \rightarrow \mathbb{R}^{q}$ of $(15.1)$ such that $z(0, y)=g(y), y \in \mathbb{R}^{n}$.

Proof. Put $M=\mathbb{R}^{m} \times \mathbb{R}^{n} \times \mathbb{R}^{q}$ with coordinates $\left(x^{i}, y^{j}, z^{k}\right)$ and define $C^{\infty}$ differential 1-forms $\omega^{k}=d z^{k}+\sum_{i=1}^{m}\left(b_{i}^{k}(x, y)-\sum_{r=1}^{q} a_{r i}^{k}(x, y) \cdot z^{r}\right) d x^{i}$ on $M$. Consider the system $\left(\omega^{1}, \ldots, \omega^{q}, d y^{1}, \ldots, d y^{n}\right)$ of linearly independent differential 1 -forms on $M$. The distribution $E$ generated by this system of 1-forms has dimension $m$ and is integrable: $d\left(d y^{j}\right)=0, d \omega^{k}=\sum_{u} \alpha_{u}^{k} \wedge \omega^{u}+\sum_{j} \beta_{j}^{k} \wedge d y^{j}$, where

$$
\alpha_{u}^{k}=\sum_{i=1}^{m} a_{u i}^{k} \cdot d x^{i}, \quad \beta_{j}^{k}=\sum_{i=1}^{m}\left(-\frac{\partial b_{i}^{k}}{\partial y^{j}}+\sum_{r=1}^{q} \frac{\partial a_{r i}^{k}}{\partial y^{j}} \cdot z^{r}\right) \cdot d x^{i} .
$$


A $C^{\infty}$ mapping $z: \mathbb{R}^{m} \times \mathbb{R}^{n} \rightarrow \mathbb{R}^{q}$ is a solution of (15.1) if and only if, for each point $y_{0} \in \mathbb{R}^{n}$, the manifold $L_{y_{0}}(z):=\left\{\left(x, y_{0}, z\left(x, y_{0}\right)\right) ; x \in \mathbb{R}^{m}\right\}$ is an integral of $E$. We easily check that

$\left(^{*}\right)$ The space $E_{\mid(x, y, z)}$ lies on the plane $0 X Z$. Moreover, for $v=\sum_{i} a^{i} \cdot \frac{\partial}{\partial x^{i}}+\sum_{r} c^{r} \cdot \frac{\partial}{\partial z^{r}}$, we have $\omega^{k}(v)=c^{k}+\sum_{i}\left(b_{i}^{k}-\sum_{r} a_{r i}^{k} \cdot z^{r}\right) \cdot a^{i}$, which implies that, for $v \in E_{\mid(x, y, z)}$, if $a^{i}=0$ for all $i$, then $v=0$.

Let $L$ be the leaf of the distribution $E$, passing through a point $\left(x_{0}, y_{0}, z_{0}\right)$. Take the projection $\mathrm{pr}_{1}: L \rightarrow \mathbb{R}^{m}$. Since $\left(\mathrm{pr}_{1}\right)_{*}\left(\sum_{i} a^{i} \cdot \frac{\partial}{\partial x^{i}}+\sum_{r} c^{r} \cdot \frac{\partial}{\partial z^{r}}\right)=\sum_{i} a^{i} \cdot \frac{\partial}{\partial x^{i}},\left(^{*}\right)$ above gives that $\left(\mathrm{pr}_{1}\right)_{*}$ is an isomorphism at each point, therefore $\mathrm{pr}_{1}$ is a local diffeomorphism. Since $\mathbb{R}^{m}$ is simply connected, to see that $\mathrm{pr}_{1}$ is a diffeomorphism, we only have to notice that $\mathrm{pr}_{1}$ is a covering, but this is equivalent to the fulfilment of the following two properties (see lemma 15.2 below):

- the surjectivity of this projection, and

— the lifting property for smooth paths.

To check the surjectivity, take $x_{1} \in \mathbb{R}^{m}$ and $\lambda:=x_{1}-x_{0}$. Define the embedding $\varphi: \mathbb{R} \times \mathbb{R}^{q} \rightarrow \mathbb{R}^{m} \times \mathbb{R}^{n} \times \mathbb{R}^{q}, \varphi(t, z)=\left(x_{0}+t \cdot \lambda, y_{0}, z\right)$, and calculate: $\varphi^{*}\left(d x^{i}\right)=$ $\lambda^{i} \cdot d t, \varphi^{*} \omega^{k}=d z^{k}+\sum_{i}\left(\tilde{b}_{i}^{k}-\sum_{r=1}^{q} \tilde{a}_{r i}^{k} \cdot z^{r}\right) \cdot \lambda^{i} \cdot d t, k \leq q$, where $\tilde{b}_{i}^{k}, \tilde{a}_{r i}^{k}: \mathbb{R} \rightarrow \mathbb{R}$ are defined by $\tilde{b}_{i}^{k}(t)=b_{i}^{k}\left(x_{0}+t \cdot \lambda, y_{0}\right), \tilde{a}_{r i}^{k}(t)=a_{r i}^{k}\left(x_{0}+t \cdot \lambda, y_{0}\right)$. The 1 -forms $\varphi^{*} \omega^{k}$ correspond to the linear nonhomogeneous system of ordinary differential equations of the first order

$$
\frac{d z^{k}(t)}{d t}=-\sum_{i} \tilde{b}_{i}^{k}(t) \cdot \lambda^{i}+\sum_{r}\left(\sum_{i} \tilde{a}_{r i}^{k}(t) \cdot \lambda^{i}\right) \cdot z^{r}(t), \quad k \leq q .
$$

Consider the initial condition $z^{k}(0)=z_{0}^{k}$. The well-known classical theorem states that there exists a unique globally determined (on the whole space $\mathbb{R}$ ) solution $z=\left(z^{1}, \ldots, z^{q}\right.$ ) of this system, satisfying the initial condition. As previously,

$$
\tilde{L}=\{(t, z(t)) ; t \in \mathbb{R}\}
$$

is a maximal integral of the one-dimensional distribution determined by the system of 1forms $\left(\varphi^{*} \omega^{1}, \ldots, \varphi^{*} \omega^{q}\right)$. The mapping $\kappa: \mathbb{R} \rightarrow \tilde{L}, t \mapsto(t, z(t))$, is a global trivialization of $\tilde{L}$. Using $\left(^{*}\right)$ above, we can easily prove that $\varphi[\tilde{L}]$ is an integral manifold of the distribution $E$. Then $x_{1}=\operatorname{pr}_{1}\left(x_{1}, y_{0}, z(1)\right)=\operatorname{pr}_{1} \circ \varphi \circ \kappa(1) \in \operatorname{pr}_{1}[\tilde{L}]$. To check the lifting property for smooth paths, take a smooth path $\tau=\left(\tau^{1}, \ldots, \tau^{m}\right): \mathbb{R} \rightarrow \mathbb{R}^{m}$, such that $\tau(0)=x_{0}$. The lifting of $\tau$ to the point $\left(x_{0}, y_{0}, z_{0}\right)$ is a smooth path $\tau^{*}: \mathbb{R} \rightarrow \mathbb{R}^{m} \times \mathbb{R}^{n} \times \mathbb{R}^{q}$ such that $\tau^{*}(0)=\left(x_{0}, y_{0}, z_{0}\right), \frac{d \tau^{*}(t)}{d t} \in E_{\mid \tau^{*}(t)}$ and $\operatorname{pr}_{1} \circ \tau^{*}=\tau$ (i.e. $\tau^{* i}=\tau^{i}, i \leq m$ ). Since $\frac{d \tau^{*}(t)}{d t} \in E_{\mid \tau^{*}(t)}$ if and only if $\omega^{k}\left(\frac{d \tau^{*}(t)}{d t}\right)=0$ and $d y^{j}\left(\frac{d \tau^{*}(t)}{d t}\right)=0$, we see that $\tau^{*}$ is the lifting of $\tau$ if and only if $\tau^{* i}=\tau^{i}, i \leq m, \tau^{* m+j} \equiv y_{o}^{j}, j \leq n$, and

$$
\frac{d \tau^{* m+n+k}(t)}{d t}=-\sum_{i=1}^{m} b_{i}^{k}\left(\tau(t), y_{0}\right) \cdot \frac{d \tau^{i}(t)}{d t}+\sum_{r=1}^{q} \sum_{i=1}^{m} a_{r i}^{k}\left(\tau(t), y_{0}\right) \cdot \frac{d \tau^{i}(t)}{d t} \cdot \tau^{* m+n+r}(t),
$$

$k \leq q$, with the initial condition $\tau^{* k}(0)=z_{0}^{k}$. According to the above-mentioned classical theorem, there exists exactly one globally determined (on the whole space $\mathbb{R}$ ) solution 
$\left(\tau^{* m+n+1}, \ldots, \tau^{* m+n+q}\right)$ of this system, satisfying the initial condition. The path

$$
\tau^{*}(t)=\left(\tau^{1}(t), \ldots, \tau^{m}(t), y_{0}^{1}, \ldots, y_{0}^{n}, \tau^{* m+n+1}(t), \ldots, \tau^{* m+n+q}(t)\right),
$$

$t \in \mathbb{R}$, is the desired lifting.

Take $g: \mathbb{R}^{n} \rightarrow \mathbb{R}^{q}$ and a submanifold $N=\left\{(0, y, g(y)) ; y \in \mathbb{R}^{n}\right\} \subset M$. Using (*) as above we assert that $N$ is a transversal of the foliation $E, T_{(0, y, g(y))} N \cap E_{\mid(0, y, g(y))}=0$. Denote by $L_{y}$ the leaf of $E$ passing through $(0, y, g(y))$, and define $\bar{L}=\bigcup_{y \in \mathbb{R}^{n}} L_{y}$. $L$ is, of course, a submanifold of $M$ (see (T) above). We prove that $\operatorname{pr}=\operatorname{pr}_{1,2} \mid \bar{L}: \bar{L} \rightarrow$ $\mathbb{R}^{m} \times \mathbb{R}^{n}$ is a diffeomorphism. Clearly, pr is a smooth bijection (see the previous step). Take $\left(x_{0}, y_{0}, z_{0}\right) \in \bar{L}$ and $v \in T_{\left(x_{0}, y_{0}, z_{0}\right)} \bar{L}$ such that $\operatorname{pr}_{*}(v)=0$. The equality $v=0$ is what we need to show; $v$ is of the form $v=\sum_{k} c^{k} \cdot \frac{\partial}{\partial z^{k}}$. Consider two complete transversals $T_{0}$ and $T_{x_{0}}$ of $E$ determined by the equations $x=0$ and $x=x_{0}$, respectively, and a diffeomorphism $\varphi: T_{x_{0}} \rightarrow T_{0}$ such that the points $\left(x_{0}, y, z\right)$ and $\varphi\left(x_{0}, y, z\right)$ lie on one of the leaves of $E$. The diffeomorphism $\varphi$ is, of course, uniquely determined. The vector $v$ is tangent to $T_{x_{0}}$. Since $\varphi$ is of the form $\varphi\left(x_{0}, y, z\right)=(0, y, \tilde{\varphi}(y, z))$ for some function $\tilde{\varphi}$, therefore $w:=\varphi_{*}(v)$ is of the form $w=\sum_{k} \tilde{c}^{k} \cdot \frac{\partial}{\partial z^{k}}$, i.e. its coordinates with respect to the vectors $\frac{\partial}{\partial y^{j}}$ are zero. On the other hand, $v \in T_{\left(x_{0}, y_{0}, z_{0}\right)} \bar{L} \cap T_{\left(x_{0}, y_{0}, z_{0}\right)} T_{x_{0}}=$ $T_{\left(x_{0}, y_{0}, z_{0}\right)}\left(\bar{L} \cap T_{x_{0}}\right)\left(\bar{L} \cap T_{x_{0}}\right.$ is equal to $\varphi^{-1}[N]$ and is a submanifold) and $\varphi\left(x_{0}, y_{0}, z_{0}\right)=$ $\left(0, y_{0}, g\left(y_{0}\right)\right)$; then $w \in T_{\left(0, y_{0}, g\left(y_{0}\right)\right)} N$. However, $N$ is the image of the mapping $\psi: \mathbb{R}^{n} \rightarrow$ $\mathbb{R}^{m} \times \mathbb{R}^{n} \times \mathbb{R}^{q}, y \mapsto(0, y, g(y))$, so, $w=\psi_{* y_{0}}(\bar{w})$ for some $\bar{w} \in T_{y_{0}} \mathbb{R}^{n}$. Therefore $0=$ $\operatorname{pr}_{*}(w)=\operatorname{pr}_{*}\left(\psi_{* y_{0}}(\bar{w})\right)=\bar{w}$, which implies $w=0$ and, next, $v=0$.

Let $\operatorname{pr}_{3}: \mathbb{R}^{m} \times \mathbb{R}^{n} \times \mathbb{R}^{q} \rightarrow \mathbb{R}^{q}$ denote the projection onto the last factor. The mapping $z: \mathbb{R}^{m} \times \mathbb{R}^{n} \rightarrow \mathbb{R}^{q}$ equal to $z:=\operatorname{pr}_{3} \circ(\mathrm{pr})^{-1}$ is the required solution of (15.1).

LEMma 15.2. A surjective local diffeomorphism $\pi: M \rightarrow N$ of connected manifolds is a covering if and only if it possesses the lifting property for smooth paths.

Proof. This clearly follows from Ehresmann's lemma in P. Molino's version [Mo2, p. 114]. We only need to take an arbitrary family $L_{c}^{\prime}$ of smooth compact support vector fields on $N$ generating at each point $x \in N$ the entire tangent space $T_{x} N$. Under the lifting property for smooth paths, the family $L_{c}$ of the liftings of $X \in L_{c}^{\prime}$ consists of complete fields.

16. Trivial regular Lie algebroids. If the anchor is equal to zero, a Lie algebroid is called [Ma1] completely intransitive. It is simply a bundle of Lie algebras (Lie algebras $A_{\mid x}$ and $A_{\mid y}, x, y \in M$, need not be isomorphic, although the bracket $[\xi, \eta]$ of $C^{\infty}$ crosssections of $A$, defined pointwise: $[\xi, \eta]_{x}=\left[\xi_{x}, \eta_{x}\right]$, is $C^{\infty}$, too).

One of the most important constructions is the inverse-image $f^{\wedge} A$ by a homomorphism of foliated manifolds $f:\left(M^{\prime}, E^{\prime}\right) \rightarrow(M, E)[\mathrm{K} 2]: f^{\wedge} A=E^{\prime} \times_{\left(f_{*}, \gamma\right)} A=$ $\left\{(v, w) \in E^{\prime} \times A ; f_{*}(v)=\gamma(w)\right\} \subset E^{\prime} \oplus f^{*} A$,

$$
\begin{aligned}
& \llbracket\left(X, \sum_{j} f^{j} \cdot \xi_{j} \circ f\right),\left(Y, \sum_{k} g^{k} \cdot \eta_{k} \circ f\right) \rrbracket \\
& =\left([X, Y], \sum_{j, k} f^{j} \cdot g^{k} \cdot \llbracket \xi_{j}, \eta_{k} \rrbracket \circ f+\sum_{k} X\left(g^{k}\right) \cdot \eta_{k} \circ f-\sum_{j} Y\left(f^{j}\right) \cdot \xi_{j} \circ f\right)
\end{aligned}
$$


for $f^{j}, g^{k} \in \Omega^{0}\left(M^{\prime}\right), \xi_{j}, \eta_{k} \in \operatorname{Sec} A$. The projection onto the first component $\operatorname{pr}_{1}: f^{\wedge} A=$ $E^{\prime} \times_{\left(f_{*}, \gamma\right)} A \rightarrow E^{\prime}$ serves as the anchor. A nonstrong homomorphism $H: A^{\prime} \rightarrow A$ of regular Lie algebroids (over $f:\left(M^{\prime}, E^{\prime}\right) \rightarrow(M, E)$ ) [K2] can be defined as a superposition $A^{\prime} \stackrel{\bar{H}}{\rightarrow} f^{\wedge} A \stackrel{\kappa}{\rightarrow} A$ of some strong homomorphism $\bar{H}$ and the canonical one $\kappa=\mathrm{pr}_{2}$. Here are the basic properties of the inverse-image operation:

$-(g \circ f)^{\wedge} A \cong f^{\wedge}\left(g^{\wedge} A\right)$

— if $i_{x}:\{x\} \hookrightarrow M$ is the inclusion, then $i_{x}^{\wedge} A \cong \boldsymbol{g}_{\mid x}$.

Definition 16.1. By a trivial regular lie algebroid over $(M, E)$ we shall mean each algebroid isomorphic to $f^{\wedge} A$ for some completely intransitive Lie algebroid $A$.

Each $C^{\infty}$ constant dimensional and completely integrable distribution $E$ on a manifold $M$ is a regular Lie algebroid being, of course, trivial.

EXAMPLE 16.2 (Transitive trivial Lie algebroid). Let a trivial Lie algebroid $f^{\wedge} A$ (where $A$ is a completely intransitive Lie algebroid on a manifold $N$ ) be transitive (this means that it is over $(M, T M))$. Then $f$ is a constant mapping, say $f(x) \equiv y$. Put $\bar{y}: M \rightarrow\{y\}, x \mapsto y$, and let $i_{y}:\{y\} \hookrightarrow N$ be the inclusion. Then

$$
f^{\wedge} A \cong \bar{y}^{\wedge}\left(i_{y}^{\wedge} A\right) \cong \bar{y}^{\wedge}(\mathfrak{g})=T M \times \mathfrak{g} \quad\left(\mathfrak{g}=\boldsymbol{g}_{\mid y}\right) .
$$

Clearly, $\bar{y}^{\wedge}(\mathfrak{g})$ is the usual trivial transitive Lie algebroid [NVQ], [K2].

Example 16.3. Consider two manifolds $M$ and $N$, the projection $\operatorname{pr}_{2}: M \times N \rightarrow N$ and a vector bundle of Lie algebras $\mathfrak{f}$ on $N$ considered as a completely intransitive Lie algebroid. Of course, $\mathrm{pr}_{2}:(M \times N, T M \times 0) \rightarrow(N, 0)$ is a homomorphism of foliated manifolds. We see that the inverse-image $\operatorname{pr}_{2}^{\wedge}(\mathfrak{f})$ is equal to $(T M \times 0) \oplus \operatorname{pr}_{2}^{*}(\mathfrak{f})$. Each crosssection of $\operatorname{pr}_{2}^{\wedge}(\mathfrak{f})$ is a sum of cross-sections of the form $\left(X, f \cdot \sigma \circ \mathrm{pr}_{2}\right)$ for $X \in \operatorname{Sec}(T M \times 0)$, $f \in \Omega^{0}(M \times N), \sigma \in \operatorname{Sec} \mathfrak{f}$. Therefore the structure of a Lie algebra in $\operatorname{Sec}_{\operatorname{pr}}^{\wedge}(\mathfrak{f})$ is determined uniquely by demanding that

$$
\begin{aligned}
& \llbracket\left(X, f \cdot \sigma \circ \mathrm{pr}_{2}\right),\left(Y, g \cdot \eta \circ \mathrm{pr}_{2}\right) \rrbracket \\
& =\left([X, Y], f \cdot g \cdot \llbracket \xi, \eta \rrbracket \circ \mathrm{pr}_{2}+X(g) \cdot \eta \circ \mathrm{pr}_{2}-Y(f) \cdot \xi \circ \mathrm{pr}_{2}\right) .
\end{aligned}
$$

17. A regular Lie algebroid over $\left(\mathbb{R}^{p} \times \mathbb{R}^{q}, T \mathbb{R}^{p} \times 0\right)$ possesses a flat connection. The following theorem generalizes the result of K. Mackenzie [Ma1] concerning transitive Lie algebroids (see also $[\mathrm{A}-\mathrm{M}]$ ).

THEOREM 17.1. Every regular Lie algebroid over $\left(\mathbb{R}^{p} \times \mathbb{R}^{q}, T \mathbb{R}^{p} \times 0\right)$ possesses a flat connection.

Proof. Consider any regular Lie algebroid $B$ over $\left(\mathbb{R}^{p} \times \mathbb{R}^{q}, T \mathbb{R}^{p} \times 0\right)$, let $\boldsymbol{g}=\operatorname{ker} \gamma$ where $\gamma: B \rightarrow T \mathbb{R}^{p} \times 0$ is the anchor. Denote by $\left(y^{1}, \ldots, y^{p}, y^{p+1}, \ldots, y^{p+q}\right)$ the canonical coordinates on $\mathbb{R}^{p} \times \mathbb{R}^{q}$. We prove, by induction with respect to $n=1,2, \ldots, p$, that there exist linearly independent cross-sections $Y_{1}, \ldots, Y_{n}$ of $B$ such that

$$
\gamma \circ Y_{i}=\frac{\partial}{\partial y^{i}}, \quad \llbracket Y_{i}, Y_{j} \rrbracket=0, \quad i, j \leq n .
$$

Of course, the cross-sections $Y_{1}, \ldots, Y_{n}$ fulfilling (17.1) for $n=p$ give rise to a connection $\lambda: T \mathbb{R}^{p} \times 0 \rightarrow B$ defined uniquely by demanding that $\lambda \circ \frac{\partial}{\partial y^{i}}=Y_{i}, i \leq p$. Clearly, $\lambda$ is 
flat. (17.1) is evidently valid for $n=1$. Let (17.1) be valid for some $m \in\{1, \ldots, p-1\}$. We prove that it is true for $m+1$. For this purpose, take linearly independent cross-sections $X_{1}, \ldots, X_{q^{\prime}}, Y_{1}, \ldots, Y_{m}$ of $B$ such that $X_{1}, \ldots, X_{q^{\prime}}$ form a basis of $\boldsymbol{g}$ and $Y_{1}, \ldots, Y_{m}$ fulfil (17.1) for $n=m$. Let $\bar{Y}$ be an arbitrary cross-section of $B$ for which $\gamma \circ \bar{Y}=\frac{\partial}{\partial y^{m+1}}$. We shall find $C^{\infty}$ functions $z^{1}, \ldots, z^{q^{\prime}} \in \Omega^{0}\left(\mathbb{R}^{p} \times \mathbb{R}^{q}\right)$ such that $\llbracket Y_{i}, Y_{m+1} \rrbracket=0, i \leq m$, where $Y_{m+1}:=\sum_{i=1}^{q^{\prime}} z^{i} \cdot X_{i}+\bar{Y}$. To this end, put $\llbracket Y_{i}, \bar{Y} \rrbracket=\sum_{k=1}^{q^{\prime}} b_{i}^{k} \cdot X_{k}, i \leq m$, and $\left[X_{i}, X_{j}\right]=\sum_{k=1}^{q^{\prime}} a_{i j}^{k} \cdot X_{k}, i, j \leq q^{\prime}$. Then the equations $\llbracket Y_{i}, Y_{m+1} \rrbracket=0, i \leq m$, are all equivalent to the following system of differential equations with parameters $y^{m+1}, \ldots, y^{p+q}$ :

$$
\begin{aligned}
& \frac{\partial z^{k}}{\partial y^{i}}\left(\ldots, y^{i}, \ldots, y^{m+1}, \ldots, y^{p+q}\right) \\
& =-b_{i}^{k}\left(\ldots, y^{i}, \ldots, y^{m+1}, \ldots, y^{p+q}\right)+\sum_{r=1}^{q^{\prime}} a_{r i}^{k}\left(\ldots, y^{i}, \ldots, y^{m+1}, \ldots, y^{p+q}\right) \cdot z^{r},
\end{aligned}
$$

$k \leq q^{\prime}$ and $i \leq m$. A system like this is always uniquely integrable. The following conditions of local integrability hold by the Jacobi identities: $\llbracket \llbracket Y_{s}, \bar{Y} \rrbracket, Y_{i} \rrbracket+c y c l=0$ and $\llbracket \llbracket X_{r}, Y_{s} \rrbracket, Y_{i} \rrbracket+c y c l=0$. According to Theorem 15.1, the system has a global solution $\left(z^{1}, \ldots, z^{q^{\prime}}\right) \in \Omega^{0}\left(\mathbb{R}^{p} \times \mathbb{R}^{q}, \mathbb{R}^{q^{\prime}}\right)$ fulfilling an arbitrary initial condition. To prove our lemma, take the system $\left(Y_{1}, \ldots, Y_{m}, Y_{m+1}\right)$ where $Y_{m+1}=\sum_{i=1}^{q^{\prime}} z^{i} \cdot X_{i}+\bar{Y}$.

18. A regular Lie algebroid over $\left(\mathbb{R}^{p} \times \mathbb{R}^{q}, T \mathbb{R}^{p} \times 0\right)$ is trivial. Let $B$ be any regular Lie algebroid over $\left(\mathbb{R}^{p} \times \mathbb{R}^{q}, T \mathbb{R}^{p} \times 0\right)$. We begin with the following lemma.

LEMma 18.1 (cf. $[\mathrm{A}-\mathrm{M}])$. Let $Y_{1}, \ldots, Y_{p}$ be cross-sections of $B$ satisfying conditions (17.1) for $n=p$. Then there exists a basis $\left(X_{1}, \ldots, X_{q^{\prime}}\right)$ of $\boldsymbol{g}$ such that

$$
\llbracket Y_{i}, X_{j} \rrbracket=0, \quad i \leq p, j \leq q^{\prime} .
$$

Proof. The vector bundle $\boldsymbol{g}$ being over $\mathbb{R}^{p} \times \mathbb{R}^{q}$ is trivial, therefore it possesses a global basis $\left(\bar{X}_{1}, \ldots, \bar{X}_{q^{\prime}}\right)$ of cross-sections. We find $C^{\infty}$ functions $f_{j}^{r}, j, r \leq q^{\prime}$, such that

$\left(1^{0}\right) \operatorname{det}\left[f_{j}^{r}(x)\right] \neq 0$ for all $x \in \mathbb{R}^{p} \times \mathbb{R}^{q}$,

$\left(2^{0}\right)$ the cross-sections $X_{j}=\sum_{r} f_{j}^{r} \cdot \bar{X}_{r}$ satisfy (18.1) above.

Condition $\left(2^{0}\right)$ is equivalent to $\frac{\partial}{\partial y^{i}}\left(f_{j}^{k}\right)+\sum_{r} f_{j}^{r} \cdot a_{r i}^{k}=0, i \leq p, k, j \leq q^{\prime}$, where $a_{r i}^{k}$ are defined by $\llbracket Y_{i}, \bar{X}_{r} \rrbracket=\sum_{k} a_{r i}^{k} \cdot \bar{X}_{k}$. Consider the following system of partial differential equations (with parameters $\left(y^{p+1}, \ldots, y^{p+q}\right)$ )

$$
\frac{\partial z^{k}}{\partial y^{i}}\left(\ldots, y^{i}, \ldots, y^{p+1}, \ldots, y^{p+q}\right)=-\sum_{r=1}^{q^{\prime}} a_{r i}^{k}\left(\ldots, y^{i}, \ldots, y^{p+1}, \ldots, y^{p+q}\right) \cdot z^{r}
$$

$k \leq q^{\prime}, i \leq p$. The conditions of its local integrability are equivalent to the true equality $\llbracket \bar{X}_{r}, \llbracket Y_{i}, Y_{s} \rrbracket \rrbracket=0$. Consider $q^{\prime}$ initial conditions of the form

$$
z^{k}(0, y)=\delta_{j}^{k}, \quad k=1, \ldots, q^{\prime}, y \in \mathbb{R}^{q},
$$

indexed by $j=1, \ldots, q^{\prime}$. Let $f_{j}^{1}, \ldots, f_{j}^{q^{\prime}}$ be the solution of $(18.2)$ defined on $\mathbb{R}^{p} \times \mathbb{R}^{q}$ and satisfying condition (18.3) (the existence is obtained by Th. 15.1). It remains to show condition $\left(1^{0}\right)$ above. Assume to the contrary that, at some point $\left(x_{0}, y_{0}\right) \in \mathbb{R}^{p} \times \mathbb{R}^{q}$, 
$\operatorname{det}\left[f_{j}^{r}\left(x_{0}, y_{0}\right)\right]=0$. This means that the vectors $\left[f_{j}^{1}\left(x_{0}, y_{0}\right), \ldots, f_{j}^{q^{\prime}}\left(x_{0}, y_{0}\right)\right], j \leq q^{\prime}$, are linearly dependent. Changing, if necessary, the numbering of the initial conditions, we may assume that $\left[f_{1}^{1}\left(x_{0}, y_{0}\right), \ldots, f_{1}^{q^{\prime}}\left(x_{0}, y_{0}\right)\right]=\sum_{j=2}^{q^{\prime}} C^{j} \cdot\left[f_{j}^{1}\left(x_{0}, y_{0}\right), \ldots, f_{j}^{q^{\prime}}\left(x_{0}, y_{0}\right)\right]$. Fix in equations $(18.2)$ the parameters $\left(y^{p+1}, \ldots, y^{p+q}\right)$ by setting them equal to $y_{0}$. In the equations thus obtained (without parameters) consider the initial condition $z^{k}\left(x_{0}\right)=$ $\sum_{j=2}^{q^{\prime}} C^{j} \cdot f_{j}^{k}\left(x_{0}, y_{0}\right), k \leq q^{\prime}$. It is clearly fulfilled by the solution $\left(f_{1}^{1}\left(\cdot, y_{0}\right), \ldots, f_{1}^{q^{\prime}}\left(\cdot, y_{0}\right)\right)$ and, simultaneously, by the family $g^{k}=\sum_{j=2}^{q^{\prime}} C^{j} \cdot f_{j}^{k}\left(\cdot, y_{0}\right), k \leq q^{\prime}$, which is also a solution of the system of differential equations obtained. By the uniqueness of solutions of this system, $f_{1}^{k}\left(\cdot, y_{0}\right)=g^{k}$ for $k \leq q^{\prime}$. In particular, we have $f_{1}^{k}\left(0, y_{0}\right)=g^{k}(0)$, which means that the vector $\left[f_{1}^{1}\left(0, y_{0}\right), \ldots, f_{1}^{q^{\prime}}\left(0, y_{0}\right)\right]$ is a linear combination of $\left[f_{j}^{1}\left(0, y_{0}\right), \ldots, f_{j}^{q^{\prime}}\left(0, y_{0}\right)\right]$, $2 \leq j \leq q^{\prime}$, which is not possible.

ThEOREM 18.2. Every regular Lie algebroid $B$ over $\left(\mathbb{R}^{p} \times \mathbb{R}^{q}, T \mathbb{R}^{p} \times 0\right)$ is trivial.

Proof. Assume that the cross-sections $\left(X_{1}, \ldots, X_{q^{\prime}}, Y_{1}, \ldots, Y_{p}\right)$ satisfy conditions (17.1) and (18.1). Then the structure functions $c_{i j}^{k}$ such that $\llbracket X_{i}, X_{j} \rrbracket=\sum_{k} c_{i j}^{k} \cdot X_{k}$ are constant on plaques of the foliation $T \mathbb{R}^{p} \times 0$, i.e. on the submanifolds $\mathbb{R}^{p} \times\{*\}$. Indeed, $0=\llbracket Y_{s}, \llbracket X_{i}, X_{j} \rrbracket \rrbracket+\operatorname{cycl}=\sum_{k} \frac{\partial}{\partial y^{s}}\left(c_{i j}^{k}\right) \cdot X_{k}$. The mapping $\lambda: T \mathbb{R}^{p} \times 0 \rightarrow B$ given by $\lambda \circ \frac{\partial}{\partial y^{i}}=Y_{i}$ is a flat connection. Take the embedding $\iota: \mathbb{R}^{q} \rightarrow \mathbb{R}^{p} \times \mathbb{R}^{q}, y \mapsto(0, y)$, and put $\mathfrak{f}=\iota^{*} \boldsymbol{g}$. The system $\left(\bar{X}_{1}, \ldots, \bar{X}_{q^{\prime}}\right)$ of cross-sections given by $\bar{X}_{i}(y)=X_{i}(0, y)$ serves as a basis of $\mathfrak{f}$. Consider the projection $\mathrm{pr}_{2}: \mathbb{R}^{p} \times \mathbb{R}^{q} \rightarrow \mathbb{R}^{q}$ and an isomorphism of vector bundles $\varphi: \operatorname{pr}_{2}^{*}(\mathfrak{f}) \rightarrow \boldsymbol{g}$ such that $\varphi_{\mid(x, y)}\left(\sum_{i} a^{i} \cdot \bar{X}_{i}(y)\right)=\sum_{i} a^{i} \cdot X_{i}(x, y)$. Next, we shall treat $\mathfrak{f}$ as a completely nontransitive Lie algebroid over $\mathbb{R}^{q}$. Using Ex. 16.3 we can easily prove that the isomorphism of vector bundles

$$
F: \operatorname{pr}_{2}^{\wedge}(\mathfrak{f})=\left(T \mathbb{R}^{p} \times 0\right) \oplus \operatorname{pr}_{2}^{*}(\mathfrak{f}) \rightarrow B, \quad(v, w) \mapsto \lambda(v)+\varphi(w),
$$

is an isomorphism of regular Lie algebroids.

As a simple corollary we obtain the well known fact

Corollary 18.3. Any transitive Lie algebroid over $\mathbb{R}^{n}$ is isomorphic to the trivial Lie algebroid $T \mathbb{R}^{n} \times \mathfrak{g}$ for some Lie algebra $\mathfrak{g}$.

Using a distinguished chart $U \stackrel{\varphi}{\rightarrow} \mathbb{R}^{p} \times \mathbb{R}^{q}$ of a given regular foliation $E$ on a manifold $M$ with $\varphi^{-1}\left[\mathbb{R}^{p} \times\{*\}\right]$ as plaques we obtain as a corollary that for any regular Lie algebroid $A$ on $M$ and any point $x \in M$ there exists a neighbourhood $U$ of $x$ such that $A$ restricted to $U$ (i.e. $i^{\wedge} A$ where $i: U \hookrightarrow M$ is the inclusion) is trivial.

\section{Representations of the trivial transitive Lie algebroid on $\mathbb{R}^{n}$ on a vector} bundle. The main results of this section easily follow from a more general Theorem IV.1.19 by K. Mackenzie [Ma1]. We give here an elementary proof using Th. 15.1.

With a real vector bundle $\mathfrak{f}$ over $M$ there is associated a transitive Lie algebroid $A(\mathfrak{f})$ over $M$ [Ma1], [K2], whose fibre over $x \in M$ consists of all f-vectors at $x$, i.e. linear homomorphisms $l: \operatorname{Sec} \mathfrak{f} \rightarrow \mathfrak{f}_{\mid x}$ for which there exists a vector $u \in T_{x} M$ such that $l(f \cdot \nu)=f(x) \cdot l(\nu)+u(f) \cdot \nu(x), f \in \Omega^{0}(M)$ and $\nu \in$ Secf . The vector $u$ is determined by $l$ uniquely and serves as its anchor. A local trivialization of $A(\mathfrak{f})$ gives the mapping $\bar{\psi}: T U \times \operatorname{End}(V) \rightarrow A(\mathfrak{f})_{\mid U}(V$ is the typical fibre of $\mathfrak{f})$ defined for a given local 
trivialization $\psi: U \times V \rightarrow \mathfrak{f}_{\mid U}$ of $\mathfrak{f}$ by the formula $\bar{\psi}(v, a)(\nu)=\psi_{\mid x}\left(v\left(\nu_{\psi}\right)+a\left(\nu_{\psi}(x)\right)\right)$ where, for $\nu \in \operatorname{Sec} \mathfrak{f}, \nu_{\psi}: U \rightarrow V$ is a function $x \mapsto \psi_{\mid x}^{-1}\left(\nu_{x}\right)$ [K2, Lemma 5.4.4]. A crosssection $\xi \in \operatorname{Sec} A(\mathfrak{f})$ determines a covariant differential operator $\mathcal{L}_{\xi}: \operatorname{Sec}(\mathfrak{f}) \rightarrow \operatorname{Sec}(\mathfrak{f})$ by the formula $\mathcal{L}_{\xi}(\nu)(x)=\xi_{x}(\nu)$. The correspondence $\xi \mapsto \mathcal{L}_{\xi}$ is $1-1$. The bracket $\llbracket \cdot, \cdot \rrbracket$ is defined classically (from the point of view of differential operators). The Lie algebra bundle adjoint of $A(\mathfrak{f})$ can be identified with the vector bundle End(f). Lemma 5.4.4 from [K2] mentioned above asserts also that $\bar{\psi}$ is an isomorphism of Lie algebroids. In particular, taking $\psi=\operatorname{id}_{\mathbb{R}^{n} \times V}$, we find that the Lie algebroid $A\left(\mathbb{R}^{n} \times V\right)$ of the trivial vector bundle $\mathfrak{f}=\mathbb{R}^{n} \times V$ is isomorphic to the trivial algebroid $T \mathbb{R}^{n} \times \operatorname{End}(V)$ via the canonical isomorphism $\mathcal{L}: T \mathbb{R}^{n} \times \operatorname{End}(V) \rightarrow A\left(\mathbb{R}^{n} \times V\right)$ defined by the formula: $\mathcal{L}_{\mid x}(v, a)(\nu)=v(\nu)+a\left(\nu_{x}\right)$. Denote by $\mathcal{L}_{(X, \sigma)}$ the differential operator determined by the cross-section $\mathcal{L} \circ(X, \sigma)$ of $A\left(\mathbb{R}^{n} \times V\right)$, where $X \in \mathfrak{X}\left(\mathbb{R}^{n}\right)$ and $\sigma \in \Omega^{0}\left(\mathbb{R}^{n}, \operatorname{End}(V)\right)$. Clearly

$$
\mathcal{L}_{(X, \sigma)}: \Omega^{0}\left(\mathbb{R}^{n} \times V\right) \rightarrow \Omega^{0}\left(\mathbb{R}^{n} \times V\right), \quad \nu \mapsto X(\nu)+\sigma(\nu) .
$$

By a representation of a Lie algebroid $A$ on $\mathfrak{f}$ (both over $M$ ) we mean a strong homomorphism $T: A \rightarrow A(\mathfrak{f})$ of Lie algebroids [Ma1]. $T$ induces a linear homomorphism $T^{+}: \boldsymbol{g} \rightarrow \operatorname{End}(\mathfrak{f})$ of vector bundles of Lie algebras [K2]. A cross-section $\nu \in \operatorname{Sec}(\mathfrak{f})$ is called $T$-invariant if $T(v)(\nu)=0$ for all $v \in A$. The space of all $T$-invariant cross-sections is denoted by $(\operatorname{Sec}(\mathfrak{f}))_{I^{0}(T)}$ (or, briefly, by $\left.(\operatorname{Sec}(\mathfrak{f}))_{I^{0}}\right)$.

THEOREM 19.1 (cf. [Ma1]). Let $\bar{T}: T \mathbb{R}^{n} \times \mathfrak{g} \rightarrow A(\mathfrak{f})$ be any representation of the trivial Lie algebroid $T \mathbb{R}^{n} \times \mathfrak{g}$ on $\mathfrak{f}$. Then, for each $\bar{T}_{\mid x}^{+}$-invariant vector $v \in \mathfrak{f}_{\mid x}$, there exists exactly one $\bar{T}$-invariant cross-section $\nu \in \operatorname{Sec}(\mathfrak{f})$ (determined globally) such that $\nu_{x}=v$.

Proof. A vector bundle $\mathfrak{f}$ over $\mathbb{R}^{n}$ is trivial, therefore we may assume that $\mathfrak{f}=\mathbb{R}^{n} \times V$. $\bar{T}$ determines a homomorphism $T: T \mathbb{R}^{n} \times \mathfrak{g} \rightarrow T \mathbb{R}^{n} \times \operatorname{End}(V)$ such that $\mathcal{L} \circ T=\bar{T} . T$ can be written in the form $T \circ(X, \mu)=T \circ(X, 0)+T \circ(0, \mu)=\left(X, \omega(X)+T^{+} \circ \mu\right)$ $\left(X \in \mathfrak{X}\left(\mathbb{R}^{n}\right), \mu \in \Omega^{0}\left(\mathbb{R}^{n}, \mathfrak{g}\right)\right)$ for a 1 -form $\omega \in \Omega^{1}\left(\mathbb{R}^{n} ; \operatorname{End}(V)\right)$. $\omega$ and $T^{+}$satisfy the following identities (cf. [Ma1, p. 102]):

$$
\begin{gathered}
-d \omega(X, Y)=[\omega(X), \omega(Y)], \\
X\left(T^{+} \circ \mu\right)-T^{+} \circ(X(\mu))+\left[\omega(X), T^{+} \circ \mu\right]=0 .
\end{gathered}
$$

$\nu \in \operatorname{Sec}(\mathfrak{f})$ is $\bar{T}$-invariant if and only if (a) $\mathcal{L}_{T \circ(X, 0)}(\nu)=0,(\mathrm{~b}) \mathcal{L}_{T \circ(0, \mu)}(\nu)=0$. Equation (a) is equivalent to the invariance of $\nu$ with respect to the "reduced representation" $T \mathbb{R}^{n} \hookrightarrow T \mathbb{R}^{n} \times \mathfrak{g} \stackrel{\bar{T}}{\rightarrow} A\left(\mathbb{R}^{n} \times V\right)$ whereas (b) says that, for each $x \in \mathbb{R}^{n}$, the vector $\nu_{x}$ is $\bar{T}_{\mid x}^{+}$-invariant. Condition (a) yields that $0=\mathcal{L}_{(X, \omega(X))}(\nu)=X(\nu)+\mathcal{L}_{\omega(X)}(\nu)$, i.e. the differential equation

$$
X(\nu)=-\mathcal{L}_{\omega(X)}(\nu),
$$

called the differential equation of an invariant cross-section, is satisfied. Taking a basis $w_{1}, \ldots, w_{q}$ of $V$ and writing $\nu=\sum_{i} z^{i} \cdot w_{s}$, we can equivalently replace equation (19.4) by the following system of partial differential equations:

$$
\frac{\partial z^{k}}{\partial x^{i}}=-\sum_{r=1}^{q} a_{i}^{r, k} \cdot z^{r}, \quad i \leq n, l \leq q,
$$


where $a_{i}^{r, k}$ are functions such that $\omega\left(\frac{\partial}{\partial x^{i}}\right)=\sum_{r, k} a_{i}^{r, k} \cdot u_{r, k}, u_{r, k}=w_{r}^{*} \otimes w_{k}$ is a basis of $\operatorname{End}(V)=V^{*} \otimes V$. It is easy to see that equation (19.2) is the condition of the local integrability of the equation (19.5). According to Th. 15.1, the initial conditions $z^{1}(0)=z_{0}^{1}, \ldots, z^{q}(0)=z_{0}^{q}$ (uniquely) determine a solution $\left(z^{1}, \ldots, z^{q}\right)$ of (19.5) defined on the whole $\mathbb{R}^{n}$. It remains to solve the following problem: if the vector $\nu(0)=\sum_{i} z_{0}^{i} \cdot w_{i}$ is $\left(T_{\mid 0}^{+}: \mathfrak{g} \rightarrow \operatorname{End}(V)\right)$-invariant, then, for each $x \in \mathbb{R}^{n}$, the vector $\nu(x)=\sum_{i} z^{i}(x) \cdot w_{i}$ is $T_{\mid x}^{+}$-invariant. The invariance of $\nu(x)$ means that $\mathcal{L}_{T_{\mid x}^{+}(h)}\left(\nu_{x}\right)=0$ for all $h \in \mathfrak{g}$. Therefore it is sufficient to show that the function $\mathcal{L}_{T^{+} \circ \bar{h}}(\nu)$ is identically zero, where $\bar{h}: \mathbb{R}^{n} \rightarrow \mathfrak{g}$ is the constant function $\bar{h}(x)=h$. Put $\beta=\mathcal{L}_{T+\circ \bar{h}}(\nu)$ and assume that $\beta(0)=0$. Using (19.3) and (19.4), we obtain a linear first order differential equation $X(\beta)=\mathcal{L}_{-\omega(X)}(\beta)$ for $X \in \mathfrak{X}\left(\mathbb{R}^{n}\right)$, fulfilled by the function identically equal to zero. On account of the uniqueness of solutions, we have $\beta \equiv 0$, which ends the proof.

As a corollary we obtain

Corollary 19.2. For an arbitrary representation $T: A \rightarrow A(\mathfrak{f})$ of a transitive Lie algebroid $A$ on $\mathfrak{f}$, each invariant cross-section of $\mathfrak{f}$ (defined locally on a connected subset) is uniquely determined by the value at one point. In particular, if such a cross-section is zero at one point, then it is zero globally.

20. Invariant cross-sections over $\mathbb{R} \times M$. Using the previous theorems, we prove that the space of global cross-sections of a vector bundle $f$ over $\mathbb{R} \times M$, invariant with respect to a representation of a regular Lie algebroid $B$ over $(\mathbb{R} \times M, T \mathbb{R} \times E)$, is canonically isomorphic to the space of cross-sections of the vector bundle $\mathfrak{f}_{\mid\left\{t_{0}\right\} \times M}$, invariant with respect to a suitable "restricted" representation. First, we recall the inverse-image of a representation [K2]. Let $A$ be any regular Lie algebroid over $(M, E)$ and $\mathfrak{f}$ any vector bundle on $M$, whereas $f:\left(M^{\prime}, E^{\prime}\right) \rightarrow(M, E)$ any morphism of foliated manifolds. By the inverse-image of a representation $T: A \rightarrow A(\mathfrak{f})$ over $f$ we mean the representation $f^{*} T: f^{\wedge} A \rightarrow A\left(f^{*} \mathfrak{f}\right)$ defined as the superposition $f^{*} T: f^{\wedge} A \stackrel{f^{\wedge} T}{\rightarrow} f^{\wedge}(A(\mathfrak{f})) \stackrel{\mathfrak{c}_{\mathfrak{f}}}{\rightarrow} A\left(f^{*} \mathfrak{f}\right)$ where (a) $f^{\wedge} T$ is a homomorphism of Lie algebroids defined by: $f^{\wedge} T(u, v)=(u, T(v))$, $u \in E^{\prime}, v \in A\left(f_{*} u=\gamma v\right)$, (b) $\mathfrak{c}_{\mathfrak{f}}$ is the canonical strong isomorphism of Lie algebroids such that, for $(u, l) \in f^{\wedge}(A(\mathfrak{f}))_{\mid x}, w:=\mathfrak{c}_{\mathfrak{f}}(u, l)$ has $u$ as its anchor and satisfies the relation: $w(\nu \circ f)=l(\nu)$ for $\nu \in \operatorname{Sec}(\mathfrak{f})$. Obviously, $\mathfrak{c}_{\mathfrak{f}}^{+}$appears as the canonical isomorphism of vector bundles $f^{*}(\operatorname{End}(\mathfrak{f})) \cong \operatorname{End}\left(f^{*} \mathfrak{f}\right)$, and, furthermore, we can write $\left(f^{*} T\right)_{\mid x}^{+}=T_{\mid f(x)}^{+}$ for $x \in M^{\prime}$. We can write $f^{*}\left(g^{*} T\right)=(g \circ f)^{*} T$, see Section 16 .

According to [K2, Ch. 2], the linear mapping $f^{*}: \operatorname{Sec} \mathfrak{f} \rightarrow \operatorname{Sec} f^{*} \mathfrak{f}, \nu \mapsto \nu \circ f$, can be restricted to the space of cross-sections invariant under $T$ and $f^{*} T$, respectively:

$$
f_{I^{0}}^{*}:(\operatorname{Sec} \mathfrak{f})_{I^{0}(T)} \rightarrow\left(\operatorname{Sec} f^{*} \mathfrak{f}\right)_{I^{0}\left(f^{*} T\right)} .
$$

Lemma 20.1. If the saturation of $f\left[M^{\prime}\right]$ with leaves of $E$ equals $M$, then $f_{I^{0}}^{*}$ is a monomorphism.

Proof. Assume that $f_{I^{0}}^{*}(\nu)=0$ for an invariant cross-section $\nu$. This means that $\nu_{f(x)}=0$ for all $x \in M^{\prime}$. Take $x_{0} \in M^{\prime}$ and let $\iota: L \hookrightarrow M$ be the leaf of $E$ passing through $f\left(x_{0}\right), \nu \mid L$ is invariant with respect to $\iota^{*} T: \iota^{\wedge} A \rightarrow A\left(\iota^{*} \mathfrak{f}\right)$. Since $\iota^{\wedge} A$ is transitive Cor. 19.2 yields $\nu \mid L=0$. Saturation of $f\left[M^{\prime}\right]$ with leaves of $E$ implies the equality $\nu \equiv 0$. 
We prove that the space of global cross-sections of a vector bundle $\mathfrak{f}$ over $\mathbb{R} \times M$ invariant with respect to the representation $T: B \rightarrow A(\mathfrak{f})$ of a regular Lie algebroid $B$ over $(\mathbb{R} \times M, T \mathbb{R} \times E)$ is canonically isomorphic to the space of cross-sections of the vector bundle $\mathfrak{f}_{\mid\left\{t_{0}\right\} \times M}$, invariant with respect to a suitable "restricted" representation. The following theorem plays a crucial role in all problems of the homotopy independence of the characteristic homomorphisms considered on the category of Lie algebroids.

THEOREM 20.2 (on invariant cross-sections over $\mathbb{R} \times M$ ). Let $B$ be a regular Lie algebroid over the foliated manifold $(\mathbb{R} \times M, T \mathbb{R} \times E)$ and $\mathfrak{f}$ a vector bundle over $\mathbb{R} \times M$, and $T: A \rightarrow A(\mathfrak{f})$ a given representation. Take $t \in \mathbb{R}$ and the mapping $f_{t}: M \rightarrow \mathbb{R} \times M$, $x \mapsto(t, x)$. Then the restriction mapping $f_{t}^{*}: \operatorname{Sec} \mathfrak{f} \rightarrow \operatorname{Sec} \mathfrak{f}_{\mid\{t\} \times M}\left(\mathfrak{f}_{\mid\{t\} \times M} \cong f_{t}^{*} \mathfrak{f}\right)$ maps isomorphically the space of invariant cross-sections with respect to $T$ onto the space of invariant cross-sections with respect to the restricted representation $f_{t}^{*} T$ :

$$
\left(f_{t}^{*}\right)_{I^{0}}:(\operatorname{Sec} \mathfrak{f})_{I^{0}(T)} \stackrel{\cong}{\rightarrow}\left(\operatorname{Sec} \mathfrak{f}_{\mid\{t\} \times M}\right)_{I^{0}\left(f_{t}^{*} T\right)} .
$$

Proof. Thanks to the Lemma above it remains to show that $\left(f_{t}^{*}\right)_{I^{0}}$ is an epimorphism. Let $\sigma \in \operatorname{Sec}\left(\mathfrak{f}_{\mid\{t\} \times M}\right)$ be an invariant cross-section. Then, for each $x \in M$, the vector $\sigma(x) \in \mathfrak{f}_{\mid(t, x)}$ is invariant with respect to the representation $T_{\mid(t, x)}^{+}: \boldsymbol{g}_{\mid(t, x)} \rightarrow \operatorname{End}\left(\mathfrak{f}_{\mid(t, x)}\right)$. Consider the embedding $f_{x}: \mathbb{R} \rightarrow \mathbb{R} \times M, t \mapsto(t, x)$. Since $\operatorname{Im}\left(f_{x}\right)=\mathbb{R} \times\{x\}$ is contained in some leaf of $T \mathbb{R} \times E$, therefore $\left(f_{x}\right)^{\wedge}(B)$ is a transitive and, by Cor. 18.3, trivial Lie algebroid. Th. 19.1 (also Th. IV.1.19 of [Ma1, p.195]) yields that the vector $\sigma(x)$ can be uniquely extended to some $C^{\infty}$ cross-section $\sigma_{x}$ of the vector bundle $\left(f_{x}\right)^{*}(\mathfrak{f})=\mathfrak{f}_{\mid \mathbb{R} \times\{x\}}$, invariant with respect to the representation $\left(f_{x}\right)^{*} T:\left(f_{x}\right)^{\wedge}(B) \rightarrow A\left(\mathfrak{f}_{\mid \mathbb{R} \times\{x\}}\right)$. The family $\left\{\sigma_{x}: x \in M\right\}$ determines a global cross-section $\sigma^{1}: \mathbb{R} \times M \rightarrow \mathfrak{f}$ by the formula: $\sigma^{1}(t, x)=$ $\sigma_{x}(t)$. It is evident that $\left(f_{t}\right)^{*}\left(\sigma^{1}\right)=\sigma$. To end the proof, all we need is to show (a) the smoothness of $\sigma^{1}$, (b) the $T$-invariance of $\sigma^{1}$. First, we check (a). For this purpose, take $x_{0} \in M$ and a simple distinguished open neighbourhood $U \subset M$ of $x_{0}$ [the domain of some distinguished chart of the foliation $E]$. The foliation $E_{\mid U}$ has a global connected transversal manifold, say $N$, and its leaves are diffeomorphic to a Euclidean space. Then $N^{\prime}:=\{t\} \times N$ is a transversal manifold of the distribution $T \mathbb{R} \times E$. The cross-section

$$
\sigma_{0}: N^{\prime} \rightarrow \mathfrak{f}_{\mid N^{\prime}}, \quad(t, x) \mapsto \sigma(x),
$$

is $C^{\infty}$ and invariant with respect to the representation $j^{*} T, j: N^{\prime} \hookrightarrow M^{\prime}:=\mathbb{R} \times U \subset$ $\mathbb{R} \times M$ being the inclusion, moreover, $\sigma^{\prime}:=\sigma^{1} \mid M^{\prime}$ is some extension of $\sigma_{0}$.

Let $B^{\prime}:=B_{\mid M^{\prime}} . B^{\prime}$ is a regular Lie algebroid over $\left(\mathbb{R} \times U, T \mathbb{R} \times\left(E_{\mid U}\right)\right)$. Leaves of the foliation $T \mathbb{R} \times\left(E_{\mid U}\right)$ are of the form $\mathbb{R} \times L$ where $L$ is a leaf of $E_{\mid U}$. They are diffeomorphic to a Euclidean space and proper; $N^{\prime}$ is a global transversal manifold of $T \mathbb{R} \times\left(E_{\mid U}\right)$ which cuts each leaf in exactly one point. Therefore, it is obvious that, without loss of generality, we may assume that

$-M^{\prime}=\mathbb{R}^{p} \times \mathbb{R}^{q}$,

- $B^{\prime}$ is over $\left(\mathbb{R}^{p} \times \mathbb{R}^{q}, T \mathbb{R}^{p} \times 0\right)$.

In the context considered above, $p$ is equal to the dimension of the foliation $T \mathbb{R} \times E$ and $q=\operatorname{dim} N^{\prime}$. By the proof of Th. 17.1, we obtain the existence of global crosssections $Y_{1}, \ldots, Y_{p} \in \operatorname{Sec} B^{\prime}$ such that $\gamma \circ Y_{i}=\frac{\partial}{\partial y^{i}}$ and $\llbracket Y_{i}, Y_{j} \rrbracket=0, i, j \leq p$. Moreover, 
in the remainder of the proof of our theorem, we can assume that $N^{\prime}=\{0\} \times \mathbb{R}^{q}$, $\mathfrak{f}=\left(\mathbb{R}^{p} \times \mathbb{R}^{q}\right) \times V$ and $A(\mathfrak{f})=T\left(\mathbb{R}^{p} \times \mathbb{R}^{q}\right) \times \operatorname{End}(V)$. In this context, a $C^{\infty}$ cross-section $\sigma_{0}:\{0\} \times \mathbb{R}^{q} \rightarrow \mathfrak{f}_{\mid\{0\} \times \mathbb{R}^{q}}$ such that $\sigma_{0}(0, y)$ is invariant with respect to the representation $T_{\mid(0, y)}^{+}: \boldsymbol{g}_{\mid(0, y)} \rightarrow \operatorname{End}(V)$ is given, and we know that there exists a cross-section $\sigma^{\prime}:$ $\mathbb{R}^{p} \times \mathbb{R}^{q} \rightarrow \mathfrak{f}$ (whose smoothness we are proving) extending $\sigma_{0}$ and such that $\sigma^{\prime} \mid \mathbb{R}^{p} \times\left\{y^{0}\right\}$ is, for each $y^{0} \in \mathbb{R}^{q}$, of class $C^{\infty}$ and invariant with respect to the representation $T_{\mid \mathbb{R}^{p} \times\left\{y^{0}\right\}}$ of the transitive Lie algebroid $B_{\mid \mathbb{R}^{p} \times\left\{y^{o}\right\}}^{\prime}$ on $\mathfrak{f}_{\mid \mathbb{R}^{p} \times\left\{y^{o}\right\}}$ (the restriction $\ldots \mid \mathbb{R}^{p} \times\left\{y^{o}\right\}$ is understood as the inverse-image over the suitable inclusion).

Let $T \circ Y_{i}=\left(\frac{\partial}{\partial y^{i}}, c^{i}\right)$ for some $c^{i}: \mathbb{R}^{p} \times \mathbb{R}^{q} \rightarrow \operatorname{End}(V), i \leq p$. The fact that $T$ is a representation gives the equality: $0=T \llbracket Y_{i}, Y_{j} \rrbracket=\left(0, \frac{\partial c_{j}}{\partial y^{i}}-\frac{\partial c_{i}}{\partial y^{j}}+\left[c_{i}, c_{j}\right]\right)$, i.e.

$$
\left[c_{i}, c_{j}\right]=\frac{\partial c_{i}}{\partial y^{j}}-\frac{\partial c_{j}}{\partial y^{i}}, \quad i, j \leq p .
$$

Let $w_{1}, \ldots, w_{n}$ be a basis of $V$; write $c_{i}(x)\left(w_{s}\right)=\sum_{k=1}^{n} c_{s i}^{k}(x) \cdot w_{k}, x \in \mathbb{R}^{p} \times \mathbb{R}^{q}$. It follows immediately that (20.1) is equivalent to the following conditions:

$$
\frac{\partial c_{r i}^{k}}{\partial y^{s}}-\frac{\partial c_{r s}^{k}}{\partial y^{i}}=\sum_{u=1}^{n} c_{r i}^{u} \cdot c_{u s}^{k}-\sum_{u=1}^{n} c_{r s}^{u} \cdot c_{u i}^{k}, \quad i, s \leq p, k, r \leq n .
$$

The invariance of a cross-section $\tau \in \operatorname{Sec} \mathfrak{f}=\Omega^{0}\left(\mathbb{R}^{p} \times \mathbb{R}^{q}, V\right)$ with respect to the representation $T: B^{\prime} \rightarrow T\left(\mathbb{R}^{p} \times R^{q}\right) \times \operatorname{End}(V)$ means that $\mathcal{L}_{T \circ X}(\tau)=0$ for all $X \in \operatorname{Sec}\left(B^{\prime}\right)$, in particular, that $\mathcal{L}_{T \circ Y_{i}}(\tau)=0, i \leq p$. The last condition says that $\frac{\partial \tau}{\partial y^{i}}+c_{i}(\tau)=0$, $i \leq p$, or, equivalently,

$$
\frac{\partial \tau^{k}}{\partial y^{i}}=-\sum_{r=1}^{n} c_{r i}^{k} \cdot \tau^{r}, \quad i \leq p .
$$

System $(20.3)$ of differential equations is of the first order in the parameters $\left(y^{p+1}, \ldots\right)$. It is easy to notice that (20.2) forms conditions of the local integrability of (20.3). From Th. 15.1 it follows that there exists exactly one (globally defined) $C^{\infty}$ cross-section $\tilde{\sigma}$ : $\mathbb{R}^{p} \times \mathbb{R}^{q} \rightarrow \mathfrak{f}$ which is a solution of (20.3) and satisfies the given initial condition $\tilde{\sigma}(0, y)=$ $\sigma_{0}(0, y), y \in \mathbb{R}^{q}$. Of course, $\tilde{\sigma}=\sigma^{\prime}$, which confirms the smoothness of $\sigma^{\prime}$. Finally, we see that (b) now follows trivially.

\section{References}

[An] G. Andrzejczak, Some characteristic invariants of foliated bundles, Dissert. Math. 222 (1984).

[At] M. Atiyah, Complex analytic connections in fibre bundles, Trans. Amer. Math. Soc. 85 (1957), 181-207.

[A-M] R. Almeida and P. Molino, Suites d'Atiyah et feuilletages transversalement complets, C. R. Acad. Sci. Paris Sér. I Math. 300 (1985), 13-15.

[B-K-W] B. Balcerzak, J. Kubarski and W. Walas, Primary characteristic homomorphism of pairs of Lie algebroids and Mackenzie algebroid, this volume.

[B1] I. W. Belko, Characteristic classes of transitive Lie algebroids, preprint Minsk, 1994, 192 pp. (in Russian). 
[B2] -, Characteristic homomorphism of a Lie algebroid, News Belarus Acad. Sci. Ser. phys.-math. sci. 1, 1997 (in Russian).

[C-D-W] A. Coste, P. Dazord and A. Weinstein, Groupoïdes symplectiques, Publ. Dep. Math. Univ. Lyon 1, 2/A (1987).

[Ca] H. Cartan, Cohomologie réelle d'un espace fibré principal différentiable, Séminaire Cartan, exposés 19 et $20(1949 / 50)$.

[C-S1] S. S. Chern and J. Simons, Some cohomology classes in principal fibre bundles and their applications to Riemannian geometry, Proc. Nat. Acad. Sc. USA 68 (1971), 791-794.

[C-S2] - - Characteristic forms and geometric invariants, Annals of Math. 99 (1974), 48-69.

[Cr] M. Crainic, Differentiable and algebroid cohomology, Van Est isomorphisms, and characteristic classes, preprint, arXiv:math.DG/0008064.

[D] P. Dazord, electronic letter, 1994.

[E] C. Ehresmann, Les connexions infinitésimales dans un espace fibré différentiable, in: Colloque de topologie (espaces fibrés), Bruxelles, 1950, 29-55, Liège 1951.

[F1] R. L. Fernandes, Connections in Poisson geometry I: Holonomy and Invariants, J. Differential Geometry 54 (2000), 303-366.

[F2] - Lie algebroids, holonomy and characteristic classes, preprint DG/007132.

[G] W. Greub, Multilinear Algebra, Springer, 1967.

[G-H-V] W. Greub, S. Halperin and R. Vanstone, Connections, Curvature, and Cohomology, Vol. II, Lie groups, principal bundles, and characteristic classes, Acad. Press, 1973.

[H] J.-C. Herz, Pseudo-algèbres de Lie, C. R. Acad. Sci. Paris 236 (1953), I, 1935-1937 and II, 2289-2291.

[H-M] P. Higgins and K. Mackenzie, Algebraic constructions in the category of Lie algebroids, J. Algebra 129 (1990), 194-230.

[H1] J. Huebschmann, Poisson cohomology and quantization, J. Reine Angew. Math. 408 (1990), 57-113,

[H2] - Extensions of Lie-Rinehart algebras and the Chern-Weil homomorphism, in: Contemp. Math. 227, 1999.

[K-T1] F. Kamber and P. Tondeur, Characteristic invariants of foliated bundles, Manuscripta Math. 11 (1974), 52-89.

[K-T2] - Foliated Bundles and Characteristic Classes, Lecture Notes in Math. 493, Springer, 1975.

[K-SB] Y. Kerbrat and Z. Souici-Benhammadi, Variétés de Jacobi et groupoïdes de contact, C. R. Acad. Sci. Paris 317 (1993), 81-86.

[K1] J. Kubarski, Lie algebroid of a principal fibre bundle, Publ. Dep. Math. Univ. Lyon 1, 1/A 1989.

[K2] -, The Chern-Weil homomorphism of regular Lie algebroids, Publ. Dep. Math. Univ. Lyon 1, 1991. (http://im0.p.lodz.pl/ ${ }^{\sim}$ kubarski/forum)

[K3] - A criterion for the minimal closedness of the Lie subalgebra corresponding to a connected nonclosed Lie subgroup, Rev. Mat. Univ. Complut. Madrid 4 (1991).

[K4] - Characteristic classes of regular Lie algebroids - a sketch, in: Proceedings of the Winter School Geometry and Physics (Srni, 1991), Suppl. Rend. Circ. Mat. Palermo 30 (1993).

[K5] - Tangential Chern-Weil homomorphism, in Geometric Study of Foliations (Tokyo, 1993), ed. by T. Mizutani, World Sci., 1994, 327-344. 
[K6] -, Bott's vanishing theorem for regular Lie algebroids, Trans. Amer. Math. Soc. 348 (1996).

[K7] - Algebroid nature of the characteristic classes of flat bundles, in: Homotopy and Geometry, Banach Center Publ. 45, Inst. Math., Polish Acad. Sci., Warszawa, 1998, 199-224.

[K8] - Fibre integral in regular Lie algebroids, in: New Developments in Differential Geometry (Budapest, 1996), Kluwer, 1999.

[K9] - Poincaré duality for transitive unimodular invariantly oriented Lie algebroids, Topology Appl., in print.

[K10] - Gysin sequence and Euler class of spherical Lie algebroids, Publ. Math. Debrecen, in print.

[L] P. Libermann, Sur les prolongements des fibrés principaux et les groupoïdes différentiables, Séminaire Analyse Global, Montréal, 1969.

[Ma1] K. Mackenzie, Lie Groupoids and Lie Algebroids in Differential Geometry, London Math. Soc. Lecture Note Ser. 124, Cambridge, 1987.

[Ma2] - Lie algebroids and Lie pseudoalgebras, Bull. London Math. Soc. 27 (1995), 97-147.

[M-Sch] C. C. Moore and C. Schochet, Global Analysis on Foliated Spaces, Math. Sci. Res. Inst. Publ. 9, Springer, 1988.

[Mo1] P. Molino, Étude des feuilletages transversalement complets et applications, Ann. Sci. Ecole Norm. Sup. 10 (1977), 289-307.

[Mo2] —, Riemannian Foliations, Progr. Math. 73, Birkhäuser, 1988.

[S] A. M. Silva, Atiyah sequence and complete closed pseudogroups preserving a local parallelism, in: Holomorphic Dynamics (Mexico City, 1986), Lecture Notes in Math. 1345 (1988), 302-316.

[T1] N. Teleman, Cohomology of Lie algebras, in: Global Analysis and its Applications Vol. III (Trieste, 1972), Internat. Atomic Energy Agency, Vienna, 1974, 195-202.

[T2] -, A characteristic ring of a Lie algebra extension, Acad. Naz. Lincei Rend. Cl. Sci. Fis. Mat. Natur. (8) 52 (1972), 498-506 and 708-711.

[NVQ] Ngo-Van-Que, Du prolongement des espaces fibrés et des structures infinitésimales, Ann. Inst. Fourier (Grenoble) 17,1 (1967), 157-223. 